Review

\title{
Plasticity and geophysical flows: A review
}

\author{
Christophe Ancey* \\ Ecole Polytechnique Fédérale de Lausanne, Lausanne, Switzerland
}

Received 18 February 2006; received in revised form 26 April 2006; accepted 5 May 2006

\begin{abstract}
The objective of this review is to examine how the concept of plasticity is used in geophysical fluid dynamics. Rapid mass movements such as snow avalanches or debris flows involve slurries of solid particles (ice, boulder, clay, etc.) within an interstitial fluid (air, water). The bulk behavior of these materials has often been modeled as plastic materials, i.e., a plastic material yields and starts to flow once its stress state has significantly departed from equilibrium. Two plastic theories are of common use in fluid dynamics: Coulomb plasticity and viscoplasticity. These theories have little in common, since ideal Coulomb materials are two-phase materials for which pore pressure and friction play the key role in the bulk dynamics, whereas viscoplastic materials (e.g., Bingham fluids) typically behave as single-phase fluids on the macroscopic scale and exhibit a viscous behavior after yielding. Determining the rheological behavior of geophysical materials remains difficult because they encompass coarse, irregular particles over a very wide range of size. Consequently, the true nature of plastic behavior for geophysical flows is still vigorously debated. In this review, we first set out the continuum-mechanics principles used for describing plastic behavior. The notion of yield surface rather than yield stress is emphasized in order to better understand how tensorial constitutive equations can be derived from experimental data. The notion of single-phase or two-phase behaviors on the macroscopic scale is then examined using a microstructural analysis on idealized suspensions of spheres within a Newtonian fluid; for these suspensions, the single-phase approximation is valid only at very high or low Stokes numbers. Within this framework, the bulk stress tensor can also be constructed, which makes it possible to give a physical interpretation to yield stress. Most of the time, depending on the bulk properties (especially, particle size) and flow features, bulk behavior is either Coulomb-like or viscoplastic in simple-shear experiments. The consequences of the rheological properties on the flow features are also examined. Some remarkable properties of the governing equations describing thin layers flowing down inclined surfaces are discussed. Finally, the question of parameter fitting is tackled: since rheological properties cannot be measured directly in most cases, they must be evaluated from field data. As an example, we show that the Coulomb model successfully captures the main traits of avalanche motion, but statistical analysis demonstrates that the probability distribution of the friction coefficient is not universal.
\end{abstract}

(C) 2006 Elsevier B.V. All rights reserved.

Keywords: Plasticity; Yield stress; Avalanche; Debris flow; Creeping flow; Shallow-flow equations

\section{Contents}

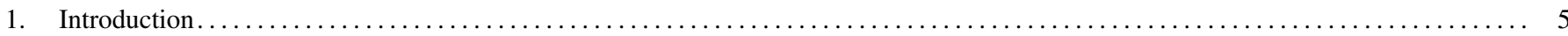

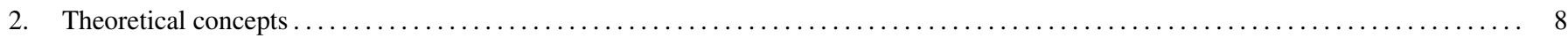

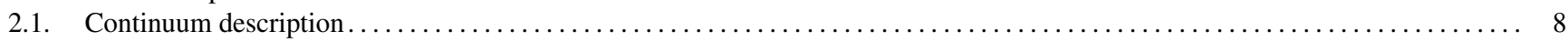

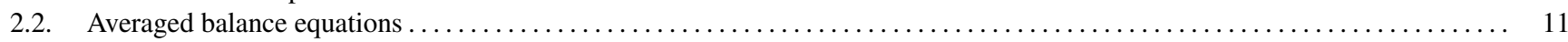

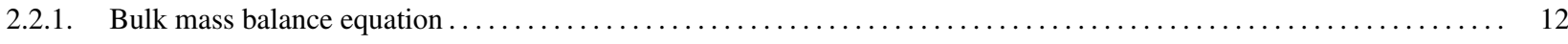

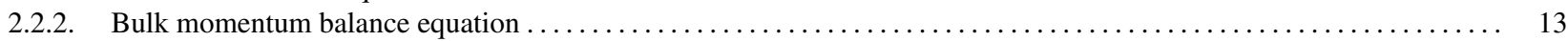

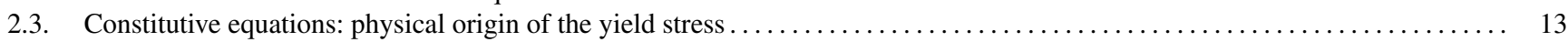

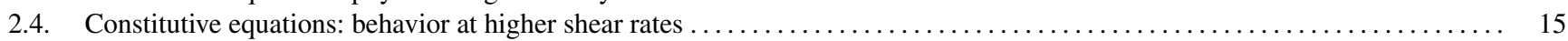

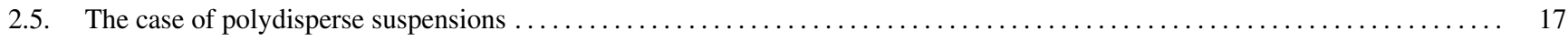

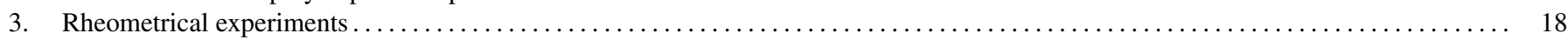

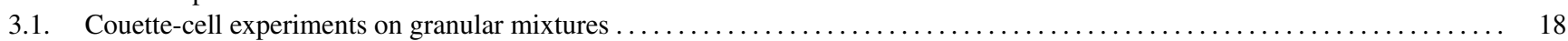

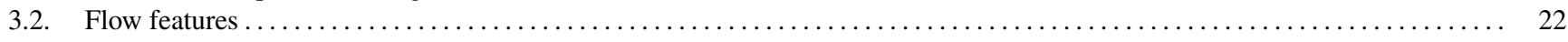

\footnotetext{
* Tel.: +41 2169311 11/32 87; fax: +41216936767.

E-mail address: christophe.ancey@epfl.ch.
} 


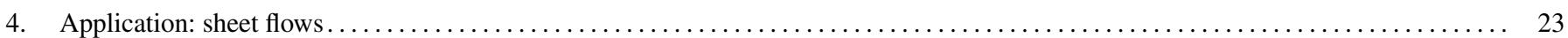

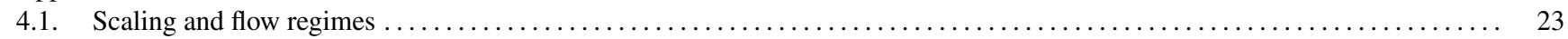

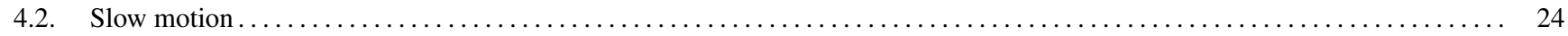

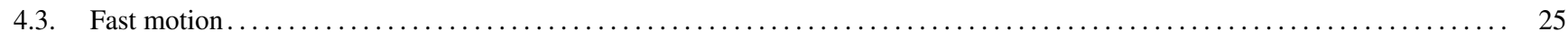

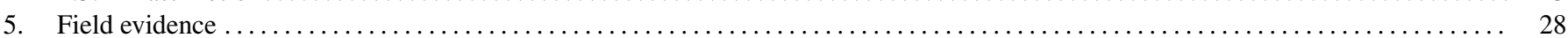

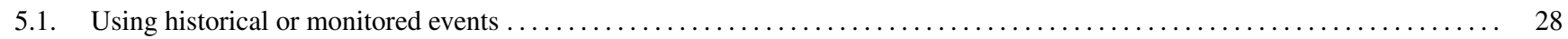

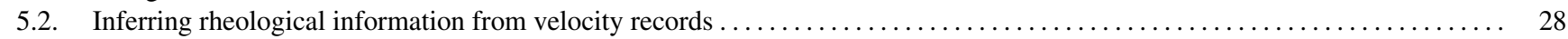

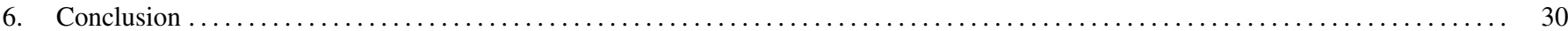

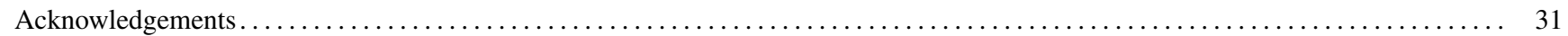

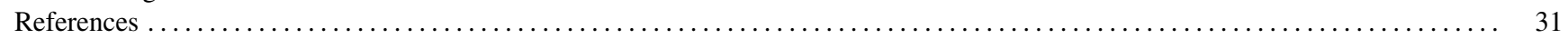

\begin{tabular}{|c|c|}
\hline \multicolumn{2}{|c|}{ Nomenclature } \\
\hline$a$ & particle radius \\
\hline$C$ & torque \\
\hline d & tensor of strain rate \\
\hline$D_{2}$ & second invariant of the strain-rate tensor \\
\hline$f$ & friction coefficient \\
\hline$f$ & yield function \\
\hline$g$ & gravity acceleration \\
\hline$h$ & flow depth \\
\hline$h_{0}$ & position of the yield surface \\
\hline$H$ & characteristic function \\
\hline$I_{1}$ & first invariant of the stress tensor \\
\hline$I_{2}$ & second invariant of the stress tensor \\
\hline$J_{2}$ & second invariant of the strain-rate tensor \\
\hline $\mathbf{k}$ & unit normal \\
\hline$M$ & reduced torque \\
\hline & particle mass \\
\hline$n$ & density number \\
\hline$p$ & pressure \\
\hline$P_{*}$ & pressure scale \\
\hline$R e$ & Reynolds number \\
\hline$R e_{\mathrm{p}}$ & particle Reynolds number \\
\hline & radius of the inner cylinder \\
\hline $\mathbf{s}$ & extra-stress tensor (or deviatoric stress tensor) \\
\hline$s$ & distance between the surface of two particles \\
\hline$S t$ & Stokes number \\
\hline$S$ & dimensionless shear stress \\
\hline$t$ & time \\
\hline$T$ & temperature \\
\hline $\mathbf{u}$ & velocity \\
\hline $\mathbf{u}^{\prime}$ & velocity fluctuation \\
\hline & fluid velocity \\
\hline & particle velocity \\
\hline & velocity scale \\
\hline $\mathcal{V}$ & volume of control \\
\hline \multicolumn{2}{|c|}{ Greek letters } \\
\hline$\delta$ & phase (Lode's) angle \\
\hline$\epsilon$ & depth-to-height ratio \\
\hline$\eta$ & bulk viscosity \\
\hline$\dot{\gamma}$ & shear rate \\
\hline
\end{tabular}

\begin{tabular}{|ll|}
\hline & \\
$\kappa$ & dimensionless rotational velocity \\
$\mu$ & coefficient of permeability \\
$\Omega$ & dynamic viscosity \\
$\phi$ & rotational velocity of the inner cylinder (Couette) \\
$\phi_{\mathrm{c}}$ & critical solid concentration (percolating network) \\
$\phi_{\mathrm{f}}$ & solid concentration in fine (colloidal) particles \\
$\phi_{\mathrm{m}}$ & maximum solid concentration \\
$\phi_{\mathrm{t}}$ & total solid concentration (for bimodal mixtures) \\
$\Psi$ & particle energy potential \\
$\rho$ & density \\
$\rho_{\mathrm{f}}$ & fluid density \\
$\rho_{\mathrm{p}}$ & particle density \\
$\bar{\rho}$ & bulk density \\
$\bar{\rho}^{\prime}$ & buoyant bulk density \\
$\sigma$ & extra-stress tensor \\
$\sigma^{(\mathrm{f})}$ & solid contribution to the fluid stress tensor \\
$\sigma^{(\mathrm{p})}$ & solid contribution to the bulk stress tensor \\
$\sigma_{\mathrm{f}}$ & extra-stress tensor for a fluid phase \\
$\sigma_{\mathrm{p}}$ & extra-stress tensor for a solid particle \\
$\sigma$ & normal stress \\
$\sigma^{\prime}$ & effective normal stress \\
$\tau$ & shear stress \\
$\tau_{\mathrm{b}}$ & bottom shear stress \\
$\tau_{\mathrm{c}}$ & yield stress \\
$\varphi$ & bulk friction angle \\
$\zeta$ & similarity variable \\
& \\
& \\
\hline & \\
\hline & \\
\hline & \\
\hline &
\end{tabular}

\section{Introduction}

A number of geophysical flows involve rapid gravity-driven mass movements of solid particles within a fluid. Typical examples include snow avalanches (Fig. 1) [5], debris flows (Fig. 2) [129], lava flows (Fig. 3) [103], and submarine avalanches $[88,105]$. These flows usually take the appearance of viscous fluids flowing down a slope and this observation has prompted the use of fluid-mechanics tools for describing their motion. However, the impediments to a full fluid-mechanics approach are many: a wide range of particle size (often in the $10^{-6}$ to $1 \mathrm{~m}$ range), composition that may change with time and/or position, ill-known boundary conditions (e.g., erodible basal sur- 


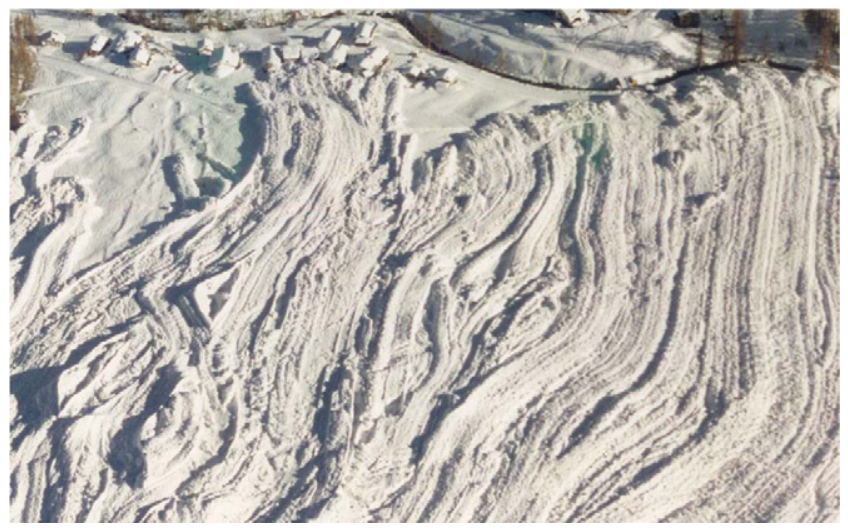

Fig. 1. Huge avalanche deposit in the Tarentaise valley (France). The chalets at the top left corner gives a scale. The deposit thickness was approximately $10 \mathrm{~m}$ in the runout zone. The deposit width was approximately $300 \mathrm{~m}$. This avalanche occurred in March 1995 after heavy snowfalls (Courtesy of M. Margot, Peloton de Gendarmerie de Bourg-Saint-Maurice).

face) and initial conditions, time-dependent flows with abrupt changes (e.g., surge front, instabilities along the free surface), etc. Even with the construction of specifically devoted large rheometers $[62,67,158,190]$, testing the rheometrical properties of samples collected in the field remains difficult. To give examples of materials involved in rapid mass movements, Fig. 4 reports different types of snow observed in avalanche deposits. Because of particle size and thermodynamic alteration (snow is highly sensitive to changes in air temperature), using classic rheometers with these materials does not make sense. All these difficulties pose great challenges in any fluid-mechanics approach for modeling rapid mass movements and have given impetus to extensive research combining laboratory and field experiments, theory, field observation, and numerical simulations $[120,126]$.

The idea of plastic behavior appeared very early in the engineering literature to characterize the rheological behavior of materials involved in rapid mass movements [132,174]. At the very beginning, this idea was used to explain why bulk materials behave like solids when they are at rest and why, under

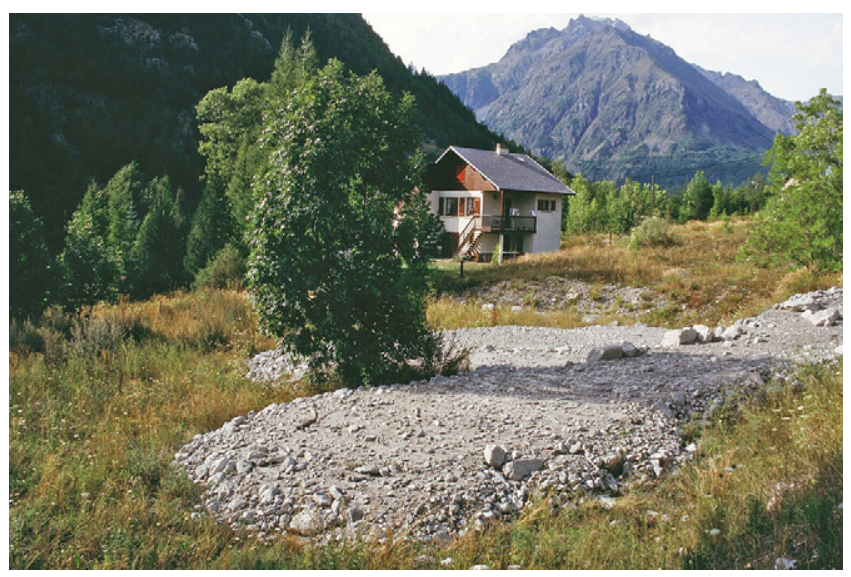

Fig. 2. Deposit of a debris flow on an alluvial fan in the Pelvoux valley (France). The house in the background gives an indication of scale. The deposit thickness was approximately $30 \mathrm{~cm}$. Photograph taken by the author in Pelvoux (France) in July 1998.

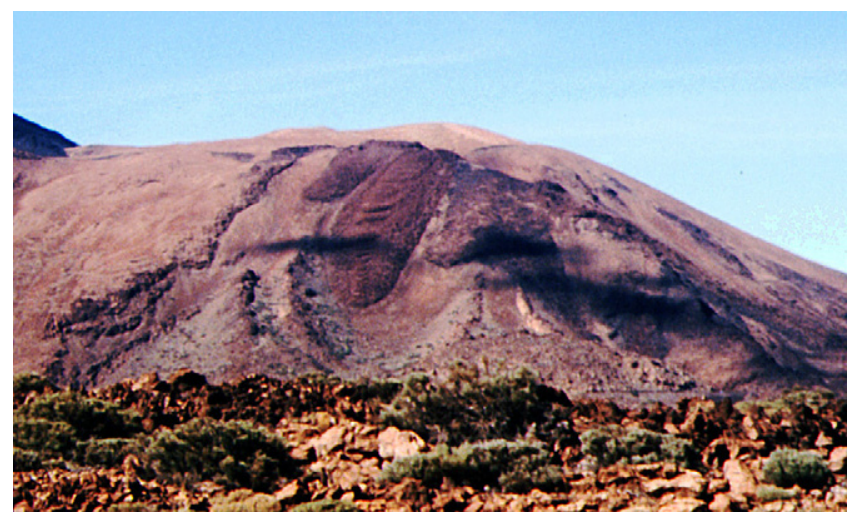

Fig. 3. Lava deposit Montaña Blanca, Teide Peak, Tenerife. The deposit width and length were approximately 50 and $100 \mathrm{~m}$, respectively. Courtesy of Dr. E. Bardou, University of Lausanne.

some circumstances, they yield and start to flow like fluids. In the meantime, over the last century, plastic behavior was extensively studied for a wide range of materials including metals, alloys, concrete, soils, ice, rocks, fiber composites, and many other brittle materials [48,110,169,201,234].

In soil mechanics, soils and geomaterials are most often satisfactorily modeled within the framework of elastoplasticity with a strain softening/hardening yield surface and the non-associate flow rule. Research on plastic behavior takes its roots in the pioneering work of Coulomb [63], who described the stability of piles and embankments, and in the seminal paper of Mohr [173], which contained the fundamentals of stress analysis. Since then, both experimental and theoretical results have helped clarify the notion of plasticity by distinguishing elastic limit, irreversible deformation (i.e., plastic deformation), dilatancy, yielding (critical state), shear localization, and post-failure behavior [76,86,204,208,217,234]. Specific analytical tools based on incremental mechanics and micromechanics analysis have been used to explain the relationships between bulk and particle behaviors $[60,176,234]$.

In the rheology of concentrated suspensions, the notion of plasticity is far less consensual. Historically, this notion is intimately associated with the name of Bingham, who not only coined the word "rheology", but also proposed the first empirical law including a yield stress in 1922: the Bingham law [43]. Contrary to solid mechanicians, for whom plasticity means loss of reversibility in material deformations, fluid rheologists have related plasticity to solid/fluid transition: the yield stress of a solid would be the stress at which the solid first starts to deform continuously, i.e. to flow. In this perspective, the yield stress marks the limit between solid-like (assumed to be elastic) and fluid-like (viscous) behaviors. The review by Bird et al. [44] has documented a large number of everyday-life materials that belong to the family of viscoplastic fluids: food products (e.g., ice cream, mayonnaise), blood, industrial slurries, household consumer products (e.g., lotions, spreads), etc. At the very beginning, defining the yield stress as the threshold for incipient motion was widely accepted. A consistent tensorial formulation of the phenomenological Bingham law was proposed by Prager $[115,196]$ and Oldroyd $[183]$ in a way that was very close to the 


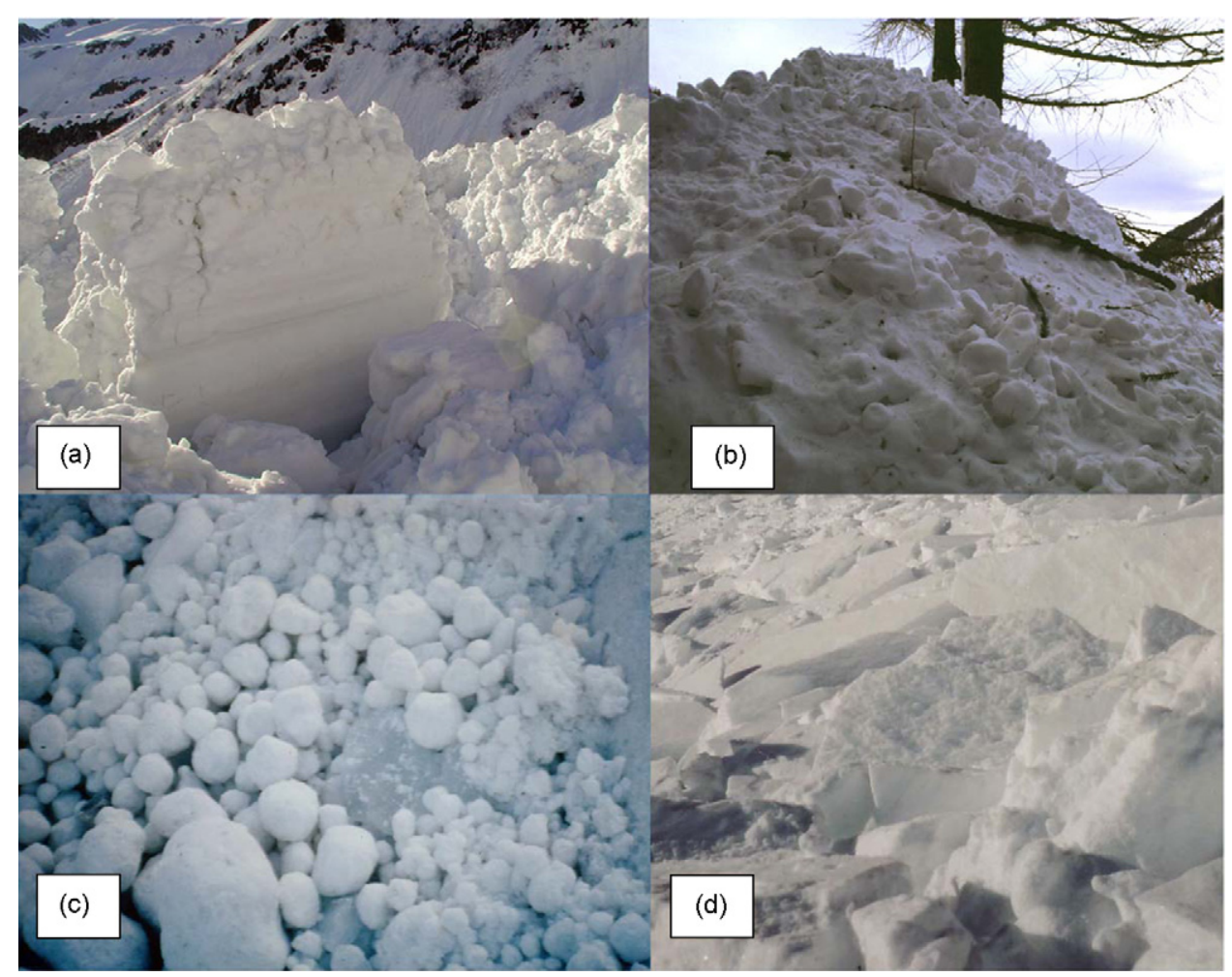

Fig. 4. Different types of snow observed in avalanche deposits. (a) Block of wet snow (size: $1 \mathrm{~m}$ ). (b) Slurry of dry snow including weak snowballs formed during the course of the avalanche (the heap height was approximately $2 \mathrm{~m}$ ). (c) Ice balls involved in a huge avalanche coming from the North face of the Mont Blanc (France); the typical diameter was $10 \mathrm{~cm}$. (d) Sintered snow forming broken slabs (typical length: $40 \mathrm{~cm}$, typical thickness $10 \mathrm{~cm}$ ).

treatment done for other continua at the same time (before the 1950s). After the 1950s, the concept of plasticity evolved differently in solid and fluid mechanics. While new concepts such as nonassociate flow rule or hardening emerged in solid mechanics over the period from 1950 to 1970 , nothing seemed to modify the belief of fluid rheologists in the yield-stress concept until 1985, when Barnes and Walters stated in a provocative paper [31] that the yield stress does not exist. This paper was the starting point of a long and intense debate within the fluid rheology community on the meaning of yield stress, with two tentative conclusions:

- For low shear rates, evidence has been accumulating, showing that yielding and thixotropy are tightly interconnected $[28,71,72,74]$. Indeed, yielding is associated with several complex processes such as changes in the particle arrangement and/or the dynamics of particle contact, which are history-dependent processes. Several phenomenological models have been proposed to describe yielding in a viscoplastic fluid; most constitutive models include a kinetic equation governing the variations in a structural parameter (i.e., describing particle arrangement within the suspension) [41,192].

- With increasing shear rates, the processes involved in the material yielding are of decreasing importance. It is then possible to define an apparent yield stress as the intersect of the flow curve and the axis $\dot{\gamma}=0$. Although this extrapolated yield stress differs from the values measured in quasi-static conditions $[58,59]$, it can be easily and robustly evaluated, which impelled some authors to state that yield stress was an engineering reality $[18,49,106]$. Several empirical laws including the Bingham, Casson, and Herschel-Bulkley are commonly used to describe the flow curve [44].

It is worth noting that in fluid rheology, the question of plasticity boils down to the definition of yield stress and most viscoplastic laws are given in a scalar form and are valid for simpleshear flows. Since Prager's and Oldroy's derivation, little work has been done on the tensorial formulation of constitutive equations for viscoplastic materials and the notion of yield surface (i.e., the three-dimensional generalization of the yield stress in a stress space) [96]. To a large extent, this can be explained by how difficult it is with fluids to properly run experiments in flow geometries other than simple-shear flows. The other reason is that it was not until the 1980s that it was possible to carry out experiments at very low shear rates and thus to explore the solid/fluid transition [29].

In geophysical fluid mechanics, there have been many attempts to describe the rheological behavior of natural materials $[22,125]$. However, since rheometrical experiments are no way easy, scientists have to use proxy procedures to characterize the rheological behavior of natural materials. Interpreting the traces of past events (e.g., shape of deposits), running small-scale experiments with materials mimicking the behavior of natural materials, and making analogies with idealized materials are common approaches to this issue. Because of a lack of experimental validation, there are many points of contention within the different communities working on geophysical flows. 
A typical example is provided by the debate around the most appropriate constitutive equation for describing sediment mixtures mobilized by debris flows [127]: a certain part of the debrisflow community uses soil-mechanics concepts (Coulomb behavior) $[128,129]$, while another part prefers viscoplastic models [65]. A third category merges the different concepts from soil and fluid mechanics to provide general constitutive equations [32,55-57,179,180,227].

The objective of this review is to examine how the concept of plasticity is used in geophysical fluid mechanics. In the applications, we will focus on rapid gravity-driven mass movements such as avalanches and debris flows. We will first present the theoretical concepts underpinning plasticity theory in Section 2. In Section 2.1, we will explain, at a basic level, how a plastic flow rule is built using the principles of continuum mechanics. Emphasis will be placed on the differences between Coulomb plasticity and viscoplasticity concerning not only the yield surface, but also the one-phase/two-phase character of flows associated with a plastic behavior. After this description of plasticity on the bulk scale, we will examine the physical origins of plasticity by examining what happens on the particle scale. In Section 2.2, we will start by deriving the equations of motion for an idealized suspension made up of spherical equal-size particles in a Newtonian fluid. We will explain how the bulk stress tensor can be defined from the particle behavior. In Section 2.3, we will provide a physical interpretation of yield stress for colloidal and noncolloidal systems, while in Section 2.4 we will derive the constitutive equations depending on the flow regime. In Section 2.5 , we will outline the problems related to particle suspensions when the particle-size range is very wide, which is the usual case for geophysical flows.

In Section 3, we will overview experiments focusing on the rheological determination of natural materials. A critical point compared to model suspensions is that the diversity of particle sizes and types gives rise to odd behaviors. We will especially address the key issue of viscoplastic transition: when we increase the fine-fraction content in a coarse-grained suspension, we observe a radical change in behavior, marked by a transition from a Coulomb frictional regime to a viscoplastic regime. Both rheometrical and flume experiments provide evidence for this transition. We will also show that for poorly sorted materials, the rheological properties are strongly time-dependent and, depending on the typical flow timescale, the bulk can exhibit either Coulomb-like or viscoplastic properties.

In Section 4, we will examine how rheological information can be used to derive the equations of motion for free-surface, gravity-driven flows. As the governing equations express the balance between inertia, pressure gradient, and friction, different regimes may be achieved depending on the relative strength of each process relative to the others. Attention is paid to slow flows, for which the pressure gradient is balanced by viscous dissipation. In this case, analytical asymptotic solutions can be derived. We will also spend some time on fast flows, for which there is no dominant term in the governing equations. In that case, the Saint-Venant approach (i.e., depth-averaging the equations of motion) can be used to simplify the equations of motion. Analytical solutions will be derived for some flow geometries (i.e., the so-called dam-break problem and kinematic-wave approximation).

Finally, in Section 5, we will focus our attention on field evidence. Interpreting the deposits of geophysical flows may sometimes be instructive. A very common procedure is to assume the form of the constitutive equation and then to adjust the rheological parameters by matching certain flow features (e.g., the run-out distance) and field measurements. On rare occasions, using velocity measurements makes it possible to derive rheological information. Statistical analysis can be used to check the robustness of parameter fitting.

The reader can also refer to other review papers dealing with constitutive equations and geophysical flows. In the context of geophysical flows, Savage studied granular flows [211]. Hutter et al. realized that most constitutive equations used for debris flows were given in a scalar form (simple-shear flow). These authors provided a unified framework based on continuum mechanics in order to classify and generalize the existing phenomenological laws [125]. More recently, Dartevelle reviewed the processes and related constitutive equations for granular geophysical flows [75].

\section{Theoretical concepts}

\subsection{Continuum description}

Plasticity and visco-plasticity are closely related to the pioneering work done by Coulomb [63] and Bingham [43], respectively. In the earliest descriptions of simple-shear flow experiments involving bulk materials, several concepts were drawn, which are summarized here.

- Shear-rate dependence: in the Coulomb description, the shear stress $\tau$ is independent of the shear rate $\dot{\gamma}$, whereas a Bingham fluid exhibits a linear dependence on the shear rate $\dot{\gamma}$ (see Fig. $5)$.

- Normal-stress dependence: the shear stress $\tau$ is a linear function of the normal stress $\sigma$ for a Coulomb material, whereas the shear stress $\tau$ is independent of the normal stress for a Bingham fluid.

- Two-phase flow effects: a Bingham fluid typically behaves like a one-phase homogeneous material, i.e., a single constitutive equation is sufficient to describe the bulk properties. In contrast, a water-saturated Coulomb material is a two-phase

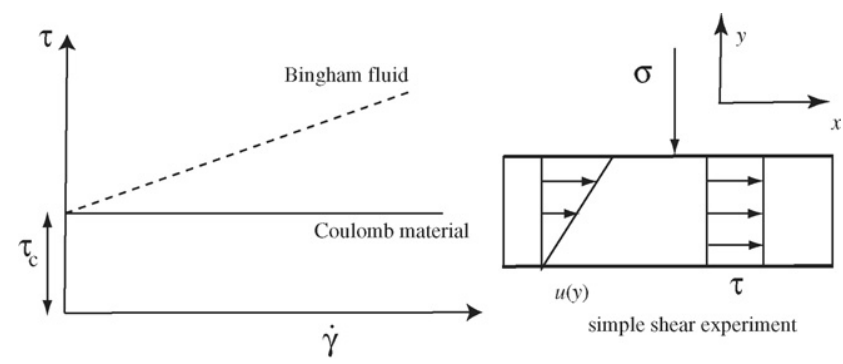

Fig. 5. Flow curve: material response for a Coulomb material (solid line) and Bingham fluid (dashed line) when the material undergoes a simple shear flow. 
material, with a separate response from the interstitial fluid and the solid phase. This observation leads to splitting the normal stress as an effective stress $\sigma^{\prime}$ and pore pressure $p$, a decomposition known as Terzaghi's decomposition principle [182,234]:

$\sigma=p+\sigma^{\prime}$.

- Yielding: when the shear stress is below a threshold $\tau_{\mathrm{c}}$, Coulomb and Bingham materials behave like rigid or elastic bodies. For shear stresses in excess of $\tau_{\mathrm{c}}$, the material yields and starts flowing

$$
\dot{\gamma}>0 \Rightarrow \tau=\tau_{\mathrm{c}}+\mu \dot{\gamma}^{n},
$$$$
\dot{\gamma}=0 \Rightarrow \tau \leq \tau_{\mathrm{c}} \text {, }
$$

with $\mu=0$ and $\tau_{\mathrm{c}}=\sigma^{\prime} \tan \varphi$ for a Coulomb material with $\tan \varphi$ the bulk friction angle, whereas $n=1$ and $\tau_{\mathrm{c}}=$ constant for a Bingham fluid. For viscoplastic materials, there are alternative phenomenological expressions such as the Casson model or the Herschel-Bulkley model [44].

Bingham and Coulomb materials are idealized representations of true materials. They have little in common except for the existence of a yield stress that separates a rigid/elastic domain and a fluid domain. These one-dimensional models are quite easy to understand and require little mathematics to be properly formulated. In contrast, their three-dimensional representation in the form of a tensorial expression needs much more work. Indeed, there are a number of rules that must be checked for a tensorial constitutive equation to be considered as admissible from the continuum-mechanics point of view $[123,184,230]$. The most important principle is material indifference: a physical law does not depend on a particular frame of reference. This leads to using quantities that remain invariant under any frame change. For instance, when referring to a particular stress state at a given point $M$ within the bulk, we can use the principal stresses (i.e., the eigenvalues $\sigma_{\mathrm{i}}$ of the stress tensor at $\mathrm{M}$ ) or the stress-tensor invariants. Principal stresses and stress-tensor invariants are both objective quantities, but stress-tensor invariants are more appropriate to interpreting the stress state. There are three stress-tensor invariants that can be defined in various ways since any combination of invariants is in turn an invariant quantity. To interpret them physically, we define them as follows [78,234]:

- The first invariant $I_{1}=\operatorname{tr} \sigma=\sigma_{1}+\sigma_{2}+\sigma_{3}$ represents the mean stress multiplied by 3 ( $|\mathbf{O P}|=I_{1} / \sqrt{3}$ in Fig. 6$)$. Point $\mathrm{P}$ is the orthogonal projection of the stress-state point M onto the trisectrix. For a simple fluid, the first invariant coincides with the fluid pressure (this statement does not hold for Coulomb materials).

- The second invariant $I_{2}=(1 / 2)\left(\operatorname{tr}\left(\sigma^{2}\right)-(\operatorname{tr} \sigma)^{2} / 3\right)=(1 / 2)$ $\operatorname{tr}\left(\mathbf{s}^{2}\right)$ can be interpreted as the deviation of a stress state from the mean stress state $\left(|\mathbf{P M}|^{2}=2 I_{2}\right.$ in Fig. 6) and is accordingly referred to as the deviator. We have introduced $\mathrm{s}=\sigma-I_{1} \mathbf{1} / 3$, which is called the extra-stress tensor or stress deviator.

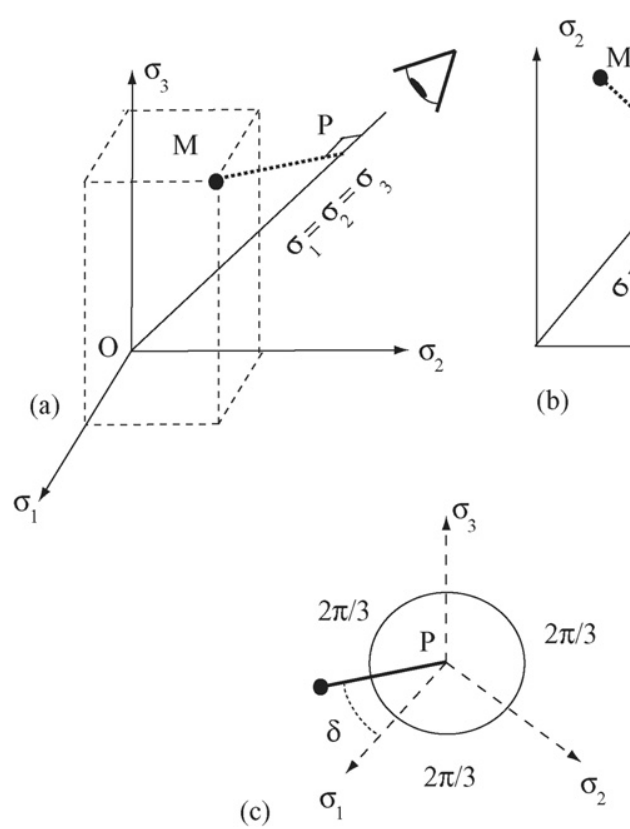

Fig. 6. Principal-stress space and interpretation of the representation in terms of invariants. (a) Representation in a three-dimensional space. The trisectrix represents equilibrium states. It has $\mathbf{n}=(1,1,1) / \sqrt{3}$ as the unit vector in the stress space. The distance OP is then given by computing $\mathbf{O M} \cdot \mathbf{n}=I_{1} / \sqrt{3}$. The deviatoric stress is the departure from equilibrium state. It is represented by the vector $\mathbf{P M}=\mathbf{O M}-\mathbf{O P}=\mathbf{O M}-I_{1} \mathbf{n} / \sqrt{3}$, giving $|P M|=\sqrt{2 I_{2}}$. (b) Representation in a two-dimensional space. (c) Deviatoric plane. This plane is orthogonal to the trisectrix and crosses it at $\mathrm{P}$. The dashed lines represent the orthogonal projection of the principal axes onto the deviatoric plane. The phase angle $\delta$ is reported.

- The third invariant $I_{3}=(1 / 3) \operatorname{tr} \mathbf{s}^{3}$ represents the angle in the deviatoric plane (i.e., the plane orthogonal to the trisectrix at M) of the vector PM with respect to the projection of a fixed direction onto the deviatoric plane. This invariant is sometimes called the phase or Lode's angle $\cos ^{2} 3 \delta=27 I_{3}^{2} /\left(4 I_{2}^{3}\right)$.

Let us assume that we apply an isotropic stress state to the material. In the stress space, the stress point is a point $\mathrm{M}$ along the trisectrix $\sigma_{1}=\sigma_{2}=\sigma_{3}$. In this case, the material never fails. If we now carry out simple shear experiments (see Fig. 5), the stress tensor has the following components and invariants

$\boldsymbol{\sigma}=\left[\begin{array}{ccc}\sigma & \tau & 0 \\ \tau & \sigma & 0 \\ 0 & 0 & \sigma\end{array}\right], \quad I_{1}=3 \sigma, I_{2}=\tau, \quad$ and $\quad I_{3}=0$,

in a Cartesian frame $(x, y)$. This means that we force the material to depart orthogonally from the line $\sigma_{1}=\sigma_{2}=\sigma_{3}$, when applying a deviatoric stress $\tau$ to the material. According to the experimental observations, if the shear stress exceeds a critical value, the material yields. The yielding condition must then be expressed, at least, as a function of the second stress-tensor invariant $I_{2}$. In the stress space $\left(\sigma_{1}, \sigma_{2}, \sigma_{3}\right)$, there is a surface delimiting two possible mechanical states of a material element, as depicted in Fig. 7. The surface is referred to as the yield surface and is usually represented by an equation in the form $f\left(I_{1}, I_{2}, I_{3}\right)=$ 


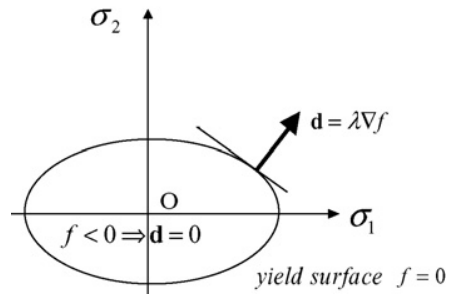

Fig. 7. Yield surface delimiting two domains: $f<0$ solid (rigid or elastic) domain, $f>0$ fluid domain. The equation $f=0$ represents the yield surface.

0 , where $f$ is called the plastic rule. When $f<0$, behavior is generally assumed to be elastic or rigid. When $f=0$, the material yields. When $f>0$, the material behaves like a fluid.

In plasticity, the simplest yield criterion is the von Mises criterion, stating that yield occurs whenever the deviator exceeds a critical value (whose root gives the yield stress):

$f\left(I_{2}\right)=\sqrt{I_{2}}-\tau_{\mathrm{c}}$.

As depicted in Fig. 8, the yield surface is a cylinder of radius $\tau_{\mathrm{c}}$ centered around the axis $\sigma_{1}=\sigma_{2}=\sigma_{3}$. The simplest plastic rule consistent with the one-dimensional Coulomb law is the Drucker-Prager rule, for which the yield surface is a cone around the trisectrix $\sigma_{1}=\sigma_{2}=\sigma_{3}$, with its apex at the origin $\mathrm{O}$

$f\left(I_{1}, I_{2}\right)=\sqrt{I_{2}}-k I_{1}$,

with $k=(1 / 3) \sin \varphi>0$ a constant. In the Drucker-Prager rule, the yield surface is axisymmetric. There are more complicated rules, in which the third invariant plays a role, such as the MohrCoulomb plastic rule, the representation of which in the stress space is an irregular hexagonal pyramid [234]:

$f\left(I_{1}, I_{2}, I_{3}\right)=\sqrt{I_{2}}\left[\sin \left(\delta+\frac{\pi}{3}\right) k \sqrt{3} \cos \left(\delta+\frac{\pi}{3}\right)\right]-k I_{1}$,

where we have expressed the third invariant in terms of Lode's angle $\delta$.

We have so far answered to the question: for which stress conditions does the material yield? We now have to respond to the question: what does it happen after yielding? We assume that, after yielding, the following principles hold.

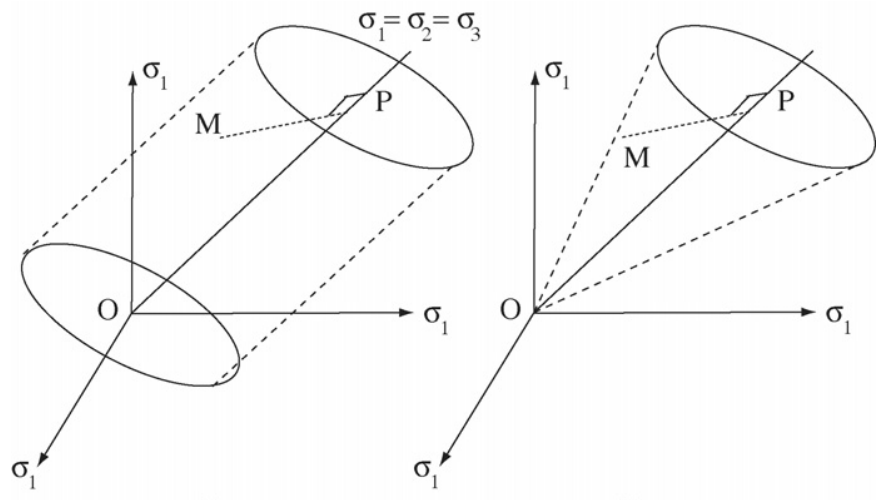

(a)

(b)

Fig. 8. Representation of the yield surface in the principal stress space. (a) Von Mises yield surface and (b) Drucker-Prager yield surface. (i) Coaxiality principle: the principal directions of the extrastress and strain-rate tensors coincide since the material moves in reaction to the solicitation.

(ii) Associate normal flow rule: the strain-rate tensor is directly proportional to the surplus of stress, that is, the distance between the point representing the stress state and the yield surface, i.e. $\sqrt{I_{2}}-\tau_{\mathrm{c}}$.

Translated into mathematical terms, principles (i) and (ii) lead to: $\mathbf{d}=\lambda\left(\sqrt{I_{2}}-\tau_{\mathrm{c}}\right) \nabla f$, with $\lambda$ a proportionality coefficient (Lagrangian multiplier), when $f>0$. We obtain

$$
\begin{aligned}
\mathbf{d} & =\frac{\lambda}{2}\left(\sqrt{I_{2}}-\tau_{\mathrm{c}}\right) \frac{\mathbf{s}}{\sqrt{I_{2}}} \text { when } f>0, \\
& =0 \text { when } f \leq 0 .
\end{aligned}
$$

Note that in plastic potential theory, we could also imagine other flow rules, e.g., once the material has yielded $(f=0)$, the deformation derives from a potential $F$ that differs from $f$; in that case, the flow rule is said to be non-associate and $\mathbf{d}=$ $\lambda \nabla F$. Here, we have used the simplest arguments to reply to the question above, as Prager [115,196] and Oldroyd [183] did for Bingham fluids. It should, however, be remembered that the behavior of true materials is usually much more complicated [153].

We can invert Eq. (3) by computing $\mathbf{d} \cdot \mathbf{d}$ and then taking the trace. We obtain $\operatorname{tr}\left(\mathbf{d}^{2}\right)=\lambda^{2}\left(\sqrt{I_{2}}-\tau_{\mathrm{c}}\right)^{2} / 2$. Defining the second invariant of the strain-rate tensor as $J_{2}=(1 / 2) \operatorname{tr}\left(\mathbf{d}^{2}\right)$, we then derive

$\mathbf{s}=\frac{1}{\lambda} \frac{\sqrt{J_{2}}+\tau_{\mathrm{c}}}{\sqrt{J_{2}}} \mathbf{d}$ when $f>0$.

For this equation to be consistent with the phenomenological relation (2), we must set $\lambda^{-1}=\mu$. We finally obtain the constitutive equation in a tensorial form for a Bingham fluid

$\mathbf{s}=\left(2 \mu+\frac{\tau_{\mathrm{c}}}{\sqrt{J_{2}}}\right) \mathbf{d}$ when $f>0$,

$\mathbf{s}=0$ when $f \leq 0$.

The same exercise can be repeated for the Drucker-Prager yield surface. We obtain

$\mathbf{d}=\frac{\lambda}{2}\left(\frac{\mathbf{s}}{\sqrt{I_{2}}} f-2 f k \mathbf{1}\right)$ when $f>0$,

with $f=\sqrt{I_{2}}-k I_{1}$. We can notice that the first invariant of the strain-rate tensor is nonzero since $J_{1}=-\operatorname{trd}=3 f \lambda k>0$, which implies that the bulk volume increases indefinitely with time (dilatancy of the material), which is not realistic. The second invariant of the strain-rate tensor is $J_{2}=(1 / 2) \operatorname{tr}\left(\mathbf{d}^{\prime 2}\right)=$ $(\lambda / 2)^{2} f^{2}$, where we split the strain-rate tensor into a deviatoric (traceless) contribution $\mathbf{d}^{\prime}$ and an isotropic term $\mathbf{d}^{\prime \prime}$. We can then relate the deviatoric contributions of the stress tensor and the deviatoric part of the strain-rate tensor

$\mathbf{s}=\left(k I_{1}+\frac{J_{1}}{3 \lambda k}\right) \frac{\mathbf{d}^{\prime}}{\sqrt{J_{2}}} \quad$ when $f>0$.

We verify that, when an isochoric simple shear is applied to the material (i.e., $J_{2}=\dot{\gamma}$ and $J_{1}=0$, see Fig. 5), the shear stress 
is linearly dependent on the normal stress and independent of the shear rate: $\tau=3 k \sigma$, consistently with the phenomenological Coulomb law. It is worth noting that this derivation of the bulk stress tensor in Eq. (5) is purely formal since the bulk stress tensor conflicts with experimental observations, except for the prediction of a Coulomb behavior for simple-shear flow conditions. Indeed, an obvious shortcoming has been seen just above with dilatancy, which imposes modifying the yield function $f$ to take material compressibility into account $[75,89,160,234]$.

The last point in this presentation of continuum-mechanics tools concerns fluid-solid coupling. For viscoplastic materials, the coupling is complete since the suspension behaves as a whole on the bulk scale. On the contrary, fluid-saturated coarse-grained Coulomb materials behave as two-phase materials on the bulk scale, i.e., the solid and interstitial-fluid phases may move separately at different velocities. The Terzaghi principle states that the bulk stress tensor can be divided into a fluid contribution (pore pressure) and a solid contribution (reflecting the force distribution within the granular skeleton) (see Eq. (1)). Most often, the viscous effects of the interstitial fluid can be neglected so that the fluid action reduces to a pressure term. This pressure can be hydrostratic if the relative velocity $\mathbf{v}$ between the two phases is zero or nearly zero. When $\mathbf{v}$ is slightly nonzero, there is a pressure gradient within the bulk due to the fluid sewage, which can be described using the linear Darcy law

$\nabla p=-\frac{\mu}{\kappa} \mathbf{v}$

where $\kappa$ is the permeability coefficient, which is a function of both the particle radius $a$ and the solid concentration $\phi$. The Kozeny-Carman relation can be used to evaluate this coefficient: $\kappa=a^{2}(1-\phi) /\left(45 \phi^{2}\right)$, with $\phi$ the solid concentration $(\phi$ is the volume occupied by particles to total volume). This relation shows that the pore pressure is sensitive to the particle size and changes in the solid concentration. Note also that shearing a coarse-grained material usually leads to a bulk volume increase (dilatancy), which induces a decrease in the solid concentration, thus an increase in the permeability coefficient $\kappa$. For materials vigorously sheared, a more complex diffusion equation must be used $[128,129,213]$. This equation relates the pressure gradient and the total derivative of the solid concentration

$\frac{\mu}{\kappa \phi} \frac{\mathrm{d} \phi}{\mathrm{d} t}=-\left(\phi \nabla-\frac{\rho_{\mathrm{f}}}{\bar{\rho}} \nabla \phi\right) \cdot \nabla p$,

with $\bar{\rho}=\phi \rho_{\mathrm{p}}+(1-\phi) \rho_{\mathrm{f}}$ the bulk density. As pointed out by Iverson $[128,129]$, this equation is crucial since it shows how high fluid pressure can be generated in a dilating/contracting granular material and how this alteration in the pore pressure influences the frictional behavior (see Eq. (1)).

\subsection{Averaged balance equations}

We are now seeking why some granular materials behave like a one-phase material on the bulk scale and why there are different types of yield surface. For this purpose, we consider suspensions of equal-size, spherical particles and outline the basic elements in microstructural theories of particle suspensions needed for deriving the governing equations and the bulk stress tensor.

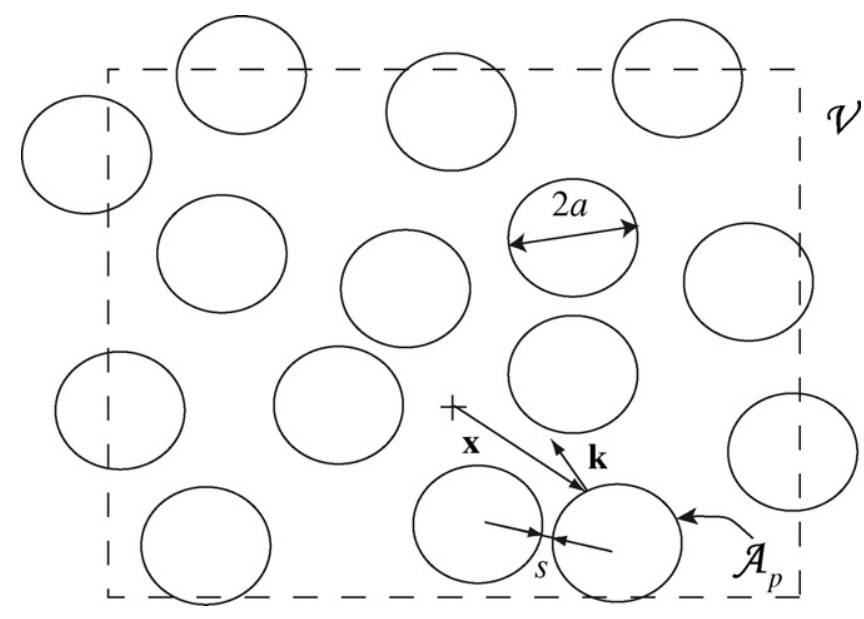

Fig. 9. Idealized suspension of spherical particles in a Newtonian fluid.

In any microstructural approach to particle suspensions, the starting point is to examine the behavior of individual components on a particle scale, then to infer the bulk rheological behavior by using an appropriate average process. In order to avoid overly general explanations, we assume that (see Fig. 9)

(1) the interstitial fluid is Newtonian, with viscosity $\mu$ and density $\rho_{\mathrm{f}}$;

(2) the particles are rigid, spherical, and of equal size (radius $a$, density $\rho_{\mathrm{p}}$ ).

The suspension is assumed to be statistically homogeneous. The number of particles per unit volume (density number) is $n$ and is related to the solid concentration $\phi$ since we have $n=\phi /\left(4 \pi a^{3} / 3\right)$.

Interstitial fluid motion is described by the Navier-Stokes equations

$\frac{\partial \mathbf{u}_{\mathrm{f}}}{\partial t}+\left(\mathbf{u}_{\mathrm{f}} \cdot \nabla\right) \mathbf{u}_{\mathrm{f}}=-\frac{1}{\rho_{\mathrm{f}}} \nabla p+\frac{1}{\rho_{\mathrm{f}}} \nabla \cdot \sigma_{\mathrm{f}}$,

$\nabla \cdot \mathbf{u}_{\mathrm{f}}=0$

where $\mathbf{u}_{\mathrm{f}}$ is the fluid velocity, $p$ the generalized pressure (including the fluid pressure and gravity potential), and $\sigma_{\mathrm{f}}$ is the stress tensor (here $\sigma_{\mathrm{f}}=2 \mu \mathbf{d}$ where $\mathbf{d}$ denotes the strain-rate tensor). The equation of motion for the particle can be written in the following Lagrangian form

$\frac{\mathrm{d} \mathbf{u}_{\mathrm{p}}}{\mathrm{d} t}=\mathbf{g}+\frac{1}{m_{\mathrm{p}}} \mathbf{F}\left(\mathbf{u}_{\mathrm{p}}, \mathbf{u}_{\mathrm{f}}\right)$,

where $\mathbf{F}\left(\mathbf{u}_{\mathrm{p}}, \mathbf{u}_{\mathrm{f}}\right)$ is the force field resulting from the interaction between the fluid and the particle, $m_{\mathrm{p}}$ the particle mass, and $\mathbf{u}_{\mathrm{p}}$ the velocity of the mass center. The boundary conditions at the solid/fluid interface reflect non-penetration and fluid adherence: $\mathbf{u}_{\mathrm{f}} \cdot \mathbf{k}=0$, where $\mathbf{k}$ denotes the outwardly-oriented unit normal. Note that

- the force field $\mathbf{F}\left(\mathbf{u}_{\mathrm{p}}, \mathbf{u}_{\mathrm{f}}\right)$ is not yet defined;

- when expressing the dependence of $\mathbf{F}$ on the flow variables, we assume that it depends on the instantaneous particle ve- 
locity and the (Eulerian) velocity field (given by the NavierStokes equations).

To obtain a more physical picture of the fluid/particle interplay, we introduce dimensionless numbers and transform the equations above into dimensionless expressions. Let us introduce a velocity scale $U_{*}$ for the fluid. The timescale for the fluid motion near the particle is then: $t_{\mathrm{f}}=a / U_{*}$. The characteristic time for the particle is defined as a relaxation time, that is, the time needed for its velocity to vary substantially as a result of the fluid action. If $F$ is the order of magnitude of the fluid-particle interaction, examining Eq. (9) leads to selecting: $t_{\mathrm{p}}=m_{\mathrm{p}} U_{*} / F$. The equations of motion can now be written in dimensionless form as follows (dimensionless variables have a tilde)

$R e_{\mathrm{p}}\left(\frac{\partial \tilde{\mathbf{u}}_{\mathrm{f}}}{\partial \tilde{t}}+\left(\tilde{\mathbf{u}}_{\mathrm{f}} \cdot \nabla\right) \tilde{\mathbf{u}}_{\mathrm{f}}\right)=-\frac{P_{*} \rho_{\mathrm{f}} a}{\mu U} \nabla \tilde{p}+\Delta \tilde{\mathbf{u}}_{\mathrm{f}}$,

where $P_{*}$ is the pressure scale [here $\left.P_{*}=\mu U_{*} /\left(\rho_{\mathrm{f}} a\right)\right]$ and

$R e_{\mathrm{p}}=\rho_{\mathrm{f}} U_{*} a / \mu$,

is the particle Reynolds number. For the particle, one obtains

$S t \frac{\mathrm{d} \tilde{\mathbf{u}}_{\mathrm{p}}}{\mathrm{d} \tilde{t}}=\frac{m_{\mathrm{p}}}{F} \mathbf{g}+\tilde{\mathbf{F}}\left(\tilde{\mathbf{u}}_{\mathrm{p}}, \tilde{\mathbf{u}}_{\mathrm{f}}\right)$,

where

$S t=\frac{t_{\mathrm{p}}}{t_{\mathrm{f}}}=\frac{m_{\mathrm{p}} U_{*}^{2}}{F a}$,

is called the Stokes number. Two asymptotic regimes can be achieved depending on the value of the Stokes number:

- $S t \gg 1$. The fluid has no time to adjust its velocity to the variations in the particle velocity and, conversely, the particle is not affected by the rapid variations in the fluid velocity (but naturally it continues to be affected by the slow variations). In practice, this means that the fluid and the particle evolve in a quasi-autonomous way and, therefore, their motion can be considered separately. On a macroscopic scale, such suspensions retain a genuinely two-phase character and the equations of motion take the form of two interrelated equations (one for each phase).

- $S t \rightarrow 0$. The particle has time to adjust its velocity to any change in the fluid velocity field. One sometimes says that the particle is the slave of the fluid phase. On a macroscopic scale, this means that the suspension behaves as a one-phase medium.

From this discussion, one must keep in mind that, if any particle suspension is a two-phase material on a particle scale, the suspension can behave as a one-phase fluid on a macroscopic scale. In addition, the only asymptotic regimes for which it is possible to deduce the fluid-particle interaction in a completely theoretical way are the regimes $S t \rightarrow 0$ and $R e_{\mathrm{p}} \rightarrow 0$ and $S t \rightarrow \infty$ and $R e_{\mathrm{p}} \rightarrow \infty[35,143,226]$.

After outlining the coupling between the solid and fluid phases, we derive the governing equations (mass and momentum balance equation) by averaging the local equations of motion.
We will emphasize the flow conditions for which it is possible to provide a rheological description within the framework of one-phase fluids. Another important point is the derivation of the bulk stress tensor.

\subsubsection{Bulk mass balance equation}

The approach involves taking the volume average of the local equations of motion (volume averaging). The operator "volume average" is constructed by taking a control volume $\mathcal{V}$ assumed to be sufficiently wide to contain a large number of articles, but in the meantime sufficiently small with the respect to a typical lengthscale of the bulk for it to be considered a continuum.

For the solid and fluid phases, the local mass balance equation is in the form

$\frac{\partial \rho_{\mathrm{i}}}{\partial t}+\nabla \cdot\left(\rho_{\mathrm{i}} \mathbf{u}\right)=0$

with $\mathrm{i}=\mathrm{p}$ (particle) or $\mathrm{i}=\mathrm{f}$ (fluid) and where $\mathbf{u}$ denotes the local velocity coinciding with fluid velocity within the continuous phase and the solid velocity within a particle. We define the bulk (volume-averaged) density as $\bar{\rho}=\phi \rho_{\mathrm{p}}+(1-\phi) \rho_{\mathrm{f}}$ with $\phi$ the solid concentration. We also define a characteristic function [154]

- $H(\mathbf{x})=1$ if $\mathbf{x}$ is inside a particle,

- $H(\mathbf{x})=0$ if $\mathbf{x}$ lies within the fluid.

The characteristic function is locally discontinuous (at the fluid/solid interface) and must be considered as a generalized function. Using distribution theory, we can show that

$$
\frac{\partial H_{\mathrm{i}}}{\partial t}+\mathbf{u} \cdot \nabla\left(H_{\mathrm{i}}\right)=0
$$

with $\mathrm{i}=\mathrm{f}$ or $\mathrm{p}$ and where the following short-hand notations $H_{\mathrm{p}}=H$ and $H_{\mathrm{f}}=1-H$ have been used. This equation is sometimes referred to as the topological equation [85]. Note that we have $\int_{\mathcal{V}} H \mathrm{~d} \mathcal{V}=\phi \mathcal{V}$.

We call $\mathcal{V}_{\mathrm{p}}$ the sub-volume of $\mathcal{V}$ containing the particles and $\mathcal{A}_{\mathrm{p}}$ the surface bounding $\mathcal{V}_{\mathrm{p}}$. Multiplying the mass equation for solid particles by the characteristic function $H$ and the mass equation for the continuous phase by $1-H$ and integrating over the control volume $\mathcal{V}$, we obtain

$$
\begin{aligned}
& \int_{\mathcal{V}}\left(H \frac{\partial \rho_{\mathrm{p}}}{\partial t}+(1-H) \frac{\partial \rho_{\mathrm{f}}}{\partial t}+H \nabla \cdot\left(\rho_{\mathrm{p}} \mathbf{u}\right)+(1-H) \nabla \cdot\left(\rho_{\mathrm{f}} \mathbf{u}\right)\right) \\
& \mathrm{d} \mathcal{V}=0,
\end{aligned}
$$

which can be transformed into

$$
\begin{aligned}
& \int_{\mathcal{V}}\left(\frac{\partial \bar{\rho}}{\partial t}+\nabla \cdot(\bar{\rho} \mathbf{u})-\rho_{\mathrm{p}} \frac{\partial H}{\partial t}-\rho_{\mathrm{f}} \frac{\partial(1-H)}{\partial t}-\rho_{\mathrm{p}} \nabla \cdot(H \mathbf{u})\right. \\
& \left.\quad-\rho_{\mathrm{f}} \nabla \cdot((1-H) \mathbf{u})\right) \mathrm{d} \mathcal{V}=0 .
\end{aligned}
$$

Then, applying the Gauss and Leibnitz rules to interchange the time/space derivatives with the volume averaging operator, we deduce the bulk mass equation

$$
\frac{\partial \bar{\rho}}{\partial t}+\nabla \cdot(\bar{\rho} \mathbf{u})=0 .
$$


As expected, this result shows that the bulk mass balance equation satisfies the same classic law as the solid and fluid phases do.

\subsubsection{Bulk momentum balance equation}

Multiplying Eq. (7) with $1-H$, then integrating it over the control volume $\mathcal{V}$, making use of the topological Eq. (12), the relation $\nabla H=\mathbf{k}$ over $\mathcal{A}_{\mathrm{p}}$ with $\mathbf{k}$ the unit outward normal to $\mathcal{A}_{\mathrm{p}}$ (and $\nabla H=0$ otherwise), and the Reynolds decomposition $\mathbf{u}_{\mathrm{f}}=\overline{\mathbf{u}}_{\mathrm{f}}+\mathbf{u}_{\mathrm{f}}^{\prime}$, we eventually obtain

$$
\begin{aligned}
\rho_{\mathrm{f}}\left(\frac{\partial \overline{\mathbf{u}}_{\mathrm{f}}}{\partial t}+\nabla \cdot \overline{\mathbf{u}}_{\mathrm{f}} \overline{\mathbf{u}}_{\mathrm{f}}\right)= & -\nabla \bar{p}+\frac{1}{\mathcal{V}} \int_{\mathcal{A}_{\mathrm{p}}}\left(\sigma_{\mathrm{f}}-p \mathbf{1}\right) \cdot \mathbf{k} \mathrm{d} \mathcal{A} \\
& +\nabla \cdot \frac{1}{\mathcal{V}} \int_{\mathcal{V}_{\mathrm{f}}}\left(\sigma_{\mathrm{f}}-\rho_{\mathrm{f}} \mathbf{u}_{\mathrm{f}}^{\prime} \mathbf{u}_{\mathrm{f}}^{\prime}\right) \mathrm{d} \mathcal{V} .
\end{aligned}
$$

In this equation, the mean fluid velocity is

$\overline{\mathbf{u}}_{\mathrm{f}}(\mathbf{x}, t)=\frac{1}{\mathcal{V}} \int_{\mathcal{V}}(1-H(\mathbf{x}, t)) \mathbf{u}_{\mathrm{f}}(\mathbf{x}, t) \mathrm{d} \mathcal{V}=\frac{1-\phi}{\mathcal{V}_{\mathrm{f}}} \int_{\mathcal{V}} \mathbf{u}_{\mathrm{f}}(\mathbf{x}, t) \mathrm{d} \mathcal{V}$, where we used $\int_{\mathcal{V}}(1-H) \mathrm{d} \mathcal{V}=(1-\phi) \mathcal{V}$. Here, the mean fluid velocity is $1-\phi$ the mean bulk velocity.

For the solid phase, we have to transform the Lagrangian equation of motion (9) into an Eulerian equivalent. The rigidsphere assumption implies that the solid kinematic field is: $\mathbf{u}_{\mathrm{p}}(\mathbf{x}, t)=\mathbf{u}_{\mathrm{p}}(\mathbf{y}, t)+\omega_{\mathrm{p}} \times(\mathbf{x}-\mathbf{y})$, with $\mathbf{y}$ the position of the center of mass and $\omega_{\mathrm{p}}$ the rotation velocity. Since the gradient of a rotational field is zero, the local equation is for a solid particle: $\rho_{\mathrm{p}} \partial \mathbf{u}_{\mathrm{p}} / \partial t=\rho_{\mathrm{p}} \mathbf{g}+\nabla \cdot \boldsymbol{\sigma}_{\mathrm{p}}$, with the following difficulty: the particle being rigid, the stress field is undetermined. This is in fact a minor issue since we will integrate the stress field over the particle volume and the Green-Ostrogradski allows us to connect this field to the forces acting on the particle surface. Proceeding as earlier by multiplying the equation above by $H$, then integrating it on $\mathcal{V}$, we find

$$
\begin{aligned}
\rho_{\mathrm{p}}\left(\frac{\partial \overline{\mathbf{u}}_{\mathrm{p}}}{\partial t}+\nabla \cdot \overline{\mathbf{u}}_{\mathrm{p}} \overline{\mathbf{u}}_{\mathrm{p}}\right)= & \phi \rho_{\mathrm{p}} \mathbf{g}+\nabla \cdot\left(\overline{\boldsymbol{\sigma}}_{\mathrm{p}}-\rho_{\mathrm{p}} \overline{\mathbf{u}_{\mathrm{p}}^{\prime} \mathbf{u}_{\mathrm{p}}^{\prime}}\right) \\
& -\nabla \cdot \frac{1}{\mathcal{V}} \int_{\mathcal{A}_{\mathrm{p}}} \boldsymbol{\sigma}_{\mathrm{p}} \cdot \mathbf{k} \mathrm{d} \mathcal{A},
\end{aligned}
$$

where again we used the Reynolds decomposition $\mathbf{u}_{\mathrm{p}}=\overline{\mathbf{u}}_{\mathrm{p}}+$ $\mathbf{u}_{\mathrm{p}}^{\prime}$. Since at the particle surface we have $\sigma_{\mathrm{p}} \cdot \mathbf{k}=\left(\sigma_{\mathrm{f}}-p \mathbf{1}\right) \cdot \mathbf{k}$, the last term on the right-hand side in Eq. (14), representing the stresses exerted on the particle surface, is equivalent to the term in Eq. (13). These terms reflect momentum transfer between the two phases through their interface.

The local bulk velocity is defined as follows: $\mathbf{u}(\mathbf{x}, t)=$ $H \mathbf{u}_{\mathrm{p}}(\mathbf{x}, t)+(1-H) \mathbf{u}_{\mathrm{f}}(\mathbf{x}, t)$. The bulk volume-averaged velocity is then: $\overline{\mathbf{u}}(\mathbf{x}, t)=\overline{\mathbf{u}}_{\mathrm{p}}(\mathbf{x}, t)+\overline{\mathbf{u}}_{\mathrm{f}}(\mathbf{x}, t)$. We can also define a bulk velocity based on mass averaging (rather than volume): $\bar{\rho} \overline{\mathbf{u}}_{\mathrm{m}}=\rho_{\mathrm{p}} \overline{\mathbf{u}}_{\mathrm{p}}+\rho_{\mathrm{f}} \overline{\mathbf{u}}_{\mathrm{f}}$, with $\bar{\rho}=\phi \rho_{\mathrm{p}}+(1-\phi) \rho_{\mathrm{f}}$. The two velocities coincide when the solid and fluid densities are equal. A helpful approximation can be used when one of the densities is very low compared to the other and the velocities of each phase are of the same magnitude. Using the same dimensional argument as earlier, we can show that we meet this case for a suspension of particles within a gas with $S t \gg 1$ and $\rho_{\mathrm{p}} \gg \rho_{\mathrm{f}}$; the same situation is met with emulsions $\left(\rho_{\mathrm{p}} \ll \rho_{\mathrm{f}}\right.$ and $\left.S t \rightarrow 0\right)$. Note that the mass conservation is satisfied $\nabla \cdot \overline{\mathbf{u}}=\nabla \cdot \overline{\mathbf{u}}_{\mathrm{m}}=0$ ( $\phi$ is assumed to be constant).

Summing (13) and (14) leads to the bulk momentum equation $\bar{\rho}\left(\frac{\partial \overline{\mathbf{u}}_{\mathrm{m}}}{\partial t}+\nabla \cdot \overline{\mathbf{u}}_{\mathrm{m}} \overline{\mathbf{u}}\right)=-\nabla \bar{p}^{\star}+\nabla \cdot \frac{1}{\mathcal{V}} \int_{\mathcal{V}}\left(\sigma-\rho \mathbf{u}^{\prime} \mathbf{u}^{\prime}\right) \mathrm{d} \mathcal{V}$,

with $\bar{p}^{\star}=\bar{\Phi}+\bar{p}_{\mathrm{f}}$ (where $\nabla \bar{\Phi}=-\bar{\rho} \mathbf{g}$ ). This equation is not very helpful as long as we are not able to transform it into the classic form of a momentum balance equation for a continuum; here this means that we must have $\overline{\mathbf{u}}_{\mathrm{m}} \approx \overline{\mathbf{u}}$ so that the terms on the left-hand side of Eq. (15) can be identified as a material derivative. If this condition is satisfied, then we can identify the term on the right-hand side under the divergence operator as a stress tensor. We refer to it as the bulk extra-stress tensor

$\overline{\boldsymbol{\sigma}}=\frac{1}{\mathcal{V}} \int_{\mathcal{V}}\left(\sigma-\rho \mathbf{u}^{\prime} \mathbf{u}^{\prime}\right) \mathrm{d} \mathcal{V}$,

which is the definition used by [34] for the bulk stress tensor. Further computation reveals that this stress tensor can be divided into a fluid contribution [14,34]

$\overline{\boldsymbol{\sigma}}^{(\mathrm{f})}=2 \mu \overline{\mathbf{d}}-\frac{1}{\mathcal{V}} \int_{\mathcal{V}_{\mathrm{f}}} \rho_{\mathrm{f}} \mathbf{u}^{\prime} \mathbf{u}^{\prime} \mathrm{d} \mathcal{V}$,

and a solid contribution [14]

$\overline{\boldsymbol{\sigma}}^{(\mathrm{p})}=\frac{1}{\mathcal{V}} \int_{\mathcal{A}_{\mathrm{p}}} \boldsymbol{\sigma} \cdot \mathbf{x k} \mathrm{d} \mathcal{A}-\frac{1}{\mathcal{V}} \int_{\mathcal{V}_{\mathrm{p}}} \rho_{\mathrm{p}} \mathbf{u}^{\prime} \mathbf{u}^{\prime} \mathrm{d} \mathcal{V}+\mathbf{G}\left(\omega_{\mathrm{p}}\right)$,

where $\mathbf{G}\left(\omega_{\mathrm{p}}\right)$ represents an antisymmetric function $\omega_{\mathrm{p}}$, which is not detailed here because in most cases of practical interest, $\mathbf{G}$ vanishes $[14,34,198]$.

\subsection{Constitutive equations: physical origin of the yield stress}

In the derivation of the bulk momentum equation, we have found that the solid contribution in Eq. (18) can be defined as

$\overline{\boldsymbol{\sigma}}^{(\mathrm{p})} \approx \frac{1}{\mathcal{V}} \int_{\mathcal{A}_{\mathrm{p}}} \boldsymbol{\sigma} \cdot \mathbf{x k} \mathrm{d} \mathcal{A}=\frac{a}{\mathcal{V}} \int_{\mathcal{A}_{\mathrm{p}}} \mathbf{f k} \mathrm{d} \mathcal{A}$,

where $\mathbf{f}=\sigma \cdot \mathbf{k}$ is the stress at the particle surface, when the influence of particle velocity fluctuations can be neglected. This definition is quite general and can be found in soil mechanics $[60,109,176]$, homogeneization theory [51,52], and rheology of particle suspensions [14,34]. For concentrated particle suspensions, the stress state at the particle surface is directly related to interparticle interactions. Flow initiation or yielding is then directly a consequence of changes in these interactions. In rheology, three classes of particle interaction are usually considered: colloidal interaction, lubricated contact, and direct (frictional and/or collisional) contact $[1,66]$. Here, we address the specific issue of the yield stress computation for suspensions made up of particles with nearly the same size.

For colloidal interactions, a vast literature has been published about the influence of colloidal interactions on the bulk constitutive equation $[205,206]$. For dilute and moderately concentrated 
suspensions and low Péclet numbers, $P e=\Phi / k T$, with $\Phi$ the particle energy potential (depending on electrostatic forces and function of arrangement, ionic strength, Debye length, solid fraction, etc.), $k$ Boltzmann's constant, and $T$ absolute temperature, solid particles are permanently fluctuating and never reach an equilibrium position because of Brownian effects. In this case, the bulk behavior is close to that of a dilute suspension of noncolloidal particles; there is no yield stress [37,46,233]. At high Péclet numbers, the particles find an equilibrium position (at least after a possibly long rest period) and cannot easily move away from each other. In that case, the suspension exhibits a yield stress since flow can be obtained only if a finite energy is provided to the system to extract each particle from its instantaneous local potential [135].

For a number of colloidal suspensions including natural materials, the situation is somewhat different because particles are not electrically stabilized and form aggregates (or flocs), which makes their rheological characterization more difficult [240]. Potanin et al. [193,194] developed a phenomenological fractal model to determine bulk behavior of weakly aggregated dispersions. They assumed that particles form aggregates which in turn are connected into a network. Thus they interpreted bulk yield stress as a consequence of chain breakup due to thermal fluctuations and rupture under compressive force. Another conceptual model inspired by glassy dynamics has been proposed by Sollich et al. [91,224,225]. They showed that the bulk mechanical properties can be related to the internal structure (described in terms of the particle energy distribution). To date such models are able to mimic bulk behavior over a wide range of flow conditions, but cannot specify the effects of particle size, size distribution, or solid concentration on the yield stress of a particulate fluid. Kapur et al. [138], and then Scales et al. [215] proposed a mean-field theory for particles governed by the van der Waals attractive forces. The input values of the model were the Hamaker constant $A$, the coordination number $C_{N}$, the mean particle diameter $2 a$, and an interparticle separation parameter $s_{0}$, which must be fitted from experimental data. The yield stress is computed as the summation of all pairwise interparticle forces (per unit area). More recently, on the same basis, Zhou et al. improved this model by taking into account a broader size distribution of particles, but limited their attention to systems at the isoelectric point [247]. They found that the maximum yield stress can be written as

$\tau_{k}(\phi)=K\left(\frac{\phi}{1-\phi}\right)^{c} \frac{1}{(2 a)^{2}}$

where $K=3.1 \mathrm{Ab} /\left(24 \pi s_{0}\right)$, and $b$ and $c$ are two parameters to be fitted from experimental data. They proposed the following explanation for the variation in yield stress with increasing solid concentration. A weakly flocculated dispersion may be seen as a series of weakly interconnected aggregates (flocs) made up of strongly interacting particles. At low solid concentrations, yielding results from the breakdown of the weak links between flocs. At high solid concentrations, yielding is a consequence of the rupture of interparticle bonds and resistance to the deformation of networks. This means that a critical solid concentration $\phi_{\mathrm{c}}$ separating the two domains should exist.
- When $\phi<\phi_{\mathrm{c}}$, structural effects due to weak links between flocs prevail over those due to geometric resistance and the yield stress varies with a solid concentration such as $\tau_{k} \approx$ $K \phi^{c} / d^{2}$. This effect is included in Eq. (20) since it can be derived from Eq. (20) by taking a series expansion to the chief order at $\phi=0$.

- When $\phi>\phi_{\mathrm{c}}$, the geometric resistance becomes more pronounced, resulting in a much higher dependence on the solid concentration $\tau_{k} \approx K \phi^{c^{\prime}} / d^{2}$, with $c^{\prime}>c$. Zhou et al. considered that from a microstructural point of view, the geometric resistance enhancement is reflected by the increase in particle contacts. Assuming that the coordination number is given by Rumpf's expression $\left(C_{N}=3.1 /(1-\phi)\right)$, they arrived at the conclusion that the yield stress may be scaled as a power function of $\phi /(1-\phi)$. The series expansion at $\phi=0$ implies that the exponent must be $c$. Moreover, their experiments with alumina suspensions showed that the critical solid concentration ranged from 0.26 to 0.44 and depended on the particle diameter.

In noncolloidal systems, particles experience direct (i.e., sustained frictional) or lubricated contacts. When particles experience sustained frictional contacts, particle friction gives rise to the Coulomb yielding process on the bulk scale, characterized by a linear relationship between the normal and shear stresses: $\tau=\tan \varphi \sigma$. It has long been stated that the bulk Coulomb law on the bulk scale was a direct consequence of the Coulomb frictional behavior on the particle scale. In fact, the link between the two scales is not particularly direct. Using Eq. (19) and the numerical results obtained by Radjai et al. [200] on the probability distribution of contact forces, Ancey et al. showed that the bulk friction angle was weakly dependent on the particle friction coefficient [14]. Other effects such as the particle arrangement and the probability distribution of contact forces have greater influence on bulk friction. The result is in line with micromechanical analysis done in soil mechanics $[52,53,97]$.

For cases when particle contact is lubricated by the interstitial fluid, a number of theoretical models based on Eq. (19) have been proposed to compute the bulk stress tensor $[3,40,92,95]$. These models predict a viscous behavior, with no yield stress, but a diverging bulk viscosity when the solid concentration tends toward the maximum solid concentration. Indeed, the squeezing force between two neighboring particles is $\mathbf{F}=-3 \mu \pi a^{2} \mathbf{v} /(8 s)$, with $\mathbf{v}$ the relative particle velocity and $s$ the mean distance between the particle surfaces [92]; when the solid concentration is increased, the particles are more densely packed, which leads to decreasing $s$. However, the prediction of the nonexistence of a yield stress contrasts with experimental observation. Clear evidence of yielding behavior has been reported by Husband et al. [122] (with polyisobutylene/calcium carbonate suspensions). They observed that for solid concentrations in excess of a critical value ( $\phi \approx 0.47)$, suspensions exhibited a yield stress. Moreover, this yield stress increased dramatically when the solid concentration came closer to the maximum concentration. In this case, the authors attributed yielding behavior to either particle jams or weak polymer-particle interactions, but they did not provide quantitative justification in their explanations. Such 
behavior was also observed by Wildemuth and Williams [238] with coal-glycerin slurries, Kytömaa and Prasad [144,197] with $2 \mathrm{~mm}$ glass beads in a water-glycerol solution, Coussot [65] with $100 \mu \mathrm{m}$ polystyrene beads in water-glycerol solutions, and Johma et al. [134] with $2 \mu \mathrm{m}$ polystyrene beads in water. In the latter case, the authors related the yield appearance to structural changes in the particle arrangement (glass transition) at a critical solid concentration $(\phi=0.58)$. Using polymethylmethacrylate spheres in a Newtonian fluid, Heymann et al. found that their suspensions exhibited an apparent yield stress [108]. Surprisingly enough, the dependencies of this yield stress and the relative viscosity on the solid concentration revealed a similar trend. They also observed that there was an elastic-viscous transition separating the solid-like and fluid-like domains, implying that there is not a single yield stress. Wildemuth and Williams [238] have suggested that the existence of a yield stress in noninteracting particle suspensions is a consequence of a dependence of the maximum solid concentration on the shear stress. Using heuristic arguments, they have shown that a yield stress should arise over a given range of solid concentrations $\left[\phi_{0}, \phi_{\infty}\right]$ :

$\tau_{c}(\phi)=\sqrt[m]{A\left(\frac{\phi / \phi_{0}-1}{1-\phi / \phi_{\infty}}\right)}$

where $A, \phi_{0}$, and $\phi_{\infty}$ are three parameters. $\phi_{\infty}$ is the high-shear limit of the solid concentration and $\phi_{0}$ corresponds to a kind of percolation threshold. The model has been successfully tested by Wildemuth and Williams on coal slurries. Experiments conducted on suspensions with solid concentrations $\phi \leq 0.51$ have revealed a complex behavior (thixotropy due to order/disorder transition), but no yield stress [235]. These experiments substantiate the idea that there is a critical solid concentration (or narrow range of solid concentrations) $\phi_{\mathrm{c}}$, for which a percolating network of particles develops throughout the bulk.

\subsection{Constitutive equations: behavior at higher shear rates}

In principle, using the theoretical framework depicted in Section 2.2 makes it possible to compute the bulk stress tensor for particle suspensions. Rigorous analytical results have been obtained only for certain flow conditions (e.g., when $R e_{\mathrm{p}}$ and $S t$ are much smaller than unity) and dilute suspensions $[36,38,111,146,162,206,245]$. In contrast, for moderate and concentrated suspensions or for general flow conditions (i.e., the Stokes, Péclet, and Reynolds numbers taking any finite value), there is no full analytical derivation of the bulk stress tensor, but only approximate models based on heuristical simplifications or numerical simulations [14,244,246]. To progress in determining the rheological properties of particle suspensions, the basic idea is to look for prevailing terms in Eqs. (13) and (14) depending on the flow conditions. This may be done typically using dimensional analysis. For an interaction to be predominant, it must have (i) sufficient strength relative to others and (ii) time for its effects to influence the system. In practice, most of the dimen-

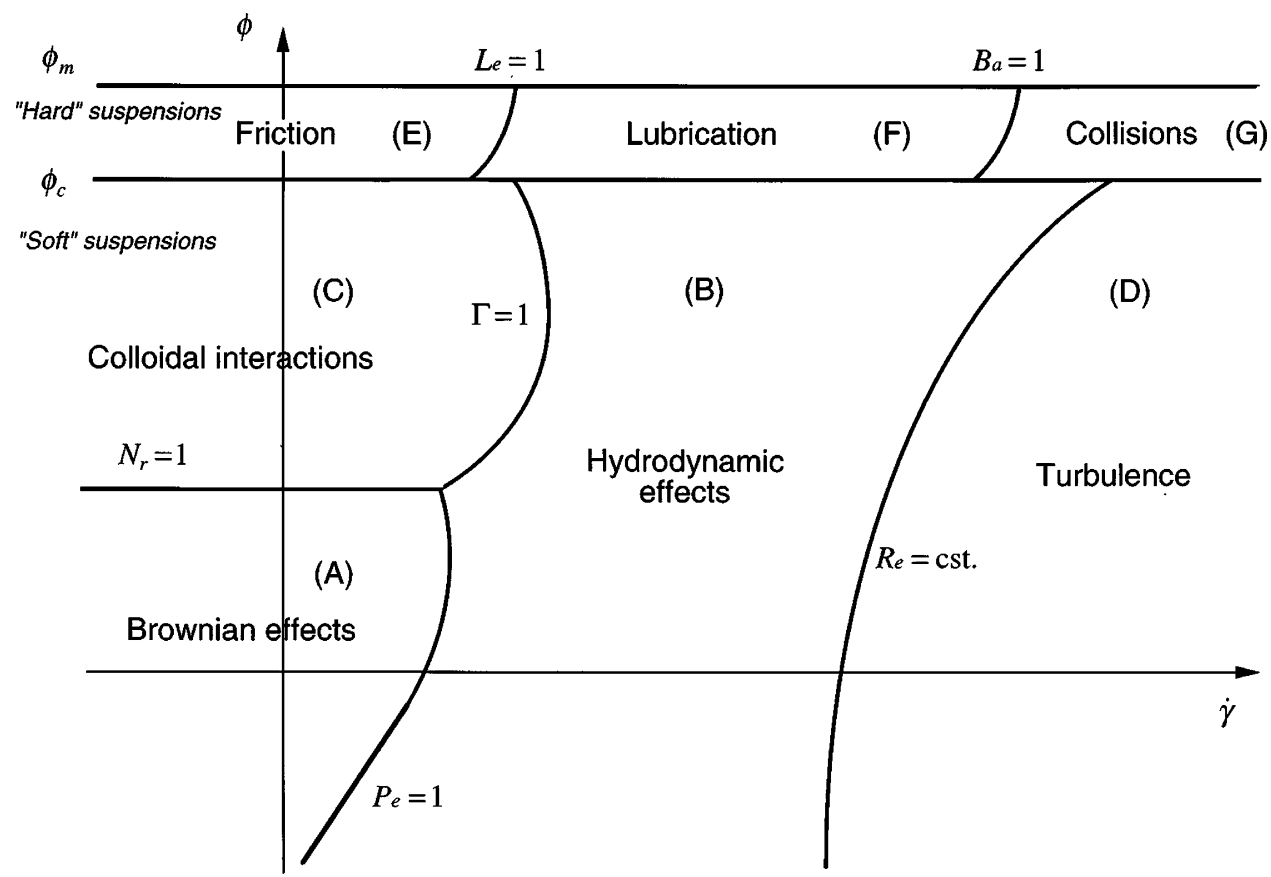

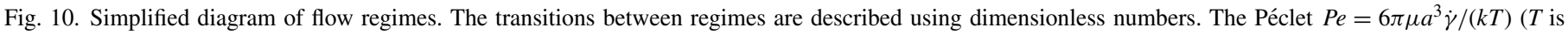

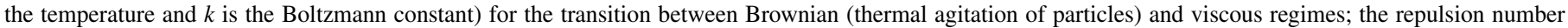

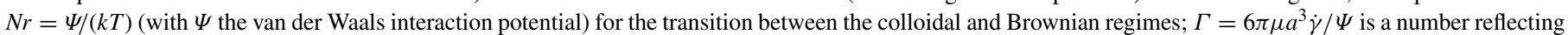

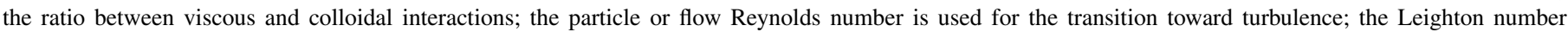

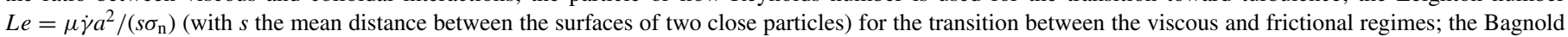

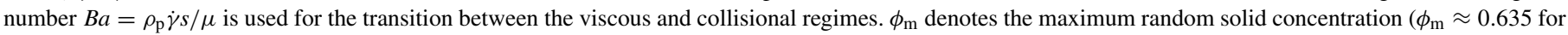

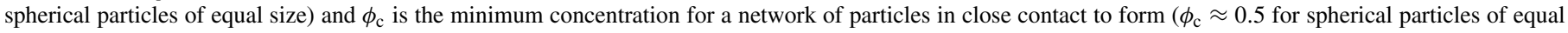
size). 
sionless numbers can be interpreted in this way. For instance, the Stokes number can be seen as the ratio of particle/fluid relaxation times or the ratio of inertia/viscous effects. Using a limited number of dimensionless numbers makes it possible to outline the flow regimes in a single diagram $(\dot{\gamma}, \phi)$ where $\dot{\gamma}$ is the shear rate (see. Fig. 10), as suggested by Coussot and Ancey [66].

For the hydrodynamic regime (B in Fig. 10), theoretical models predict a pseudo-Newtonian behavior, with a bulk viscosity $\eta$ rising with increasing solid concentration $\phi$ and diverging when the solid concentration comes closer to the maximum solid concentration $\phi_{\mathrm{m}}$. In these models, the dependence of $\eta$ on $\phi$ is similar to that given by Krieger and Dougherty's expression

$\eta=\mu\left(1-\frac{\phi}{\phi_{\mathrm{m}}}\right)^{-[\eta] \phi_{\mathrm{m}}}$

where $[\eta]=\lim _{\phi \rightarrow 0}(\eta-\mu) /(\mu \phi)=2.5$ is called the intrinsic viscosity. This type of relation matches the Einstein expression at low solid fractions. The divergence of the bulk viscosity when $\phi \rightarrow \phi_{\mathrm{m}}$ is not realistic from a physical point of view. In fact, beyond a critical value of $\phi$, colloidal interactions or direct/lubricated contacts may become predominant. Concentrated suspensions $(\phi>0.3)$ usually exhibit normal-stress effects, partly because of particle migration in simple shear experiments [243] or asymmetric microstructure [187].

The transition between the hydrodynamic regime (B in Fig. $10)$ and the colloidal regime ( $\mathrm{C}$ in Fig. 10) are of great interest for the applications since they correspond to viscoplastic behavior. To date, there is, however, no theoretical derivation, even approximate, of the bulk stress tensor. Phenomenological laws are therefore used to describe rheological behavior. One of the most popular is the Herschel-Bulkley model, which generalizes the Bingham law

$\tau=\tau_{\mathrm{c}}+K \dot{\gamma}^{n}$,

with $K$ and $n$ two constitutive parameters. In practice, this phenomenological expression successfully describes the rheological behavior of many materials over a sufficiently wide range of shear rates $[44,65]$, except at very low shear rates [71,94]. For numerical purposes, a viscoplastic model may be regularized using a biviscous model [83,239], Papanastasiou's exponential model [186], or extended forms [248]. Indeed, the existence of a yield stress entails numerical difficulties in tracking the shape and position of the yield surface(s) within the flow.

At high solid concentrations (regimes E-G in Fig. 10), there is a significant change in bulk behavior due to the development of a particle network within the bulk. A number of striking phenomena (dilatancy, jamming, shear localization, etc.) are induced by this network. Usually three subdomains can be considered: direct friction (regime E), lubricated contact (regime F), and collisional contact (regime G).

Regime E corresponds to the post-failure domain in soil mechanics, i.e., when after yielding, a soil creeps. As explained in Section 2.3, Coulomb friction at the particle level imparts its key properties to the bulk, which explains (i) the linear relationship between the shear stress $\tau$ and the effective normal stress $\sigma^{\prime}=\sigma-p$ (with $p$ the interstitial pore pressure)

$\tau=\sigma^{\prime} \tan \varphi$,

and (ii) the non-dependence of the shear stress on the shear rate $\dot{\gamma}$.

Regime F (lubricated contact, also called the macro-viscous regime by Bagnold [19]) may be seen as a mere extension of the hydrodynamic regime (B) since the bulk rheological behavior is still governed by the interstitial fluid. There is, however, a significant departure from Newtonian behavior when $\phi \rightarrow \phi_{\mathrm{m}}$. Indeed, the shear-induced relative motion of particle layers develops normal forces: a particle in motion can no longer travel far away from neighboring particles, but must slide between the particles of the surrounding layers (above and below it). The particle configuration is no longer isotropic and constant; crowding effects induce some organization or disorder depending on the shear rate [99]. For uniform hard-sphere suspensions, shear-thickening behavior appears for $\phi \geq \phi_{\mathrm{c}}$ because of order/disorder transitions or cluster formation [27,47,112,113,235].

Regime $\mathrm{G}$ (collisional contacts, also called the particle-inertia regime by Bagnold [19]) has long been characterized using kinetic theory or Bagnold-like heuristical arguments. [98,202]. For the same reason as for regime $\mathrm{F}$, there are significant differences between dilute and dense collisional regimes when the solid concentration exceeds a critical value $\phi_{\mathrm{c}}$. For $\phi>\phi_{\mathrm{c}}$, the development of a particle network together with the increasing contribution of frictional dissipation modify the structure of the bulk stress tensor. This regime is sometimes called frictionalcollisional to emphasize the importance of friction. The first proposition of bulk stress tensor seems to be attributable to Savage [210], who split the shear stress into frictional and collisional contributions

$\tau=\sigma \tan \varphi+\mu(T) \dot{\gamma}$,

with $T$ the granular temperature. Elaborating on this model, Ancey and Evesque suggested that there is a coupling between frictional and collisional processes [10]. Using heuristic arguments on energy balance, they arrived at the conclusion that the collisional viscosity should depend on the Coulomb number Co $=\rho_{\mathrm{p}} a^{2} \dot{\gamma}^{2} / \sigma$ to allow for this coupling in a simple way

$\tau=\sigma \tan \varphi+\mu(\mathrm{Co}) \dot{\gamma}$.

Pouliquen et al. proposed a slightly different version of this model, where both the bulk frictional and collisional contributions collapse into a single term, which is a function of the Coulomb number $[93,136,195]$

$\tau=\sigma \tan \varphi(\mathrm{Co})$.

Contrasting with other propositions, Josserand et al. stated that the key variable in shear stress was the solid concentration $\phi$ rather than the Coulomb number [137]

$\tau=K(\phi) \sigma+\mu(\phi) \dot{\gamma}^{2}$,

with $K$ a friction coefficient. Every model is successful in predicting experimental observations for some flow conditions, but to date, none is able to describe the frictional-collisional regime for a wide range of flow conditions and material properties. 


\subsection{The case of polydisperse suspensions}

Natural suspensions are made up of a great diversity of grains and fluids. This observation motivates fundamental questions: how to distinguish between the solid and fluid phases? What is the effect of colloidal particles in a suspension composed of coarse and fine particles? We shall see that, when the particle size distribution is bimodal (i.e. we can distinguish between fine and coarse particles), the fine fraction and the interstitial fluid form a viscoplastic fluid embedding the coarse particles, as suggested by Sengun and Probstein [218]. This approximation usually breaks for poorly sorted slurries. In that case, following Iverson [128,129], we will see that Coulomb plasticity can help understand the complex, time-dependent rheological behavior of slurries.

Sengun and Probstein proposed different arguments to explain the viscoplastic behavior observed in their investigations on the viscosity of coal slurries (with particle size typically ranging from 0.4 to $300 \mu \mathrm{m}$ ) [218,219]. Their explanation consists of two approximations. First, as this is the interstitial phase, the dispersion resulting from the mixing of fine colloidal particles and water imparts most of its rheological properties to the entire suspension. Secondly, the coarse fraction is assumed to act independently of the fine fraction and to enhance bulk viscosity. They introduced a net viscosity $\eta_{\mathrm{nr}}$ of a bimodal slurry as the product of the fine relative viscosity $\eta_{\mathrm{fr}}$ and the coarse relative viscosity $\eta_{\mathrm{cr}}$. The fine relative viscosity is defined as the ratio of the apparent viscosity $\eta_{\mathrm{f}}$ of the fine-particle suspension to the viscosity of the interstitial fluid $\mu: \eta_{\mathrm{fr}}=\eta_{\mathrm{f}} / \mu$. The coarse relative viscosity is defined as the ratio of the apparent viscosity $\eta_{\mathrm{c}}$ of the coarseparticle slurry to the viscosity of the fine-particle suspension: $\eta_{\mathrm{cr}}=\eta_{\mathrm{c}} / \eta_{\mathrm{f}}$. The two relative viscosities depend on the solid concentrations and a series of generalized Péclet numbers. For the coarse-particle suspensions, all the generalized Péclet numbers are much greater than unity. Using a dimensional analysis, Sengun and Probstein deduced that the coarse relative viscosity cannot depend on the shear rate. In contrast, bulk behavior in fine-particle suspensions is governed by colloidal particles and thus at least one of the generalized Péclet numbers is of the order of unity, implying that the fine relative viscosity is sheardependent. Sengun and Probstein's experiments on the viscosity of coal slurries confirmed the reliability of this concept [218]. Plotting $\log \eta_{\text {nr }}$ and $\log \eta_{\text {fr }}$ against $\log \dot{\gamma}$, they found that over a wide range of concentrations, the curves were parallel and their distance was equal to $\log \eta_{\mathrm{cr}}$ (see Fig. 11). However, for solid concentrations in the coarse fraction exceeding 0.35 , they observed a significant departure from parallelism which they ascribed to nonuniformity in the shear rate distribution within the bulk due to squeezing effects between coarse particles.

Ancey and Jorrot examined the effect of adding coarse particles in a colloidal dispersion [11]. At first glance, since the volume occupied by the colloidal particles is decreased, the bulk yield stress should decrease and, to first order, we can use Eq. (20) to infer

$\tau_{\mathrm{c}}=\frac{K}{(2 a)^{2}}\left(\frac{\phi_{\mathrm{f}}}{1-\phi_{\mathrm{f}}}\right)^{c}(1-\phi)$,

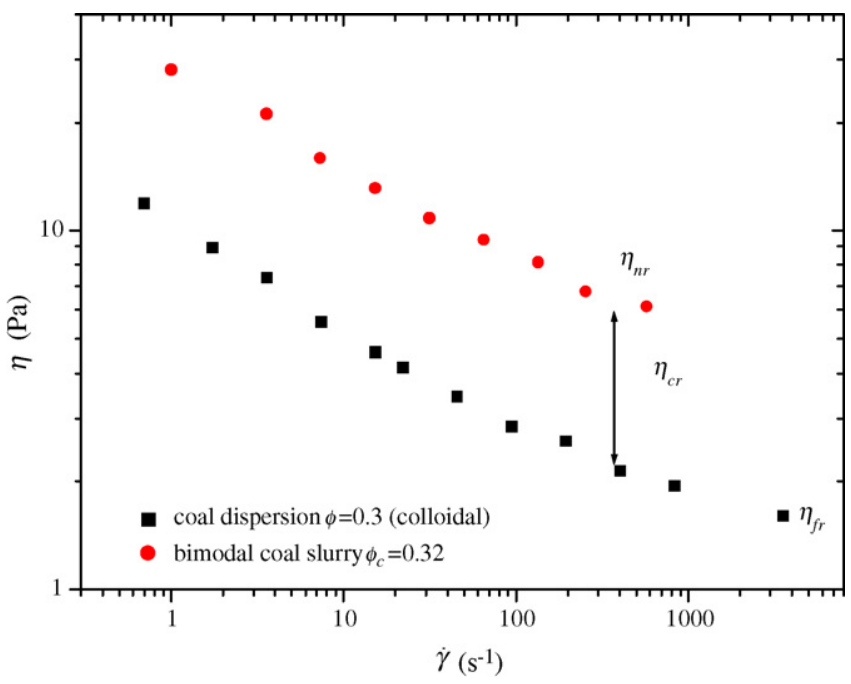

Fig. 11. Variation in the bulk viscosity of coal slurry as a function of the shear rate. The bulk viscosity curve is parallel to the curve obtained with the fine fraction. After [218].

where $\phi$ is the coarse-particle concentration and $\phi_{\mathrm{f}}$ the concentration in fine (colloidal) particles. To test this expectation, Ancey and Jorrot measured the bulk yield stress of kaolin suspensions to which they added a given amount of coarse particles. Fig. 12 shows typical results obtained with a bimodal distribution of glass beads ( 1 and $3 \mathrm{~mm}$ in diameter). The dimensionless number $\xi$ is the relative fraction of small beads $(\xi=0$ means that there were no small beads while $\xi=1$ means that all coarse particles added to the kaolin suspension were small beads). The total solid concentration $\phi_{\mathrm{t}}$ is computed as follows: $\phi_{\mathrm{t}}=\phi_{k}\left(1-\phi_{\mathrm{c}}\right)+\phi_{\mathrm{c}}$. The first result is that the trend given by Eq. (20) is correct to first order: adding a small amount of coarse particles leads to a decrease in the bulk yield stress (here for total solid concentrations as high as 0.55 ). Interestingly enough, in contrast with the authors' expectation, the bulk yield stress starts diverging when the total solid concentration comes closer to the maximum solid concentration. A striking feature of this abrupt rise is that the increase rate is very close to the value measured for a pure kaolin dispersion. This could mean that coarse particles surrounded by

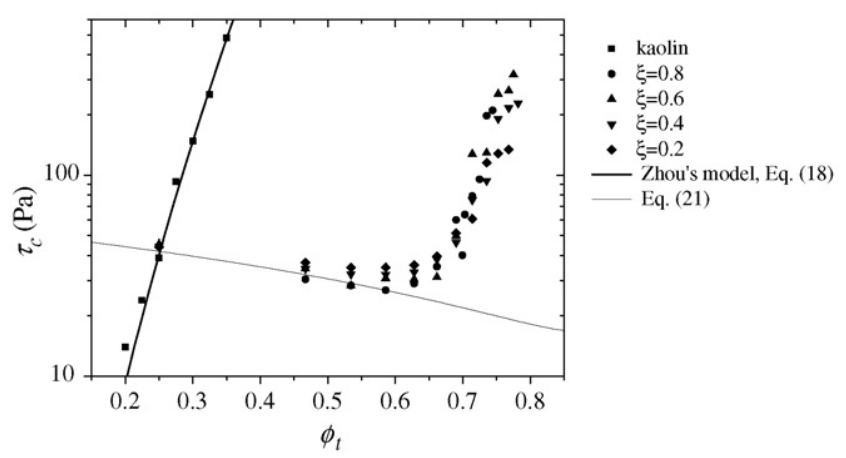

Fig. 12. Variation in the bulk yield stress. The variation in the yield stress for a kaolin suspension is reported as a function of the solid concentration ( $\phi_{\mathrm{t}}$ coincides with the fine fraction). The thin solid line represents the expectation of a decreasing bulk yield stress with increasing coarse concentration (see Eq. (23)). The symbols represent the experimental data obtained by varying the ratio $\xi$ of large and small beads. After [11] 
colloidal particles may very well behave in turn as colloidal particles (this statement is naturally wrong). Further comments on Fig. 12 are the following:

- At low and moderate concentrations of coarse particles, the bulk yield stress was independent of the particle size (when equal size distributions were tested), but it increased significantly with increasing relative fractions of large particles.

- On the contrary, at high concentrations, the finer the distribution, the larger the yield stress.

The main and unexpected result of this experimental study is that bulk yield stress may be significantly affected by the concentration of coarse particles, but its features (such as the growth rate with a solid concentration) are still governed by the fine colloidal fraction.

Given substantial experimental difficulties (particle size, sedimentation, etc.), few experimental investigations have been conducted on poorly-sorted slurries. In soil mechanics, testing bulk materials in quasi-static drained or non-drained flow configurations has shown that shear strength is governed by compaction state and pore fluid pressure [78,234]. Since geotechnical tests can hardly be run under large deformations, Iverson and his colleagues carried out experiments in a $95 \mathrm{~m}$ long flume, specifically built in Oregon (USGS flume) [131]. In Iverson's opinion, the flow of poorly sorted mixtures is fundamentally an unsteady phenomenon, which cannot be easily investigated under steady flow conditions. Indeed, the shear strength adheres to the Coulomb law: $\tau=\sigma^{\prime} \tan \varphi$, with $\sigma^{\prime}=\sigma-p$ the effective stress. During the motion, the material contracts, which gives rise to high pore pressure and thus a decrease in shear stress. Pore pressure can remain elevated when pore pressure diffusion is slow (i.e., for low bulk permeability), as shown by Eq. (6). Consequently, shear strength is not a rheological property [127].

Is it possible to provide clear evidence for the prevalence of Coulomb frictional behavior and dependence of shear strength on pore pressure in rapidly sheared, poorly sorted slurries? Because of the unsteady nature of shear strength together with the number of control variables that are also time-dependent (pore pressure, solid concentration, normal stress), providing an indisputable reply to this question remains difficult. There are, however, a number of laboratory and field observations that support this theory. For instance, carrying out experiments with poorly sorted materials in the USGS flume, Major observed that increasing the fine fraction resulted in thinning the deposit layer, which meant that the bulk strength decreased [156] (see also [127]). This observation conflicts with laboratory experiments showing an increase in yield stress when the fine fraction is increased (see the asymptotic trend in Fig. 12 when $\phi_{\mathrm{t}} \rightarrow \phi_{\mathrm{m}}$ ), but can be explained by recognizing that increasing the fine content leads to a decrease in the bulk permeability and consequently reduces pore pressure diffusion; the bulk stays longer in a liquified state, with high pore-pressure levels and low shear strength. In the next section, we will present laboratory experiments that also provide support for this explanation.

\section{Rheometrical experiments}

Over the last 20 years, a large number of experiments have been carried out to test the rheological properties of natural materials. The crux of the difficulty lies in the design of specific rheometers compatible with the relatively large size of particles involved in geophysical flows. Coaxial-cylinder (Couette) rheometers and inclined flumes are the most popular geometries. Another source of trouble stems from disturbing effects such as particle migration and segregation, flow heterogeneities, fracturation, layering, etc. These effects are often very pronounced with natural materials, which may explain the poor reproducibility of rheometrical investigations $[62,126,158]$. Poor reproducibility, complexity in the material response, and data scattering have at times been interpreted as the failure of the onephase approximation for describing rheological properties [126] . In fact, these experimental problems demonstrate above all that the bulk behavior of natural material is characterized by wide fluctuations, which can be as wide as the mean values. As for turbulence and Brownian motion, we should describe not only the mean behavior, but also the fluctuating behavior to properly characterize the rheological properties. For concentrated colloidal or granular materials $[54,107,152,175,181,222,231]$, experiments on well-controlled materials have provided evidence that to some extent, these fluctuations originate from jamming in the particle network (creation of force vaults sustaining normal stress and resisting against shear stress, both of which suddenly relax). Other processes such as ordering, aging, and chemical alteration occur in natural slurries, which may explain their timedependent properties $[39,163]$. Finally, there are disturbing effects (e.g., slipping along the smooth surfaces of a rheometer), which may bias measurement.

Table 1 reports a number of experimental investigations run on natural samples collected in the field or materials mimicking natural materials. The list is far from exhaustive. For Coulomb plastic materials, apart from experimental tests conducted by Savage, Hutter, and Iverson et al., which are cited in Table 1 , most authors have tried to document that shear stress depends on the solid concentration or the shear rate, as expected from kinetic theory or Bagnold-like phenomenological laws $[15,17,87,177,178,195,228,232]$. These authors are not cited in Table 1.

Here, we will not examine at length the various experiments supporting either the viscoplastic or the Coulomb plastic model, but we will try to understand in which conditions a material can behave like a viscoplastic fluid. This analysis is mostly based on the rheometrical investigation carried out by Ancey with a Couette cell [6]. We will then examine the consequences of the rheological properties on the flow features. This analysis will rely on the flume experiments conducted by Parsons et al. [188] and Iverson et al. [127-129,131,156].

\subsection{Couette-cell experiments on granular mixtures}

In order to study the influence of lubricated contact on bulk dynamics and provide evidence of the key role played by the particle network in the rheological properties of highly con- 
Table 1

Experimental investigations conducted on natural materials or nearly natural materials

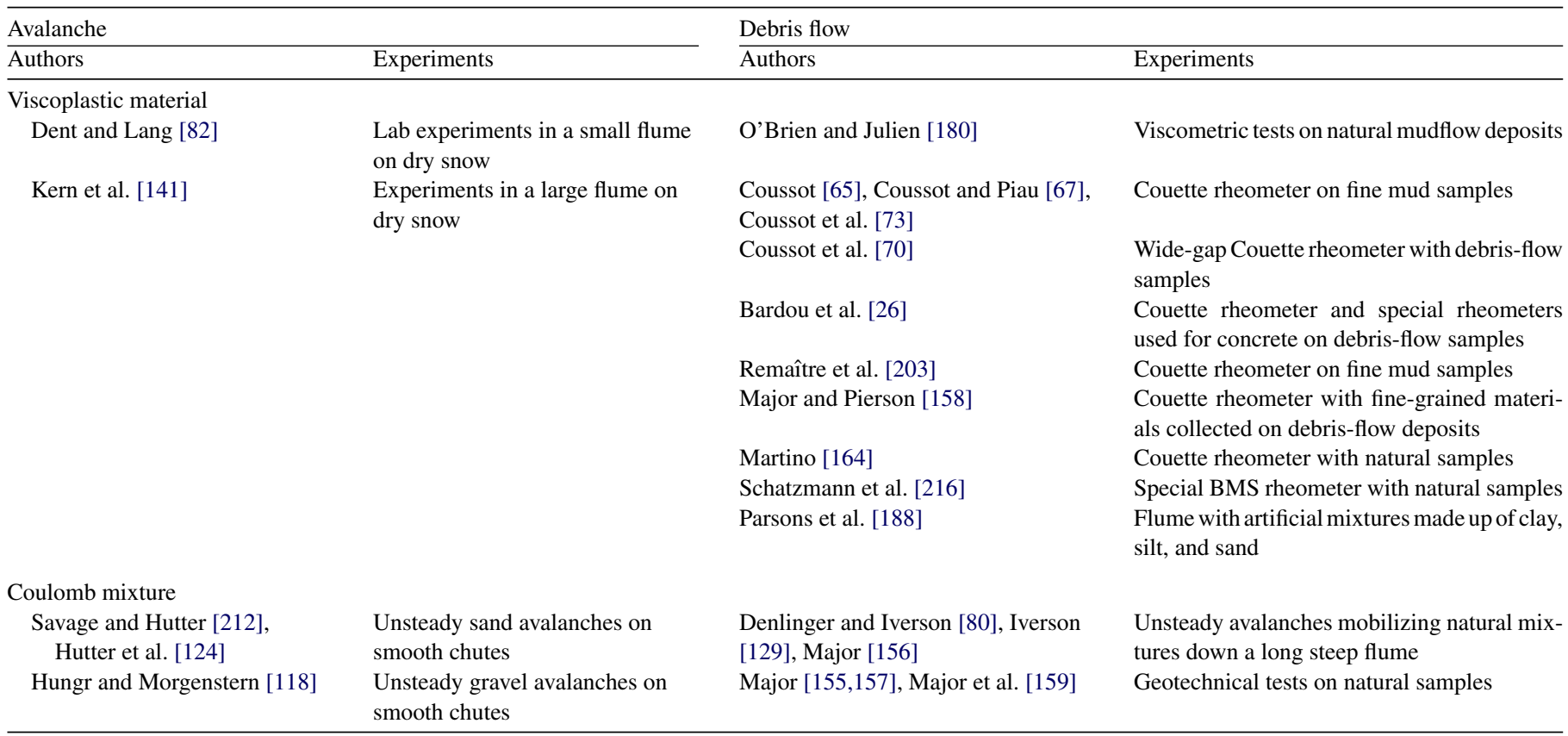

centrated suspensions, Ancey studied a number of suspensions made up of glass beads and various interstitial fluids: air $(\mu=$ $\left.1.8 \times 10^{-5} \mathrm{~Pa} \mathrm{~s}\right)$, water $\left(\mu=10^{-3} \mathrm{~Pa} \mathrm{~s}\right)$, a water-glycerol solution $\left(\mu=0.96 \mathrm{~Pa} \mathrm{~s}, \rho_{\mathrm{f}}=1260 \mathrm{~kg} / \mathrm{m}^{3}\right)$, and a water-kaolin dispersion [6]. The particle diameter was $0.3,0.8,1,2$, or $3 \mathrm{~mm}$. For the rheometrical tests, Ancey used a Haake Rotovisco rheometer with a four-blade vane centered around a vertical shaft. This technique from soil mechanics is now increasingly used in rheometry of suspensions [30]. The radius of the vane was $R_{1}=30 \mathrm{~mm}$. The solid concentration in coarse particles $\phi$ was very close to the maximum concentration $\phi_{\mathrm{m}}$ (here we have $\phi=0.58-0.61$ while $\phi_{\mathrm{m}}=0.635$ ).

Fig. 13 shows the variation in the dimensionless shear stress $S=\tau /(\bar{\rho} g h)$ (where $\tau$ denotes the shear stress and $h$ is the thickness of material sheared by the vane) as a function of a dimensionless number $\Gamma=\mu \Omega /\left(\bar{\rho}^{\prime} g h\right)$ (where $\Omega$ is the rotational speed of the vane, $\bar{\rho}^{\prime}=\bar{\rho}-\rho_{\mathrm{f}}$ is the buoyant density, $\mu$ the viscosity of the interstitial fluid). $\Gamma$ is a dimensionless shear rate. Ancey replaced the true shear rate by the rotational speed because determining the actual shear rate for a large-gap rheometer and a material with varying rheological properties is very delicate (see below). Let us note that this number is very close to the Leighton number introduced in the caption of Fig. 10 or the friction number introduced by Iverson [129]. Although the experimental curve reported in Fig. 13 does not provide the proper flow curve, it can provide an approximate idea of this flow curve. Two trends can be observed

- At low rotational velocities $(\Gamma \ll 1)$, shear was localized within a narrow cylindrical band around the vane, with a typical thickness of approximately 10 bead diameters independently of $\Gamma$. Ancey found that $S$ was independent of $\Gamma$ which implies, when one returns to dimensional variables, that: (i) $\tau \propto \sigma_{z z}$ (where $\sigma_{z z}$ denotes the vertical normal stress) and (ii) $\tau$ does not depend on the shear rate, but is linear with the vertical normal stress. Both features are typical of the

Table 2

Features of materials used and flow conditions for experimental run reported in Figs. 13-15

\begin{tabular}{|c|c|c|c|c|c|c|c|c|}
\hline Class & Material & $\phi_{\mathrm{f}}$ & $\phi_{\mathrm{t}}$ & $\phi$ & $N$ & $\tau_{\mathrm{c}}$ & $h$ & Fluid \\
\hline \multirow[t]{6}{*}{ Granular suspensions } & Material E0 & 0 & 61.3 & 61.3 & - & 0 & 43 & Water \\
\hline & Material E1 & 0 & 61 & 61 & - & 0 & 44 & Air \\
\hline & Material E2 & 0 & 61 & 61 & - & 0 & 14 & Water \\
\hline & Material E4 & 0 & 60 & 60 & - & 0 & 21 & Glycerol \\
\hline & Material E5 & 0 & 60 & 60 & - & 0 & 32 & Glycerol \\
\hline & Material E6 & 2 & 60.9 & 61.6 & 12.4 & 0.2 & 34 & Water \\
\hline Class 3 & Material C & 15.4 & 47.9 & 55.9 & 0.3 & 4.5 & 60 & Water \\
\hline
\end{tabular}

Solid concentrations are in \%, flow depth $h$ in mm, yield stress $\tau_{\mathrm{c}}$ in Pa. 


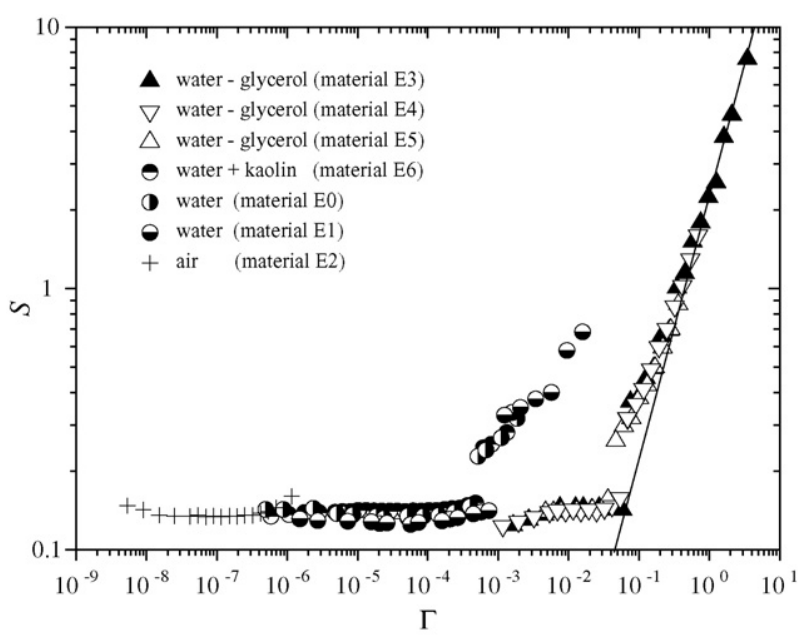

Fig. 13. Variation in the dimensionless shear stress as a function of the dimensionless number $\Gamma$. The line slope is unity and indicates a linear variation of $S$ with $\Gamma$. The features of each material are specified in Table 2. After [6,9].

frictional regime. The relation $\tau \propto \sigma_{z z}$ implies that the total torque should be a quadratic function of the flow depth for a Coulomb material, as is shown in Fig. 14.

- At high rotational velocities, all the material was sheared in the gap. $S \propto \Gamma$, that is, in terms of dimensional variables, $\tau \propto \dot{\gamma}$. The bulk behavior is similar to that of a Newtonian fluid for these flow conditions.

A striking result of this experiment is that it is possible to observe very different bulk rheological behavior by merely increasing the shear rate and keeping the solid concentration fairly constant. A difficult point in the rheometrical analysis is the derivation of the flow curve. Indeed, in a Couette cell, the shear rate is found by solving the following equation

$\Omega=\int_{R_{1}}^{R_{2}} \frac{\dot{\gamma}(r)}{r} \mathrm{~d} r$,

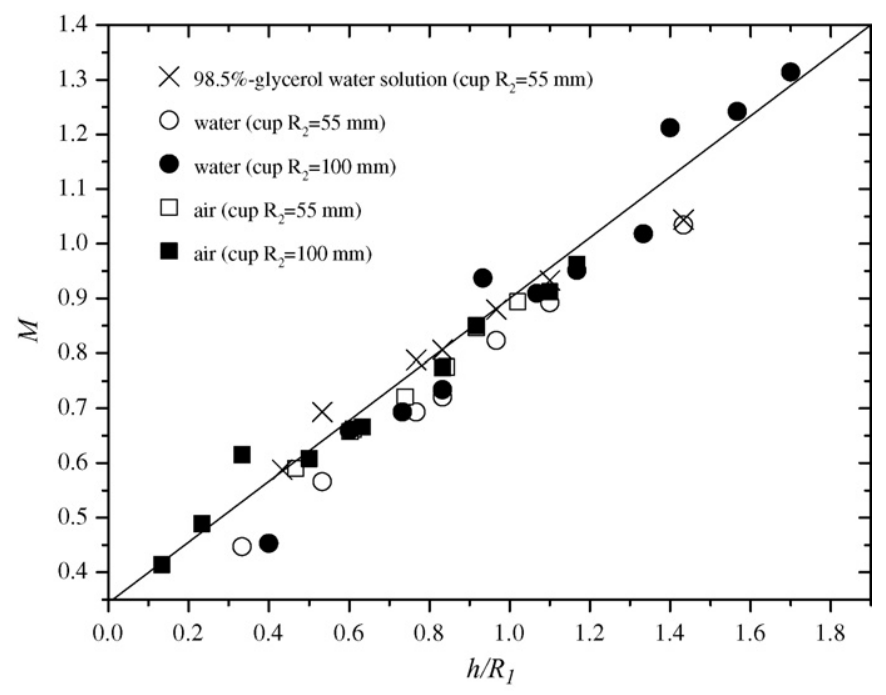

Fig. 14. Variation in the reduced torque $M=C /\left(\pi \bar{\rho}^{\prime} g h R_{1}^{2}\right)$ as a function of the scaled flow depth $h / R_{1}$, with $R_{1}$ the inner cylinder radius. After $[6,9]$.

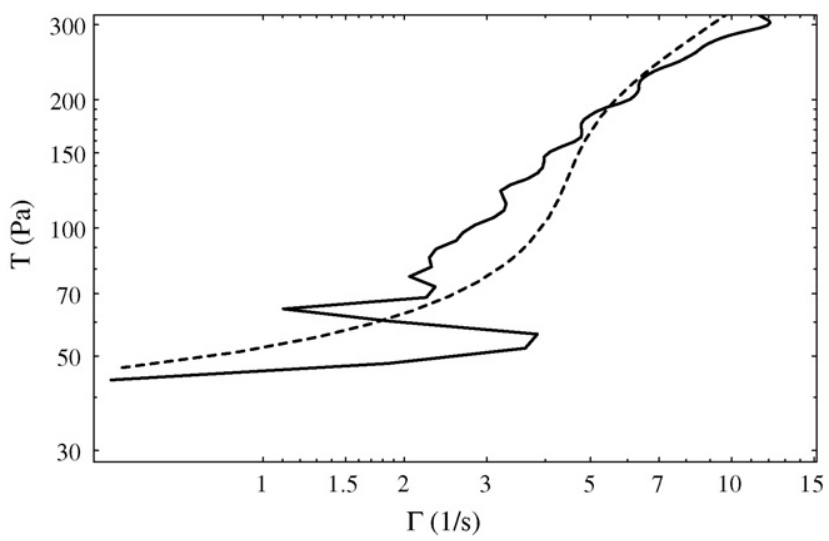

Fig. 15. Flow curve for $1 \mathrm{~mm}$ beads within a water-glycerol solution $(\phi=$ $0.605, \mu_{0}=1.05 \mathrm{Pas}$ ). The solid line represents the WVD solution, while the dashed line represents the Tikhonov solution. After [7].

where $R_{2}$ denotes the outer-cylinder radius. For thin-gap rheometers, Eq. (24) can be easily approximated to first order: $\Omega \approx\left(R_{2}-R_{1}\right) \gamma$. For wide-gap rheometers, specific techniques must be used, such as the Tikhonov regularization method [241]. They may, however, induce errors in smoothing out the flow curve when this curve undergoes abrupt changes (e.g., transition from frictional to viscous regimes). To solve Eq. (24), Ancey developed an alternative method called wavelet-vaguelette decomposition (WVD), which is based on wavelets and projection methods [7] (Table 2).

The outcomes of the WVD and Tikhonov methods are reported in Fig. 15. In this figure, the shear rate was computed by solving Eq. (24); note that the resulting shear rate is equivalent to a shear rate that would have been measured at the inner-cylinder boundary. Taking a closer look at the WVD solution, we observe that, for low shear stresses $(\tau<50 \mathrm{~Pa})$, the flow curve is approximately horizontal for shear rates in the range $0.1-4 \mathrm{~s}^{-1}$. At $\dot{\gamma}=4 \mathrm{~s}^{-1}$, a slight increase in the shear stress leads to a substantial decrease in the shear rate, which drops to $1 \mathrm{~s}^{-1}$; this value is much higher than the value of $\dot{\gamma}_{\mathrm{m}}$, but this is normal since $\dot{\gamma}_{\mathrm{m}}$ has been estimated by assuming a sudden expansion of the sheared zone. For higher shear stress $(\tau>80 \mathrm{~Pa})$, the shear stress varies almost linearly with increasing shear rates. This result is consistent with our interpretation above. In contrast, the Tikhonov solution smooths the flow curve bulges, thus comparing well with the WVD solution only at very low and high shear rates $\left(\dot{\gamma}<1 \mathrm{~s}^{-1}\right.$ or $\left.>6 \mathrm{~s}^{-1}\right)$.

Ancey also studied poorly sorted suspensions by adding fine (kaolin) particles to a coarse-grained suspension [6]. The question was: how was bulk behavior affected by adding these particles? Experimental data are reported in Fig. 16, showing the torque exerted by the suspension on the vane as a function of its dimensionless rotational speed $\Gamma$. Obviously, when the solid concentration in fine particles $\phi_{\mathrm{f}}$ is low, there is not much difference compared to the results found above with the granular suspension (see Fig. 13). Conversely, when $\phi_{\mathrm{f}}$ is sufficiently high, bulk behavior is expected to be viscoplastic (Sengun and Probstein's approximation). Both statements are right, as shown in Fig. 16 (material A refers to a suspension poor in kaolin 


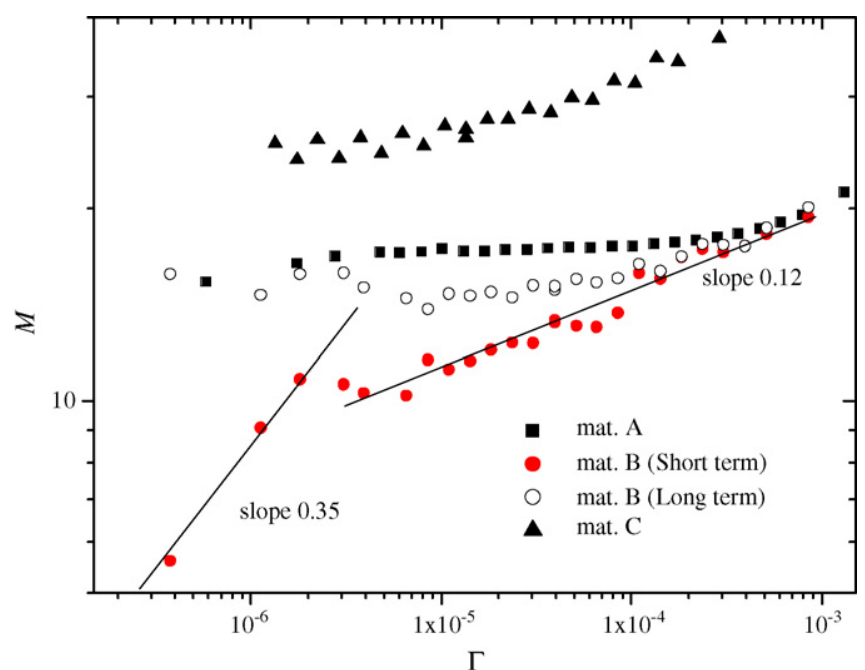

Fig. 16. Variation in the dimensionless torque $M=C /\left(\pi \rho^{\prime} g h R_{1}^{3}\right.$ ) (where $C$ is the measured torque) exerted on the vane by the tested suspension as a function of the rotational speed $\Gamma$. For material features, see Table 2. After [6].

while material $\mathrm{C}$ is rich in kaolin). At an intermediate concentration $\phi_{\mathrm{f}}$ (material B in Fig. 16), an odd behavior was observed.

Measuring the torque with time revealed that, when a shear rate was applied, the shear stress first increased rapidly and reached a maximum (short-term behavior), then decreased slowly and flattened out, and rose once again to finally attain its late-time value (typically after 1000 revolutions of the vane). Reporting the early-time and late-time values of the measured torque in Fig. 17, we observed a complicated response of the material: over a short time span, it behaved like a power-law (shearthinning) fluid while, over a long time span, its flow curve was identical to that of material A. A possible explanation for this behavior is that, when a shear rate step is applied, the network of particles is broken and contact between coarse particles is lubricated by the kaolin-water suspension. Since the yield stress of the kaolin-water suspension is not sufficient for coarse-particle

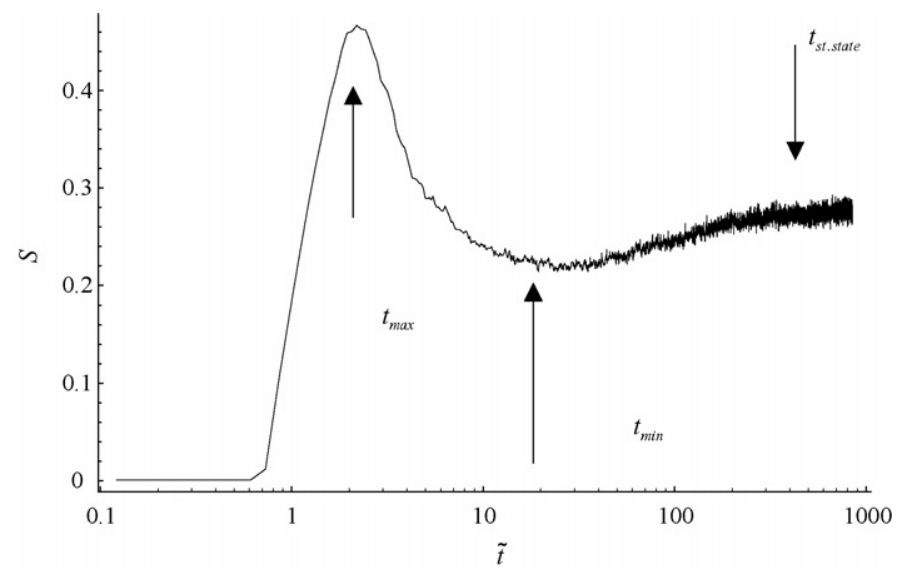

Fig. 17. Variation in the dimensionless shear stress $S=\tau /(\bar{\rho} g h)$ as a function of dimensionless time $\tilde{t}=t \Omega$. Experiments made for a suspension made up of glass beads $\left(\phi_{\mathrm{c}}=0.589, a=0.4 \mathrm{~mm}\right)$, kaolin $\left(\phi_{k}=0.098, \tau_{\mathrm{c}}=1.2 \mathrm{~Pa}\right)$, and water $\left(\phi_{\mathrm{t}}=0.629\right)$. After [6] sedimentation to be hindered, a network of particles in close contact forms again after a finite period of time. An alternative explanation is the following: according to Iverson [129], imposing a shear-rate step on the slurry first caused dilatancy at short times, then contraction within the bulk and a pore-pressure increase. Pore pressure slowly diffused (see Eq. (6)) until it became hydrostatic. According to the Coulomb law $(\tau=(\sigma-p) \tan \varphi)$, during the phase of contraction and high pore pressure, the shear stress was lower than its long-time value (when the pressure was became hydrostatic), but slowly increases toward this value as the pore pressure decreased.

A more quantitative analysis of Ancey's experiments can be performed as follows. Fine colloidal particles and water form a homogeneous colloidal blend, which becomes the interstitial fluid. If the yield stress of this blend is sufficiently high, it counterbalances settling effects for the coarse fraction. This explanation can be more evident using dimensional arguments. Let us consider two coarse neighboring particles within this blend. If these particles are squeezed to expel the thin layer of interstitial fluid between them, the normal-stress limit is $2 \tau_{\mathrm{c}}$. The squeezing force is the buoyant gravity force. We can define a dimensionless number $N$ as the ratio of a buoyancy stress (here $4 \rho^{\prime} g a / 3$ ) to the resisting force:

$N=\frac{2 \rho^{\prime} g a}{3 \tau_{\mathrm{c}}}$.

When $N \ll 1$, the blend impeded coarse-particle settling; as a result, the coarse particles cannot come into contact. From the rheological point of view, this entails that, since all the contacts are lubricated by a viscoplastic material, the bulk is in turn viscoplastic.

On the other hand, when $N \gg 1$, direct contacts between particles arise. For certain flow conditions (e.g., at low shear velocities $\Gamma \ll 1$ ), a percolating network of particles experiencing sustained frictional contacts develops, which means that the bulk behaves like a Coulomb mixture. Increasing the shear rate $(\Gamma \gg 1)$ can break direct contacts and induce contact lubrication.

Experimentally, three classes can be distinguished:

- For $N \geq 4$, the bulk behavior is either frictional $(\Gamma \ll 1)$ or viscous. The viscous behavior exhibits a shear-thinning trend.

- For $N \leq 1.1$, the bulk behavior is viscoplastic. This regime is quickly achieved (within a few milliseconds). For the same material and flow conditions, the flow curve varies significantly between two runs (deviation of the order of $\pm 10 \%$ ).

- For $1.1 \leq N \leq 4$, the bulk behavior depends on typical timescales. As shown by Fig. 17, when we impose a shearrate step, the mechanical response is time-dependent. A stress peak is first reached within a few milliseconds after the shear rate is imposed $\left(\tilde{t} \approx t_{\max }\right)$. The shear stress then relaxes and reaches a plateau. Finally, at long times (pour $\left.\tilde{t}>t_{\text {st.state }}\right)$, the shear stress increases and flattens out to reach a new plateau. For low shear rates $\left(\Gamma<10^{-5}\right)$, the characteristic times are nearly constant, with $t_{\max }=\mathcal{O}(2)$, $t_{\text {min }}=\mathcal{O}(10)$, and $t_{\text {st.state }}=\mathcal{O}(500)$. At high shear rates, the characteristics vary with $\Gamma: t_{\max } \propto \Gamma$, while $t_{\text {st.state }} \propto \Gamma^{-1}$. 


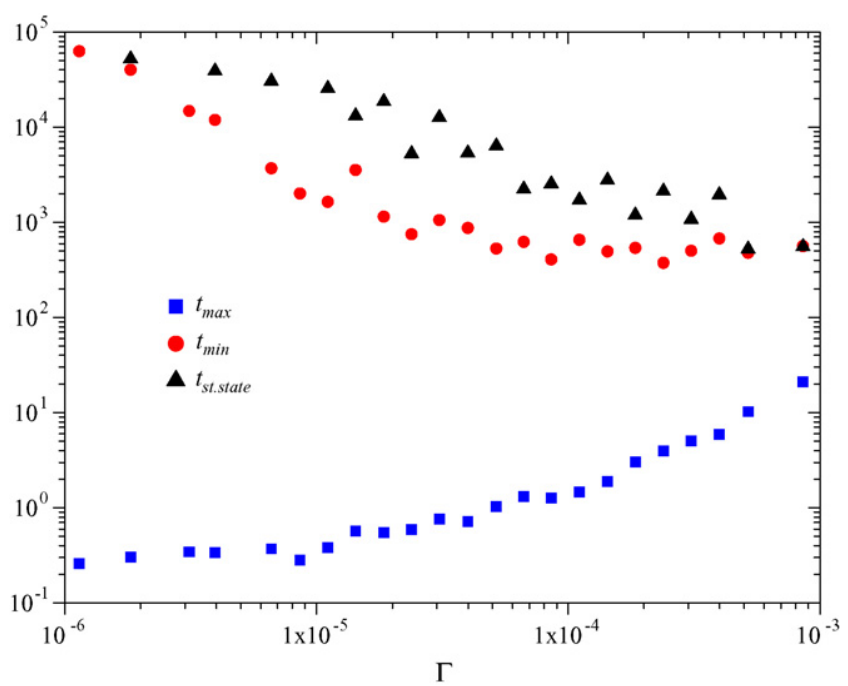

Fig. 18. Glass beads $\left(\phi_{\mathrm{c}}=0.589, a=0.4 \mathrm{~mm}\right)$, kaolin $\left(\phi_{k}=0.098, \tau_{\mathrm{c}}=\right.$ $1.2 \mathrm{~Pa})$, water $\left(\phi_{\mathrm{t}}=0.629\right)$. After [6].

It is worth noting that for $\Gamma \gg 1$, the timescales are similar: $t_{\text {max }} \simeq t_{\text {st.state }}=\mathcal{O}(100)$. It is then possible to distinguish an instantaneous behavior (at short times) and delayed behavior (long term, when a steady regime is achieved). The variation in the timescales with $\Gamma$ is reported in Fig. 18. The behavior for short times is shear thinning: $S \propto \Gamma^{0.35}$, whereas for long times, the bulk behavior is frictional.

\subsection{Flow features}

Parsons et al. ran a series of experiments to investigate the transition between viscoplasticity-dominated and frictiondominated regimes [188]. They used a semi-circular inclined flume and measured the velocity profile at the free surface; in addition, they estimated the bulk viscosity and yield stress using independent tests. Different slurries were prepared by altering the sand, clay, and silt fractions. They obtained muddy slurries, when the matrix was rich in silt and clay, and poorly sorted mixtures, when the silt and clay contents were reduced. Surprisingly enough, the change in fine-particle content did not significantly modify the appearance of the body, whereas it markedly altered the composition of the front and its behavior. In all the experiments, they found that the Herschel-Bulkley performed well since the velocity profile and the plug position were properly estimated. Reducing the fine fraction in the slurries induced a radical change of behavior for the front (see Fig. 19):

- For muddy slurries, the front takes the form of a blunt nose. Lack of slip along the flume bottom caused a conveyer-beltlike flow at the front.

- For coarse-grained slurries, the front takes the form of a dry granular locked nose slipping along the bed as a result of the driving force exerted by the fluid accumulating behind the snout. Additional material was gradually incorporated into the snout, which grew in size until it was able to slow down the body. (a)
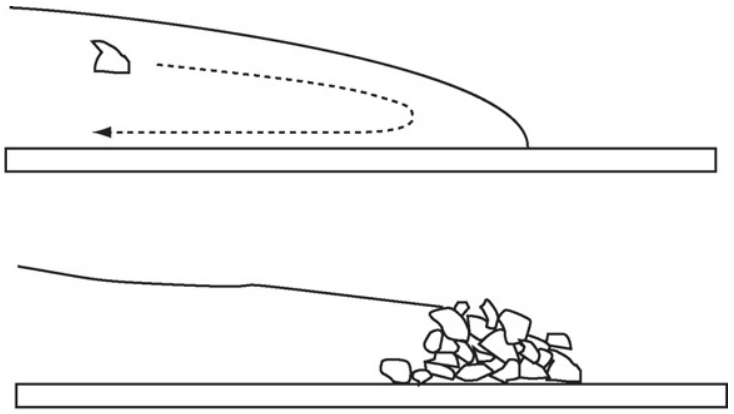

(b)

Fig. 19. Schematic of the behavior contrast beween fine-grained and coarsegrained flows. (a) Conveyer-belt-like flow at the front and (b) formation of a frictional front. After [188].

Interestingly enough, the changes in the rheological properties mainly affected the structure of the flow, especially within the tip region.

Iverson, Denlinger, and Major investigated slurries predominantly made up of a water-saturated mixture of sand and gravel, with a fine fraction of only a few percent [127-129,156]. Experiments were run on the USGS flume and consisted in releasing a volume of slurry (approximately $10 \mathrm{~m}^{3}$ ) down a $31^{\circ}, 95 \mathrm{~m}$-long flume. At the base of the flume, the material spread out on a planar, nearly horizontal, unconfined runout zone. Flow-depth, base normal stress, and base interstitial flow pressure were measured at different places along the flume. Iverson and his co-workers observed that at early times, an abrupt front formed at the head of the flow, followed by a gradually tapering body, then a thin, more watery tail. The front remained relatively dry (with pore pressure dropping to zero) and of constant thickness, while the body elongated gradually in the course of the flow. Over the longest part of the flume, the basal pore pressure nearly matched the total normal stress, which means that shear strength was close to zero and the material was liquefied within the body [129]. Note that this behavior is consistent with the rheometrical data reported in Fig. 16, were data for material B did not show any yield stress in the short-term response to a shear-rate step.

Fig. 20 shows a sequence of aerial photographs taken when the material spread out on the runout surface. Self-organization of the slurry flow into a coarse-grained boundary and a muddy core became quite visible as the flow traveled the runout surface. Lateral levees were formed by the granular front and confined the ensuing muddy body. Note the levee formation is probably not induced by particle segregation since it is also observed for dry granular flows involving spherical equal-size particles [90].

In short, experiments performed by Parson et al. and Iverson et al. have shown that the flow of poorly sorted materials was characterized by the coexistence of two zones, each one with a distinctive rheological behavior: the flow border was rich in coarse-grained materials (Coulomb frictional behavior), while the core was fine-grained (viscoplastic behavior). This self-organization has a great influence on the flow behavior; notably the run-out distance can be significantly enhanced as a result of levee formation limiting lateral spreading. 


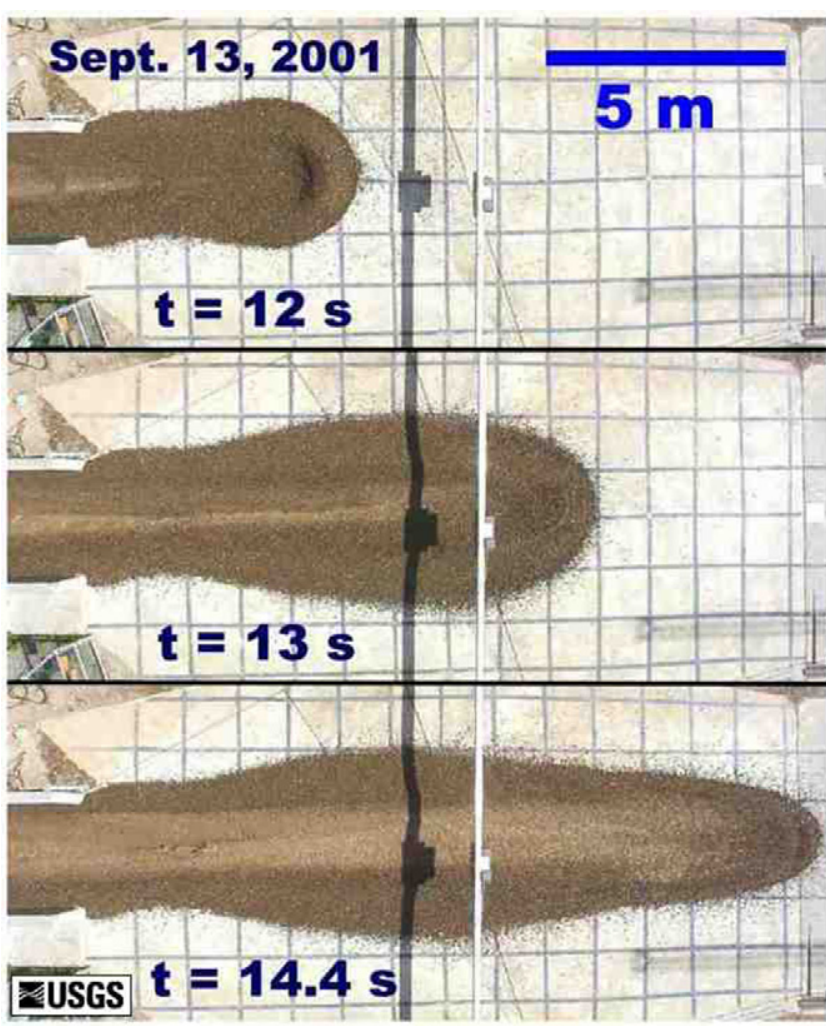

Fig. 20. Snapshots showing slurry flow discharging from the U.S. Geological Survey Debris-flow Flume and crossing the unconfined, nearly horizontal runout zone. The dark-toned material around the perimeter of the flow was predominantly gravel, while the light-toned material in the center of the flow was liquified mud. Figure reproduced from [127]; courtesy of Dr. R.M. Iverson.

\section{Application: sheet flows}

In this section, we will examine the consequences of the rheological properties the flow features have for thin freesurface flows (referred to as sheet flows), a typical flow configuration for geophysical flows. Different flow regimes can occur depending on the relative strength of inertial, pressure, and viscous contributions in the governing equations. In Section 4.1, dimensional analysis will be used to help clarify the notions of inertia-dominated and friction-dominated regimes. We will then focus on creeping flows on gentle slopes (Section 4.2) and fast flows (Section 4.3). In the analytical computations, we will use the shallowness of sheet flows to derive approximate equations. Since the Bingham model is the most studied and widespread constitutive equation, most examples will be based on this model, but we will also refer to papers dealing with alternative viscoplastic models or Coulomb friction.

\subsection{Scaling and flow regimes}

We consider a shallow layer of fluid flowing over a rigid impermeable plane inclined at an angle $\theta$ (see Fig. 21). The fluid is viscoplastic and incompressible; its density is denoted by $\rho$ and its bulk viscosity by $\eta=\tau / \dot{\gamma}$. The ratio $\epsilon=H_{*} / L_{*}$ between the typical vertical and horizontal lengthscales, $H_{*}$ and

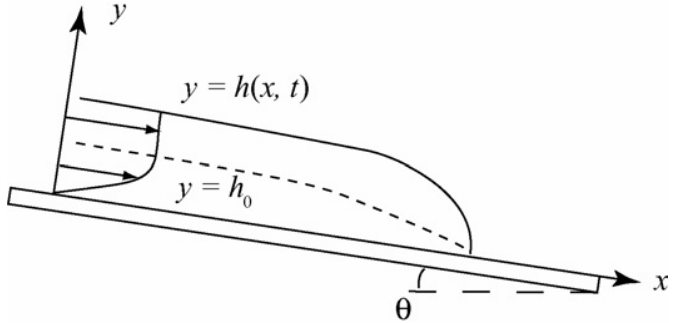

Fig. 21. The configuration of the flow.

$L_{*}$, respectively, is assumed to be small. The streamwise and vertical coordinates are denoted by $x$ and $y$, respectively.

A two-dimensional flow regime is assumed, namely any cross-stream variation is neglected. The depth of the layer is given by $h(x, t)$. The horizontal and vertical velocity components of the velocity $\mathbf{u}$ are denoted by $u$ and $v$, respectively. The fluid pressure is referred to as $p(x, y, t)$, where $t$ denotes time. The surrounding fluid (assumed to be air) is assumed to be dynamically passive (i.e., inviscid and low density compared to the moving fluid) and surface tension is neglected, which implies that the stress state at the free surface is zero.

The governing equations are given by the mass and momentum balance equations

$\nabla \cdot \mathbf{u}=0$

$\rho \frac{\mathrm{d} \mathbf{u}}{\mathrm{d} t}=\rho \frac{\partial \mathbf{u}}{\partial t}+\rho(\mathbf{u} \cdot \nabla) \mathbf{u}=\rho \mathbf{g}-\nabla p+\nabla \cdot \boldsymbol{\sigma}$,

supplemented by the following boundary conditions at the free surface

$v(x, h, t)=\frac{\mathrm{d} h}{\mathrm{~d} t}=\frac{\partial h}{\partial t}+u(x, h, t) \frac{\partial h}{\partial x}, v(x, 0, t)=0$.

There are many ways of transforming these governing equations into dimensionless expressions [20,140,149,191]. Here we depart slightly from the presentation given by Liu and Mei [149]. The characteristic streamwise and vertical velocities, the timescale, the typical pressure, and the order of magnitude of bulk viscosity are referred to as $U_{*}, V_{*}, T_{*}, P_{*}$, and $\eta_{*}$, respectively. Moreover, in addition to the lengthscale ratio $\epsilon$, we introduce the following dimensionless numbers that characterize free-surface, gravity-driven flows: the flow Reynolds number and the Froude number

$R e=\frac{\rho U_{*} H_{*}}{\eta_{*}} \quad$ and $\quad F r=\frac{U_{*}}{\sqrt{g H_{*} \cos \theta}}$.

The following dimensionless variables will be used in this section:

$\hat{u}=\frac{u}{U_{*}}, \quad \hat{v}=\frac{v}{V_{*}}, \quad \hat{x}=\frac{x}{L_{*}}, \quad \hat{y}=\frac{y}{H_{*}}, \quad$ and $\quad \hat{t}=\frac{t}{T_{*}}$

A natural choice for $T_{*}$ is $T_{*}=L_{*} / U_{*}$. The stresses are scaled as follows:

$\hat{\sigma}_{x x}=\frac{\eta_{*} U_{*}}{L_{*}} \sigma_{x x}, \hat{\sigma}_{x y}=\frac{\eta_{*} U_{*}}{H_{*}} \sigma_{x y}, \hat{\sigma}_{y y}=\frac{\eta_{*} U_{*}}{L_{*}} \sigma_{y y}$, and

$\hat{p}=\frac{p}{P_{*}}$ 
where $\sigma_{x x}, \sigma_{x y}$, and $\sigma_{y y}$ are the normal stress in the $x$ direction, the shear stress, and the normal stress in the $x$ direction, respectively. Here we are interested in free-surface flows. This leads us to set $P_{*}=\rho g H_{*} \cos \theta$, since we expect that, to leading order, the pressure adopts a hydrostatic distribution (see below). If we define the vertical velocity scale as $V_{*}=\epsilon U_{*}$, the mass balance Eq. (25) takes the following dimensionless form

$\frac{\partial \hat{u}}{\partial \hat{x}}+\frac{\partial \hat{v}}{\partial \hat{y}}=0$.

Substituting the dimensionless variables into the momentum balance Eq. (26) leads to

$\epsilon \operatorname{Re} \frac{\mathrm{d} \hat{u}}{\mathrm{~d} \hat{t}}=\frac{\epsilon \operatorname{Re}}{\operatorname{Fr}^{2}}\left(\frac{1}{\epsilon} \tan \theta-\frac{\partial \hat{p}}{\partial \hat{x}}\right)+\epsilon^{2} \frac{\partial \hat{\sigma}_{x x}}{\partial \hat{x}}+\frac{\partial \hat{\sigma}_{x y}}{\partial \hat{y}}$,
$\epsilon^{3} \operatorname{Re} \frac{\mathrm{d} \hat{v}}{\mathrm{~d} \hat{t}}=\frac{\epsilon \operatorname{Re}}{F^{2}}\left(-1-\frac{\partial \hat{p}}{\partial \hat{y}}\right)+\epsilon^{2} \frac{\partial \hat{\sigma}_{x y}}{\partial \hat{x}}+\epsilon^{2} \frac{\partial \hat{\sigma}_{y y}}{\partial \hat{y}}$.

The momentum balance equation expresses a balance between gravity acceleration, inertial terms, pressure gradient, and viscous dissipation, whose order of magnitude is $\rho g \sin \theta, \rho U_{*}^{2} / L_{*}$, $P_{*} / L_{*}$, and $\eta_{*} U_{*} / H_{*}^{2}$, respectively. Depending on the values considered for the characteristic scales, different types of flow regime occur. At least four regimes, where two contributions prevail compared to the others, could be achieved in principle

(1) Inertial regime, where inertial and pressure-gradient terms are of the same magnitude. We obtain

$U_{*}=\sqrt{g H_{*} \cos \theta}$.

The order of magnitude of the shear stress is $\partial \sigma_{x y} / \partial y=$ $\rho g O\left(\epsilon^{-1} R e^{-1}\right)$. This regime occurs when: $\epsilon \operatorname{Re} \gg 1$ and $\mathrm{Fr}=O(1)$.

(2) Viscous regime, where the pressure gradient is balanced by viscous stresses within the bulk. In that case, we have

$U_{*}=\frac{\rho g \cos \theta H_{*}^{3}}{\eta_{*} L_{*}}$.

Inertial terms must be low compared to the pressured gradient and the slope must be gentle $(\tan \theta \ll \epsilon)$. This imposes the following constraint: $\epsilon R e \ll 1$. We deduced that $F r^{2}=O(\epsilon R e) \ll 1$.

(3) Visco-inertial regime, where inertial and viscous contributions are nearly equal. In that case, we have

$U_{*}=\frac{1}{\epsilon} \frac{\eta_{*}}{\rho H_{*}}$.

The pressure gradient must be low compared to the viscous stress, which entails the following condition $\eta_{*} \gg$ $\epsilon \rho \sqrt{g H_{*}^{3}}$. We obtain $\epsilon \operatorname{Re} \sim 1$ and $F r=\eta_{*} /\left(\rho \epsilon \sqrt{g H_{*}^{3}}\right) \gg$ 1.

(4) Nearly steady uniform regime, where the viscous contribution matches gravity acceleration. In that case, we have

$U_{*}=\frac{\rho g \sin \theta H_{*}^{2}}{\eta_{*}}$.

Inertia must be negligible, which means $\epsilon \ll 1$ (stretched flows). We obtain $\operatorname{Re}=O\left(F r^{2}\right)$ and $\tan \theta \gg \epsilon$ (mild slopes).
In the inertial regime, the rheological effects are so low that they can be neglected and the final governing equations are the Euler equations. The visco-inertial regime is more spurious and has no specific interest in geophysics, notably because the flows are rapidly unstable. More interesting is the viscous regime that may achieved for very slow flows on gentle slopes $(\theta \ll 1)$, typically when flows come to rest. We will further describe this regime in Section 4.2. When there is no balance between two contributions, we have to solve the full governing equations. This is usually a difficult task, even numerically. To simplify the problem, one can use flow-depth averaged equations (see Section 4.3). The nearly-steady regime will be exemplified in Section 4.3 within the framework of the kinematic-wave approximation. Finally, it should be kept in mind that the partitioning into four regimes holds for viscous (Newtonian) fluids and non-Newtonian materials for which the bulk viscosity does not vary significantly with shear rate over a sufficiently wide range of shear rates. In the converse case, further dimensionless groups (e.g., the Bingham number $\left.\mathrm{Bi}=\tau_{\mathrm{c}} H_{*} /\left(\mu U_{*}\right)\right)$ must be introduced, which makes this classification more complicated.

\subsection{Slow motion}

Slow motion of a viscoplastic material has been investigated by Liu and Mei [149,150], Mei [171], Mei and Yuhi [170], Coussot et al. [68,69], Balmforth and Craster [20,23], and Matson and Hogg [165]. Taking the two dominant contributions in Eqs. (29) and (30) and returning to the physical variables, we deduce

$\sigma_{x y}=\rho g \cos \theta(h-y)\left(\tan \theta-\frac{\partial h}{\partial x}\right)$

$p=\rho g(h-y) \cos \theta$.

The bottom shear stress is then found to be $\tau_{\mathrm{b}}=\left.\sigma_{x y}\right|_{y=0}$. For bottom shear stresses in excess of the yield stress $\tau_{\mathrm{c}}$, flow is possible. When this condition is satisfied, there is a yield surface at depth $y=h_{0}$ within the bulk, along which the shear stress matches the yield stress

$\left.\sigma_{x y}\right|_{y=h_{0}}=\rho g \cos \theta\left(h-h_{0}\right)\left(\tan \theta-\frac{\partial h}{\partial x}\right)=\tau_{\mathrm{c}}$.

The yield surface separates the flow into two layers [20,149]: the bottom layer, which is sheared, and the upper layer or plug layer, where the shear rate is nearly zero. Indeed, using an asymptotic analysis, Balmforth and Craster demonstrated that in the socalled plug layer, the shear rate is close to zero, but nonzero [20]. This result may be seen as anecdotic, but it is in fact of great importance since it resolves a number of paradoxes raised about viscoplastic solutions [2,148].

On integrating the shear-stress distribution, we can derive a governing equation for the flow depth $h(x, t)$. For this purpose, we must specify the constitutive equation. For the sake of simplicity, we consider a Bingham fluid in one-dimensional flows as Liu and Mei [149] did; the extension to Herschel-Bulkley and/or two-dimensional flows can be found in $[20,23,170]$. In 
the sheared zone, the velocity profile is parabolic

$u(y)=\frac{\rho g \cos \theta}{\mu}\left(\tan \theta-\frac{\partial h}{\partial x}\right)\left(h_{0} y-\frac{1}{2} y^{2}\right) \quad$ for $y \leq h_{0}$,

while the velocity is constant to leading order within the plug

$u(y)=u_{0}=\frac{\rho g h_{0}^{2} \cos \theta}{\mu}\left(\tan \theta-\frac{\partial h}{\partial x}\right)$ for $y \geq h_{0}$,

The flow rate is then

$q=\int_{0}^{h} u(y) \mathrm{d} y=\frac{\rho g h_{0}^{2}\left(3 h-h_{0}\right) \cos \theta}{6 \mu}\left(\tan \theta-\frac{\partial h}{\partial x}\right)$.

Integrating the mass balance equation over the flow depth provides

$\frac{\partial h}{\partial t}+\frac{\partial q}{\partial x}=0$.

Substituting $q$ with its expression (34) and the yield surface elevation $h_{0}$ with Eq. (33) into Eq. (35), we obtain a governing equation for $h$, which takes the form of a nonlinear diffusion equation

$\frac{\partial h}{\partial t}=\frac{\partial}{\partial x}\left[F\left(h, h_{0}\right)\left(\frac{\partial h}{\partial x}-\tan \theta\right)\right]$,

with $F=\rho g h_{0}^{2}\left(3 h-h_{0}\right) \cos \theta /(6 \mu)$.

A typical application of this analysis is the derivation of the shape of a viscoplastic deposit. Contrary to a Newtonian fluid, the flow depth of a viscoplastic fluid cannot decrease indefinitely when the fluid spreads out along an infinite plane. Because of the finite yield stress, when it comes to rest, the fluid exhibits a nonuniform flow-depth profile, where the pressure gradient is exactly balanced by the yield stress. On an infinite horizontal plane, the bottom shear stress must equal the yield stress. Using Eq. (31) with $\theta=0$ and $y=0$, we eventually obtain [149]

$\left.\sigma_{x y}\right|_{y=0}=\tau_{\mathrm{c}}=-\rho g h \frac{\partial h}{\partial x}$,

which, on integrating, provides

$h(x)-h_{i}=\sqrt{\frac{2 \tau_{\mathrm{c}}}{\rho g}\left(x_{i}-x\right)}$,

where $h=h_{i}$ at $x=x_{i}$ is a boundary condition. This equation shows that the deposit-thickness profile depends on the square root of the distance. When the slope is nonzero, an implicit solution for $h(x)$ to Eq. (31) is found [149]

$$
\begin{aligned}
& \tan \theta\left(h(x)-h_{i}\right)+\frac{\tau_{\mathrm{c}}}{\rho g \cos \theta} \log \left[\frac{\tau_{\mathrm{c}}-\rho g h \sin \theta}{\tau_{\mathrm{c}}-\rho g h_{i} \sin \theta}\right] \\
& =\tan ^{2} \theta\left(x-x_{i}\right) .
\end{aligned}
$$

The shape of a static two-dimensional pile of viscoplastic fluid was investigated by Coussot et al. [69], Mei and Yuhi [170], Osmond and Griffiths [185], and Balmforth et al. [23]; the latter derived an exact solution, while the former authors used numerical methods or ad hoc approximations to solve the two-dimensional equivalent to Eq. (31). Similarity solutions to Eq. (36) have also been provided by Balmforth et al. [23] in the case of a viscoplastic flow down a gently inclined, unconfined surface with a time-varying source at the inlet.

\subsection{Fast motion}

The most common method for solving fast-motion freesurface problems is to depth-average the local equations of motion. In the literature, this method is referred to as the SaintVenant approach, the boundary-layer approximation, the lubrication approximation, the long-wave approximation, etc. Here, by fast motion, we refer to situations where inertia, rheological effects, and pressure play all a role in flow dynamics. However, the flow velocity must not be too high; otherwise instabilities occur at the free surface $[24,65,151,229]$.

The Saint-Venant approach involves integrating the momentum and mass balance equations over the depth. A considerable body of work has been published on this method for Newtonian and non-Newtonian fluids, including viscoplastic $[64,65,116,117,191,223]$ and granular materials $[45,61,101,102,130,142,161,195,199,212]$. Here, we shall briefly recall the principle and then directly provide the resulting governing equations. Let us start with the local mass balance (25). Integrating this equation over the flow depth leads to

$$
\begin{aligned}
& \int_{0}^{h(x, t)}\left(\frac{\partial u}{\partial x}+\frac{\partial v}{\partial y}\right) \mathrm{d} y \\
& \quad=\frac{\partial}{\partial x} \int_{0}^{h} u(x, y, t) \mathrm{d} y-u(h) \frac{\partial h}{\partial x}-v(x, h, t)-v(x, 0, t) .
\end{aligned}
$$

At the free surface and the bottom, the $y$-component of velocity $v$ satisfies the boundary conditions (27). We then easily deduce

$\frac{\partial h}{\partial t}+\frac{\partial h \bar{u}}{\partial x}=0$

where we have introduced depth-averaged variables defined as

$\bar{f}(x, t)=\frac{1}{h(x, t)} \int_{0}^{h(x, t)} f(x, y, t) \mathrm{d} y$.

The same procedure is applied to the momentum balance Eq. (26). Without any difficulty, we can deduce the averaged momentum equation from the $x$-component of the momentum equation

$\bar{\rho}\left(\frac{\partial h \bar{u}}{\partial t}+\frac{\partial h \overline{u^{2}}}{\partial x}\right)=\bar{\rho} g h \sin \theta-\frac{\partial h \bar{p}}{\partial x}+\frac{\partial h \bar{\sigma}_{x x}}{\partial x}-\tau_{\mathrm{b}}$,

where we have introduced the bottom shear stress: $\tau_{\mathrm{b}}=$ $\sigma_{x y}(x, 0, t)$. In the present form, the system of Eqs. (40) and (41) is not closed since the number of variables exceeds the number of equations. A common approximation involves introducing a parameter (sometimes called the Boussinesq momentum coefficient), which links the mean velocity to the mean square velocity

$\overline{u^{2}}=\frac{1}{h} \int_{0}^{h} u^{2}(y) \mathrm{d} y=\alpha \bar{u}^{2}$.

Most of the time, the coefficient $\alpha$ is set to unity.

Another helpful (and common) approximation, not mentioned in the above system, concerns the computation of stress. Within the framework of long wave approximation, we assume that longitudinal motion outweighs vertical motion: for any quantity $m$ related to motion, we have $\partial m / \partial y \gg \partial m / \partial x$. This 
allows us to consider that every vertical slice of flow can be treated as if it was locally uniform. In such conditions, it is possible to infer the bottom shear stress by extrapolating its steadystate value and expressing it as a function of $u$ and $h$. Using this approximation, Coussot $[64,65]$ obtained the following bottom shear stress

$\tau_{\mathrm{b}}=\mu\left(\frac{1+2 n}{1+n}\right)^{n} \frac{\bar{u}^{n}}{h_{0}^{n+1}\left(\left(2+n^{-1}\right) h-h_{0}\right)^{n}}$,

for Herschel-Bulkley fluids. Using the first-order approximation of the $y$-component of the momentum balance Eq. (26), he found that the pressure was hydrostatic, which leads to a flow-depth averaged pressure

$\bar{p}=\frac{1}{2} \rho g h \cos \theta$.

The effects of normal stresses can be neglected to first order. Note that this derivation is not the only way of deriving the SaintVenant equations for a Bingham fluid; alternative procedures have been proposed [116,117,189]. For instance, Huang and Garcia further considered two partial differential equations to supplement the governing Eqs. (40) and (41) [116,117]: one equation governing the elevation $h_{0}$ of the yield surface and another providing the bottom shear stress.

For Coulomb materials, the same procedure can be repeated. The only modification concerns the momentum balance Eq. (41), which takes the form [130,212]

$\bar{\rho}\left(\frac{\partial h \bar{u}}{\partial t}+\frac{\partial h \bar{u}^{2}}{\partial x}\right)=\bar{\rho} g h\left(\sin \theta-k \cos \theta \frac{\partial h}{\partial x}\right)-\tau_{\mathrm{b}}$,

with $k$ a proportionality coefficient between the normal stresses $\bar{\sigma}_{x x}$ and $\bar{\sigma}_{y y}$, which is computed by assuming limiting Coulomb equilibrium in compression $\left(\partial_{x} \bar{u}<0\right)$ or extension $\left(\partial_{x} \bar{u}>0\right)$; the coefficient is called the active/passive pressure coefficient. In Eq. (43), the bottom shear stress can be computed by using the Coulomb law $\tau_{\mathrm{b}}=\left(\left.\bar{\sigma}_{y y}\right|_{y=0}-p_{\mathrm{b}}\right) \tan \varphi$, with $\left.\bar{\sigma}_{y y}\right|_{y=0}=$ $\bar{\rho} g h \cos \theta$ and $p_{\mathrm{b}}$ the pore pressure at the bed level.

Analytical solutions can be obtained for the Saint-Venant equations. Most of them were derived by seeking self-similarity solutions (see [61,212,214] for the Coulomb model and [114] for viscoplastic and hydraulic models). Some solutions can also be obtained using the method of characteristics. We are going to see two applications based on these methods.

In the first application, we use the fact that the Saint-Venant equations for Coulomb materials are structurally similar to those used in hydraulics when the bottom drag can be neglected. The only difference lies in the nonhydrostatic pressure term and the source term (bottom shear stress). However, using a change in variable makes it possible to retrieve the usual shallow-water equations and seek similarity solutions to derive the Ritter solutions $[84,139,142,161,220]$. The Ritter solutions are the solutions to the so-called dam-break problem, where an infinite volume of material at rest is suddenly released and spreads over a dry bed (i.e., no material laying along the bed). Much attention has been paid to this problem, notably in geophysics because it is used as a paradigm for studying rapid surge motion. We pose

$x^{*}=x-\frac{1}{2} \delta t^{2}, t^{*}=t, u^{*}=u-\delta t, \quad$ and $\quad h^{*}=h$, where we introduced the parameter $\delta=g \cos \theta(\tan \theta-\mu)$. We deduce

$$
\begin{aligned}
& \frac{\partial h^{*}}{\partial t^{*}}+\frac{\partial h^{*} u^{*}}{\partial x^{*}}=0, \\
& \frac{\partial u^{*}}{\partial t^{*}}+u^{*} \frac{\partial u^{*}}{\partial x^{*}}+g k \cos \theta \frac{\partial h^{*}}{\partial x^{*}}=0 .
\end{aligned}
$$

For the dam-break problem, the initial and boundary conditions are

$$
\begin{gathered}
-\infty<x<\infty, u(x, 0)=0, \\
x<0, h(x, 0)=h_{i}, \\
x>0, h(x, 0)=0 .
\end{gathered}
$$

The analytical solutions to Eqs. (44) and (45) are the well-known Ritter solutions. We are looking for a similarity solution in the form [100]

$\bar{u}^{*}=t^{* \beta / \alpha} U\left(\zeta^{*}\right)$ and $h^{*}=t^{* \gamma / \alpha} H\left(\zeta^{*}\right)$,

with $\zeta^{*}=x^{*} / t^{* \alpha}$ the similarity variable, and $H$ and $U$ two unknown functions. Substituting $\bar{u}^{*}$ and $h^{*}$ with their similarity forms into (44) and (45), we find: $\beta+\alpha=1$ and $\gamma+2 \alpha=2$. For this solution to satisfy the initial and boundary conditions, we must pose $\beta=\gamma=0$, hence $\alpha=1$. We then infer

$$
\left(\begin{array}{cc}
H & U-\zeta^{*} \\
U-\zeta^{*} & k g \cos \theta
\end{array}\right) \cdot\left(\begin{array}{c}
U^{\prime} \\
H^{\prime}
\end{array}\right)=0
$$

where the prime denotes the $\zeta^{*}$-derivative. For this system to admit a nonconstant solution, its determinant must vanish, which leads to $\mathrm{kg} H \cos \theta=\left(U-\zeta^{*}\right)^{2}$. On substituting this relation into the system above, we deduce $U^{\prime}=2 \zeta^{*} / 3$, thus $U=2\left(\zeta^{*}+c\right) / 3$, where $c$ is a constant of integration, $H=4\left(c-(1 / 2) \zeta^{*}\right)^{2} /(9 \mathrm{~kg} \cos \theta)$. The constant $c$ is found using the boundary conditions and by assuming that the undisturbed flow slides at constant velocity $\delta t: c=\sqrt{\mathrm{kg} h_{i} \cos \theta}$. Returning to the original variables, we find

$\bar{u}(x, t)=\bar{u}^{*}+\delta t=\frac{2}{3}\left(\frac{x}{t}+\delta t+c\right)$,

$h(x, t)=\frac{1}{9 \mathrm{~kg} \cos \theta}\left(-\frac{x}{t}+\frac{\delta}{2} t+2 c\right)^{2}$.

The boundary conditions also imply that the solution is valid over the $\zeta$-range $[-c-\delta t, 2 c+\delta t / 2]$; the lower bound corresponds to the upstream condition $\bar{u}=0$, while the upper bound is given by the downstream condition $h=0$. It is worth noting that the front velocity $u_{\mathrm{f}}=2 c+\delta t / 2$ is constantly increasing or decreasing depending on the sign of $\delta$. When $\delta<0$ (friction in excess of slope angle), the front velocity vanishes at $t=4 c /|\delta|$. Fig. 22 shows that the shape of the tip region is parabolic at short times $(\delta t \ll c)$, in agreement with experimental data [21,221]. Solutions corresponding to finite released volumes were also obtained by Dressler [84] and Savage [212,214].

In the second application, we use the method of characteristics to find a solution to the governing equations for Bingham flows that are stretched thin when they are nearly steady uniform. In Section 4.1, we found that for mild slopes, when the aspect ratio $\epsilon$ is very low, the inertial and pressure contributions can 


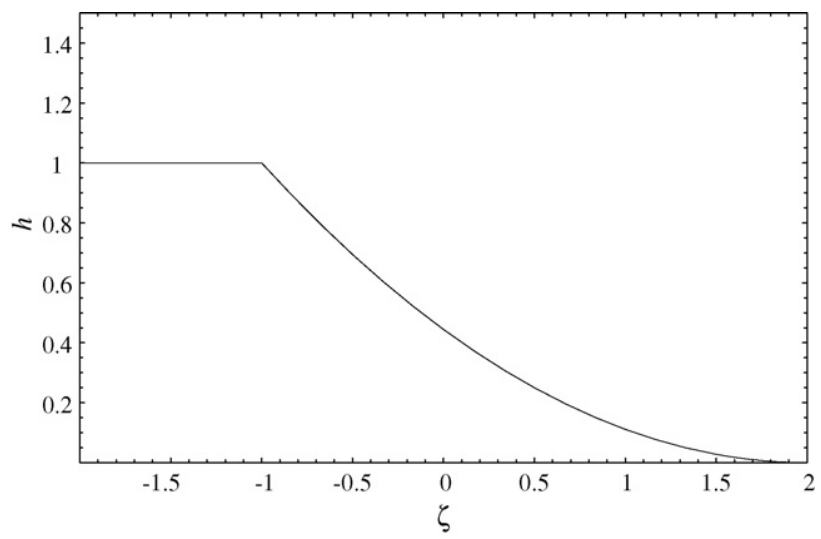

Fig. 22. Flow-depth profile generated just after the wall retaining a granular material is removed. Computations made with $c=1 \mathrm{~m} / \mathrm{s}$. The similarity variable $\zeta$ is $\zeta=x / t$.

be neglected. This means that the flow-depth averaged velocity is very close to the mean velocity reached for steady uniform flows

$\bar{u}_{\mathrm{s}}=u_{\mathrm{p}}\left(1-\frac{h_{0}}{3 h}\right)$,

where $u_{\mathrm{p}}$ is the plug velocity

$u_{\mathrm{p}}=\frac{\rho g h_{0}^{2} \sin \theta}{2 \mu}$,

with $h$ the flow depth and $h_{0}=h-\tau_{\mathrm{c}} /(\rho g \sin \theta)$ the yieldsurface elevation; $h_{0}$ must be positive or no steady flow occurs. We then use the kinematic-wave approximation introduced by Lighthill and Whitham [147] to study floods on long rivers; this approximation was then extensively used in hydraulic applications $[16,116,117,119,236]$. It involves substituting the mean velocity into the mass balance Eq. (40) by its steady-state value

$\frac{\partial h}{\partial t}+\frac{\partial}{\partial x} u_{\mathrm{p}}\left(h-\frac{h_{0}}{3}\right)=0$.

Introducing the plug thickness $h_{\mathrm{p}}=h-h_{0}=\tau_{\mathrm{c}} /(\rho g \sin \theta)$, we obtain an expression that is a function of $h$ and its time and space derivative

$\frac{\partial h}{\partial t}+K\left(h^{2}-h h_{\mathrm{p}}\right) \frac{\partial h}{\partial x}=0$,

with $K=\rho g \sin \theta / \mu$. The governing equation takes the form of a nonlinear advection equation, which can be solved using the method of characteristics [145].

Using the chain rule for interpreting this partial differential Eq. (49), we can show that it is equivalent to the following ordinary equation

$\frac{\mathrm{d} h}{\mathrm{~d} t}=0$,

along the characteristic curve

$\frac{\mathrm{d} x}{\mathrm{~d} t}=\lambda(h)$,

in the $(x, t)$ plane, with $\lambda(h)=K h\left(h-h_{\mathrm{p}}\right)$. Eq. (50) shows that the flow depth is constant along the characteristic curve, hence the characteristic curves are straight lines, the slope of which are given by the right-hand side term $\lambda(h)$ in Eq. (51). These characteristic curves can be used to solve an initial value problem, where the initial value of $h$ is known over a given interval: $h=h_{i}\left(x_{i}\right)$ (at $\left.t=0\right)$. The value of $h$ along each characteristic curve is the value of $h$ at the initial point $x(0)=x_{i}$. We can thus write

$h(x, t)=h_{i}\left(x_{i}\right)=h_{i}\left(x-\lambda\left(h_{i}\left(x_{i}\right)\right) t\right)$.

It is worth noting that because of the nonlinearity of Eq. (49), a smooth initial condition can generate a discontinuous solution (shock) if the characteristic curves intersect, since at the point of intersection $h$ takes (at least) two values [145]. An interesting related issue is the Riemann problem, where we seek a solution to the nonlinear advection Eq. (49) when the initial condition is discontinuous and step-shaped (see Eq. (46) for the initial conditions). Here, this problem is of particular interest not only for developing numerical algorithms, but also for finding solutions to the dam-break problem. It can be shown that, when the bed is dry ahead of the front, the solution takes the form of a simple wave or rarefaction wave, i.e., a continuous similarity solution to Eq. (49), which links the material still at rest behind and the surge tip. Indeed, if we seek similarity solutions to Eq. (49) in the form $h=t^{\alpha} H(\zeta)$, with $|\zeta|=x / t^{\beta}$, we find on substituting this form into Eq. (49) that $\alpha=0$ and $\beta=1$; here we pose $h=H(-x / t)$. Furthermore, $H$ is solution of the equation $H^{\prime}\left(\zeta-K H\left(H-h_{\mathrm{p}}\right)\right)=0$,

from which we deduce that either $H$ is constant or satisfies the quadratic equation $\zeta-K H\left(H-h_{\mathrm{p}}\right)=0$. Solving this equation we find

$h=\frac{h_{\mathrm{p}}}{2}\left(1+\sqrt{1-\frac{4}{K h_{\mathrm{p}}^{2}} \frac{x}{t}}\right)$,

defined for $x \leq \dot{x}_{\mathrm{f}} t$ with $\dot{x}_{\mathrm{f}}=K h_{\mathrm{p}}^{2} / 4$. Contrary to the Ritter solution for water, the flow-depth profile presents a steep nose at the front (confusingly called shock in earlier work) and is concave backward, as shown in the numerical example of Fig. 23. Note that the flow depth at the front is exactly half the plug thickness $h_{\mathrm{p}}$. The front moves at constant velocity $\dot{x}_{\mathrm{f}}$. Note that the solution given here differs from the approximate solution provided in the engineering literature $[16,116,119]$, where a constraint

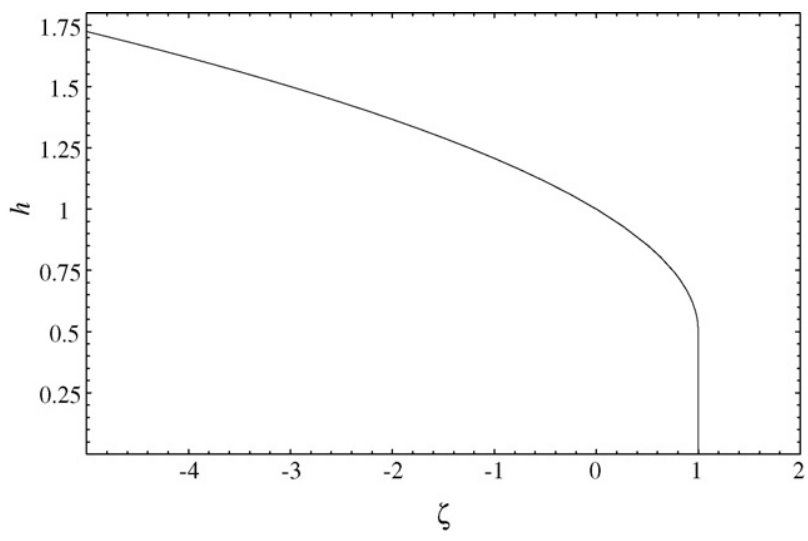

Fig. 23. Flow-depth profile of the viscoplastic simple wave generated after the wall is removed. Computations made with $K=4 \mathrm{~m}^{-1} \mathrm{~s}^{-1}$ and $h_{\mathrm{p}}=1 \mathrm{~m}$. 
on the volume released was used to compute the front position. It is also worth wondering whether the approximation of the kinematic wave can be used to provide a correct solution to the dam-break problem, where both inertia and pressure gradient should be taken into account.

\section{Field evidence}

\subsection{Using historical or monitored events}

For a long time, the only source of information was the traces of past events [132]. For instance, measuring the flow-depth profile of a debris flow deposit and using the flow-depth profile Eq. (38) for a Bingham fluid makes it possible to derive the yield stress $[69,207]$; the mean flow thickness of a muddy debris flow in straight channels or the slope angle of a coarse-grained deposit can also be used to infer the yield stress $\tau_{\mathrm{c}}$ or the friction angle $\varphi$. Figs. 24 and 25 shows typical examples of debris-flow deposit. In Fig. 24, the deposit is a lateral levee left by a granular debris flow, which is characterized by a nearly straight free surface. In Fig. 25, the deposit profile is nearly parabolic, which is interpreted as the hallmark of viscoplastic behavior (see Eq. 38). Another example is provided by superelevation in channel bends. Indeed in the course of an avalanche or a debris flow, the flowing material sometimes encounters curved channel bends, which cause the material to superelevate or climb up on the bend side because of centrifugal forces. The level of flowing material is higher on the outward side than on the inward side. This can provide information on mean velocity at that location [228, 133, 168].

Over the last two decades, an increasing number of sites throughout the world have been equipped with sensors and videorecorders, such as the Illgraben torrent (debris flow) [166,167], the Schipfenbach stream [121], and the Vallée de la Sionne (snow avalanches) in Switzerland [4], the Acquabona river in Italy [42], the Col du Lautaret (snow avalanches) [172] in France, etc. Monitored and historical events have been used to backcalculate the constitutive parameters by matching the field data (run-out distance, flow-rate, etc.) and the model's predictions $[25,50,77,81,237,242]$. This, however, does not provide evi-

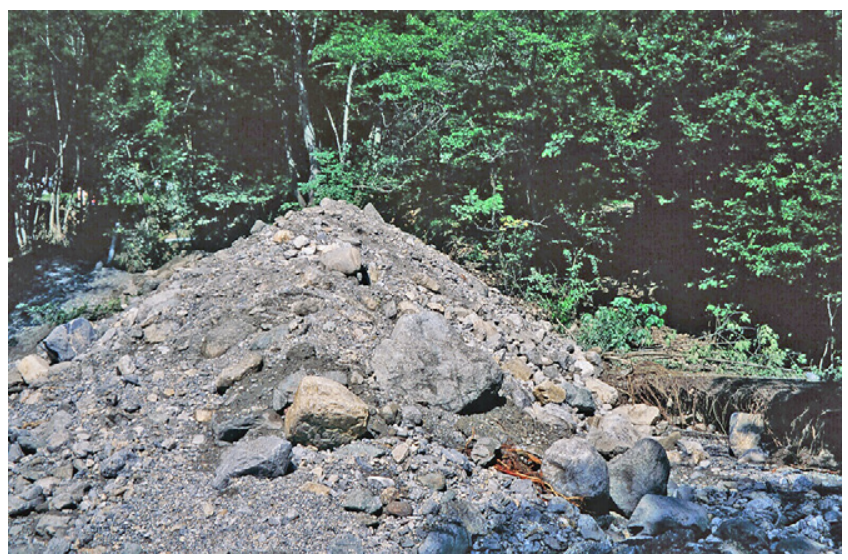

Fig. 24. Lateral levee of a coarse-grained debris flow in the Bez torrent (France, 24 July 1995). The levee looks like a unconsolidated, noncohesive granular pile.

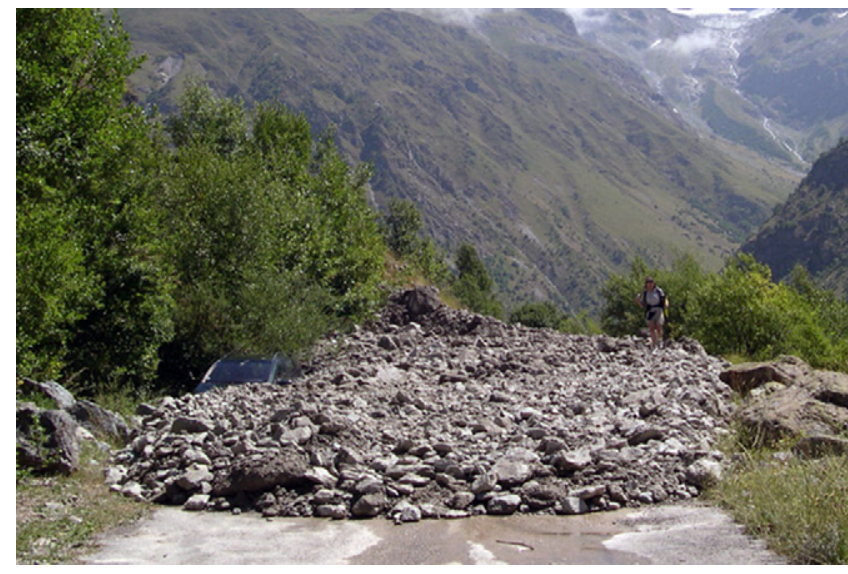

Fig. 25. Deposit lobe of a poorly sorted debris flow in the Valgaudemar valley (France, 30 July 2003), caused by heavy rainfalls. The deposit-thickness profile exhibits a parabolic shape.

dence that the constitutive equation is appropriate. Occasionally, some useful information such as the velocity profile within the bulk has been obtained; for instance, Gubler [104] took measurements on real avalanches using a Doppler radar. He found that the velocity profile inside the observed avalanches exhibited a plug flow (constant-velocity zone) and a sheared zone at the bottom, clearly revealing that there was shear localization at the bottom.

\subsection{Inferring rheological information from velocity records}

If we wish to derive rheological information from field data, the first idea would be to extend viscometric methods (e.g., the method for deriving the flow curve from the flow-velocity/flowdepth relationship [13]) or to develop inverse-problem techniques (e.g., see [209]), where information can be inferred from field data by assuming a particular form of the governing equations [e.g., the sheet-flow Eqs. (40)-(41)]. In practice, however, this idea is of limited interest given how difficult it is to obtain field measurements of both the flow depth and mean velocity. In most cases, the only information available is the front velocity, which substantially the possibility of inferring rheological information.

However, the idea deserves further development by simplifying the equations of motion. Here, the simplest case where the fluid can be considered a slender sliding body, of volume $V$ and mass $m$, is examined. Investigating this simplified case, Ancey and Meunier [12] performed a back analysis on 15 welldocumented avalanches by inferring the bulk frictional force from avalanche velocity. In their treatment, the avalanche is assumed to behave as a rigid body, which moves along a curvilinear two-dimensional profile, whose equation in a Cartesian frame takes the form: $y=z(x)$, where $y$ is the elevation and $x$ is an arbitrary distance measured along a horizontal axis (see Fig. 26). The curvature radius is denoted by $R$. The sliding body experiences a frictional force, the tangential and normal components of which are denoted by $F_{\mathrm{t}}$ and $F_{\mathrm{n}}$, respectively.

The position of the center of mass is given by its curvilinear abscissa $\xi=\int_{0}^{x} \sqrt{1+z_{x}^{2}\left(x^{\prime}\right)} \mathrm{d} x^{\prime}$ (where $z_{x}$ is the $x$-derivative of $z$ ). Therefore, we have $x=\xi \cos \bar{\theta}$, with $\bar{\theta}$ the mean path inclina- 


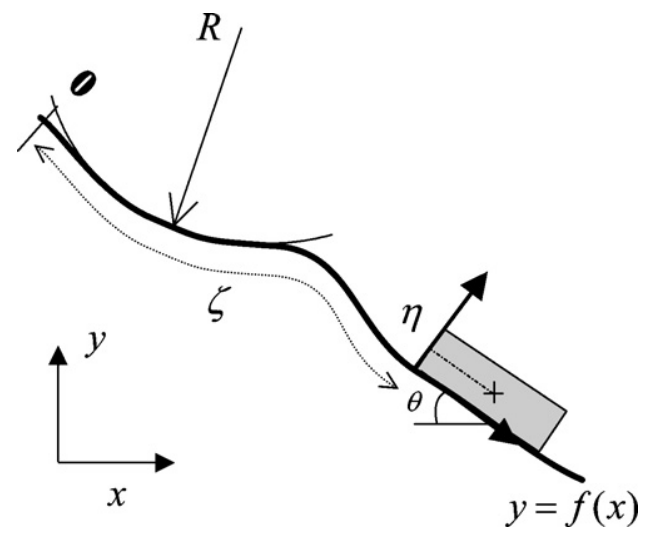

Fig. 26. Curvilinear frame related to the one-dimensional path profile traveled by avalanches.

tion computed over the interval $[0, x]$. The ordinate of the center of mass (relative to the curve $z$ ) is denoted by $\eta$. In the natural basis $\left(\mathbf{e}_{1}, \mathbf{e}_{2}\right)$ associated with the curvilinear coordinates $(\xi, \eta)$, the contravariant components of the velocity vector are denoted by $\left(u^{(1)}, u^{(2)}\right)=(\mathrm{d} \xi / \mathrm{d} t, \mathrm{~d} \eta / \mathrm{d} t)$ and its physical components are given by $\left(u^{\langle 1\rangle}, u^{\langle 2\rangle}\right)=\left((1-\eta / R) u^{(1)}, u^{(2)}\right)$. The contravariant components of acceleration in the natural basis are

$a^{(1)}=\frac{\mathrm{d}^{2} \xi}{\mathrm{d} t^{2}}+\Gamma_{11}^{1}\left(\frac{\mathrm{d} \xi}{\mathrm{d} t}\right)^{2}+2 \Gamma_{12}^{1} \frac{\mathrm{d} \xi}{\mathrm{d} t} \frac{\mathrm{d} \eta}{\mathrm{d} t}+\Gamma_{22}^{1}\left(\frac{\mathrm{d} \eta}{\mathrm{d} t}\right)^{2}$,

$a^{(2)}=\frac{\mathrm{d}^{2} \eta}{\mathrm{d} t^{2}}+\Gamma_{11}^{2}\left(\frac{\mathrm{d} \xi}{\mathrm{d} t}\right)^{2}+2 \Gamma_{12}^{2} \frac{\mathrm{d} \xi}{\mathrm{d} t} \frac{\mathrm{d} \eta}{\mathrm{d} t}+\Gamma_{22}^{2}\left(\frac{\mathrm{d} \eta}{\mathrm{d} t}\right)^{2}$,

where $\Gamma_{i j}^{k}$ are the Christoffel symbols. Because the natural basis is orthogonal, the Christoffel coefficients are zero, except for $\Gamma_{12}^{1}=\Gamma_{21}^{1}=-C /(1-C \eta), \Gamma_{11}^{2}=C(1-C \eta)$, and $\Gamma_{11}^{1}=$ $-\eta(\mathrm{d} C / \mathrm{d} \xi) /(1-C \eta)$, where $C=1 / R$ is the curvature. The velocity in the $\xi$-direction is $u=u^{\langle 1\rangle}=(1-\eta C) \mathrm{d} \xi / \mathrm{d} t ; \eta$ is fairly constant and the velocity $u^{\langle 2\rangle}$ in the $\eta$-direction is close to zero. The downward and normal components of the momentum equation can be expressed in the physical curvilinear basis as

$$
\begin{aligned}
& \left(1-\frac{\eta}{R}\right)^{2} \frac{\mathrm{d}^{2} \xi}{\mathrm{d} t^{2}}+\frac{\eta}{R^{2}} \frac{\mathrm{d} R}{\mathrm{~d} \xi}\left(\frac{\mathrm{d} \xi}{\mathrm{d} t}\right)^{2}=g \sin \theta(\xi)-\frac{F_{\mathrm{t}}}{m}, \\
& -\frac{1}{R-\eta}\left(\frac{\mathrm{d} \xi}{\mathrm{d} t}\right)^{2}=-g \cos \theta(\xi)+\frac{F_{\mathrm{n}}}{m} .
\end{aligned}
$$

On the left-hand side of (52), the first term represents the downward component of the acceleration, while the second term reflects the radial effect due to the curvature of the path profile. On the right-hand side of (52), the first contribution is the driving action of gravity while the second term stands for the frictional force exerted by the bottom (ground or snowcover) on the avalanche.

The interpretation of Eqs. (52) and (53) is clear: if one has a record yielding the body velocity as a function of the position along the path, then it is possible to directly deduce the frictional force components and its relationship with the velocity $u$ to a multiplicative factor $m$. To first order ( $R$ being very large in most cases), the average normal force only depends on the local slope: $F_{\mathrm{n}}=m g \cos \theta(\xi)$. Eq. (52) should provide the

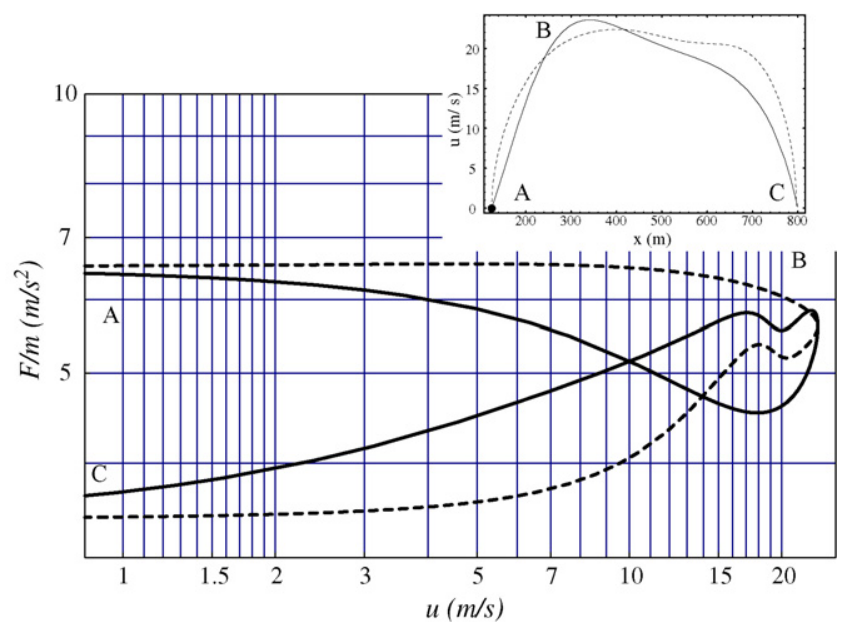

Fig. 27. Variation in the frictional force per unit mass $F / m$ with the avalanche velocity $u$ for the avalanche of 21 December 1997 at the Arabba site (solid line); $F / m$ was obtained by applying Eq. (52) to the measured velocities and path profile, both regularized using Legendre polynomials. The dashed curve stands for the variation in the driving force per unit mass $g \sin \theta$. In the inset, we have reported the variations in the measured velocities (dots) with downstream distance $x$. In the inset, the solid line represents the interpolated velocities while the dashed line stands for the velocity of a rigid body sliding in a purely Coulombic regime (with $f=0.66$ ). Letters from A to $\mathrm{C}$ refer to various stages of the avalanche run (see text). After [12].

main trends of the rheological behavior. Plotting the resulting force per unit mass in a phase space $(u, F / m)$ can give an idea of the dependence of the frictional force on the mean velocity and normal component.

For most events, the frictional force was found to be weakly dependent on velocity or to fluctuate around a mean value during the entire course of the avalanche. Fig. 27 shows a typical example provided by the avalanche at the Arraba site (Italy) on 21 December 1997. This figure reports the variation in the frictional force per unit mass with velocity (solid line) and the downward component of the driving force per unit mass $g \sin \theta$ (dashed line). In the inset, we have plotted the measured velocities (dots) together with the interpolation curve (Legendre polynomials) used in the computations. On the same plot, we have drawn the velocity variations as if the avalanche were in a purely Coulomb regime (dashed line): assuming that the frictional force is in the Coulomb form $F=f m g \cos \theta$, where $f$ is the bulk friction coefficient, we numerically solved the equation of motion (Eq. (52), in which $F_{\mathrm{t}} / m$ is replaced with the expression of $F$ above). As shown in Fig. 27, in the early phases (between points A and B), the frictional force gently decreased with increasing velocity and was slightly lower than the gravity acceleration $g \sin \theta$. Because of the small difference between $g \sin \theta$ and $F / m$, the avalanche accelerated less vigorously than an avalanche in an inertial regime. At instant $\mathrm{B}$, the avalanche reached its maximum velocity $(24 \mathrm{~m} / \mathrm{s})$. At this point, the frictional force started exceeding the gravitational force and the avalanche decelerated monotonically. Obviously, the frictional force did depend on the avalanche velocity, as shown in Fig. 4 , but this dependence remained slight since between $\mathrm{B}$ and $\mathrm{C}$ we have: $F / m \propto u^{0.1 \pm 0.05}$. Thus, as a first approximation, the frictional force can be considered constant between instants A 


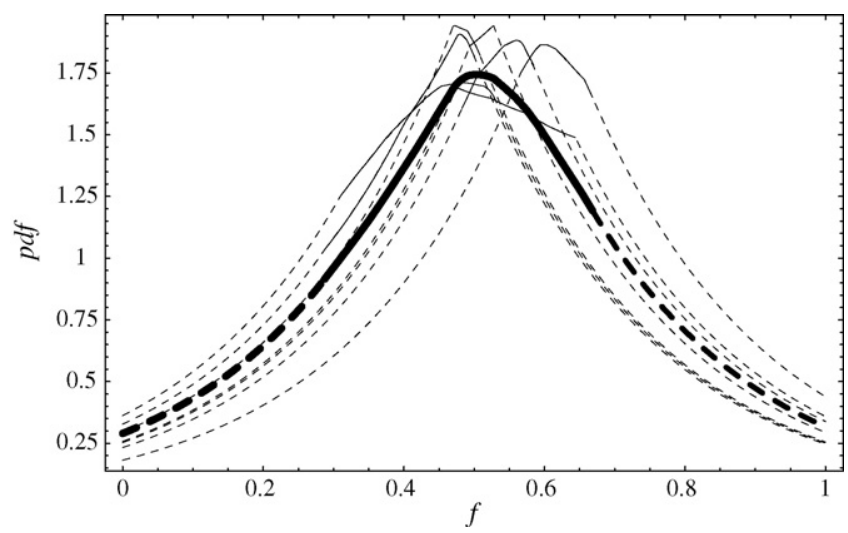

Fig. 28. Empirical probability distribution functions (pdf) of the $173 f$ values collected from the seven paths. The thick line represents the distribution function of the total sample, whereas the thin lines are related to individual paths. Each curve has been split into three parts: the central part (solid line) corresponds to the range of computed $\mu$ values, while the end parts have been extrapolated. After [8].

and C: $F / m=5 \pm 1.3 \mathrm{~m} / \mathrm{s}^{2}$. As shown in the inset of Fig. 27 , the computed velocities obtained by assuming a purely Coulombic regime (dashed curve) compare well with the data: like the recorded values, the computed velocities exhibit an asymmetric U-shaped form, while the relative deviation between the two curves is less than $20 \%$.

For a few events, the bulk frictional force exhibits a dependence on the mean velocity, but no clear trend in the $f(u)$ dependence was found [12]. An interesting property of this simple Coulomb block model is that knowing the run-out distance (point of furthest reach) of an avalanche makes it possible to infer the $f$ value. Since in different alpine regions, avalanche events have been recorded over a long time period at different sites, we can deduce the statistical properties of the $f$ distribution at different places. If the bulk friction coefficient $f$ were a true physical parameter, its statistical properties should not vary with space. Ancey thus conducted a statistical analysis on $f$ values by selecting 173 avalanche data collected from seven sites in France. These sites are known to produce large avalanches and their activity has been followed up since the beginning of the 20th century. Fig. 28 shows the probability distribution of $f$ for each site together with the entire sample. Although the curves are close and similar, they are not statistically identical. This means that the probability distribution function of $f$ is not uniquely determined and depends on other parameters such as snow properties, site configuration, etc. Within this approach, the Coulomb model successfully captures the flow features, but its friction parameter is not a true physical parameter. This, however, should not negate the interest of the Coulomb model because, given the number of approximations needed to derive (52) and (53), the statistical deviance may originate from crude assumptions.

\section{Conclusion}

In this review paper, we have shown various aspects of Coulomb plasticity and viscoplasticity. Although the physical mechanisms on the particle scale are quite similar on numerous points, the rheological properties differ significantly on the bulk scale in the continuum-mechanics description. The key differences lie mainly in the two-phase nature of the bulk and the role of normal stress in the shear-stress generation.

For idealized suspensions of equal-size particles within a Newtonian fluid, microstructural analysis together with dimensional arguments help clarify the physical origins of plasticity and the different forms of plastic behavior. On the whole, this understanding remains qualitative and, although the theoretical predictions are often in agreement with experimental data, full and quantitative agreement is far from complete. In particular, recent experiments have substantiated the notion of timedependent yield stress/surface. Note that in soil mechanics, the nonuniqueness of the yield surface and its history-dependence (hardening/softening) has long been recognized [78,234]. Laboratory experiments carried out on model suspensions have shown that post-yielding behavior is usually properly characterized by either a Coulomb-like or a viscoplastic model depending on the material properties and flow features. Phenomenological laws (e.g., Herschel-Bulkley, Coulomb) successfully capture the salient rheological properties for flow conditions that do not depart significantly from steady, simple-shear flow conditions. Not much experimental work has been accomplished so far on unsteady and three-dimensional flows. In this respect, it is worth noting that to date, as far as I am aware, no rheological determination of the yield surface has been carried out in the rheology of concentrated suspensions.

Contrasting with model suspensions, natural suspensions exhibit a diversity of grain sizes and types, which makes the use of concepts drawn from model suspensions trickier and, perhaps, more deceptive. When the bulk is well sorted with a net separation between the fine and coarse fractions, it usually exhibits viscoplastic properties [218,219]. For poorly sorted materials, there is a nearly continuous size distribution, which gives rise to a wide range of characteristic times in the rheological response of the bulk to a sudden variation in the stress state. This time-dependence of the rheological properties is exacerbated by unsteady processes such as particle sedimentation, cluster formation, and pore-pressure diffusion. According to Iverson, Denlinger, and Major [79,128,157], Coulomb friction and pore pressure diffusion predominate over viscous dissipation, and the Coulomb plastic model provides a correct approximation for describing the time-dependent properties of natural slurries.

The review paper also explores sheet flows of Coulomb or viscoplastic flows. Slow viscoplastic flow can be described using a nonlinear diffusion equation, for which exact or approximate analytical solutions have been provided, shedding light into the features of creeping motion and deposits. Fast motion can be characterized within the framework of flow-depth averaged or Saint-Venant equations. Analytical solutions can also be found for some ideal flow conditions, such as the dambreak problem. To date, most analytical and numerical solutions concern ideal cases, where the rheological properties are simple (i.e., viscoplastic or Coulomb models). Few results have been produced on self-organization (front formation, levee development). A proper treatment of bottom boundary conditions 
(including slipping velocity, mass entrainment/deposition) have also attracted little attention within the geophysics community [33], even though this issue is of prime importance for modeling geophysical flows.

In the last part, we tackle the difficult issue of rheological inference from field data. In addition to parameter fitting and deposit interpretation, we provide the simplest method for deriving rheological properties when the only information available is the front velocity variation along the path. Applications to avalanche data have demonstrated that the Coulomb frictional model captures the salient features of avalanche motion. However, a thorough statistical analysis has shown that the friction coefficient is not a true physical parameter, but depends on the site where the avalanches occurred. This last section emphasizes the importance of the physical reliability of models used in geophysical fluid mechanics, especially when these models are used for engineering purposes.

\section{Acknowledgements}

The work presented here was supported by the Swiss National Science Foundation under grant number 200021-105193/1 and specific funds provided by EPFL (vice-présidence à la recherche). The work presented in this paper was also partially supported by the National Competence Center in Research on Mobile Information and Communication Systems (NCCRMICS), a center supported by the Swiss National Science Foundation under grant number 5005-67322. The author thanks Dr. Richard M. Iverson for providing authorization of reproducing Fig. 20 and the referees for thorough and detailed remarks.

\section{References}

[1] M.J. Adams, Plasticity in concentrated particulate suspensions, in: D Bideau, J. Dodds (Eds.), Physics of Granular Media, Nova Science Publishers, New York, 1991, pp. 365-378.

[2] M.J. Adams, I. Aydin, B.J. Briscoe, S.K. Sinha, A finite element analysis of the squeeze flow of an elasto-viscoplastic paste material, J. NonNewtonian Fluid Mech. 71 (1997) 41-57.

[3] P.M. Adler, H. Brenner, Spatially periodic suspensions of convex particles in linear shear flows, Int. J. Multiphase Flow 11 (1985) 361-385.

[4] W.J. Ammann, A new Swiss test-site for avalanche experiments in the Vallée de la sionne, Cold Regions Sci. Technol. 30 (1999) 3-11.

[5] C. Ancey, Snow avalanches, in: N.J. Balmforth, A. Provenzale (Eds.), Geomorphological Fluid Mechanics: Selected Topics in Geological and Geomorphological Fluid Mechanics, Springer, Berlin, 2001, pp. 319-338.

[6] C. Ancey, Role of lubricated contacts in concentrated polydisperse suspensions, J. Rheol. 45 (2001) 1421-1439.

[7] C. Ancey, Solving the Couette inverse problem by using a waveletvaguelette decomposition, J. Rheol. 49 (2005a) 441-460.

[8] C. Ancey, Monte Carlo calibration of avalanches described as Coulomb fluid flows, Phil. Trans. Roy. Soc. Lond. A 363 (2005b) 1529-1550.

[9] C. Ancey, P. Coussot, Transition from frictional to viscous regime for granular suspensions, C.R. Acad. Sci. Paris Sér. I 327 (1999) 515-522.

[10] C. Ancey, P. Evesque, Frictional-collisional regime for granular suspension flows down an inclined channel, Phys. Rev. E 62 (2000) 8349-8360.

[11] C. Ancey, H. Jorrot, Yield stress for particle suspensions within a clay dispersion, J. Rheol. 45 (2001) 297-319.

[12] C. Ancey, M. Meunier, Estimating bulk rheological properties of flowing snow avalanches from field data, J. Geophys. Res. 109 (2004) F01004.
[13] C. Ancey, P. Coussot, P. Evesque, Examination of the possibility of a fluid-mechanics treatment for dense granular flows, Mech. CohesiveFrict. Mater. 1 (1996) 385-403.

[14] C. Ancey, P. Coussot, P. Evesque, A theoretical framework for very concentrated granular suspensions in a steady simple shear flow, J. Rheol. 43 (1999) 1673-1699.

[15] J.A. Aragon, Granular-fluid chute flow: experimental and numerical observations, J. Hydraul. Eng. 121 (1995) 355-364.

[16] M. Arattano, W.Z. Savage, Modelling of debris flows as kinematic waves, Bull. Int. Assoc. Eng. Geol. 49 (1994) 3-13.

[17] A. Armanini, H. Capart, L. Fraccarollo, M. Larcher, Rheological stratification in experimental free-surface flows of granular-liquid mixtures, J. Fluid Mech. 532 (2005) 269-319.

[18] G. Astarita, The engineering reality of the yield stress, J. Rheol. 34 (1990) 275-277.

[19] R.A. Bagnold, Experiments on a gravity-free dispersion of large solid spheres in a Newtonian fluid under shear, Proc. Roy. Soc. Lond. 225 (1954) 49-63.

[20] N.J. Balmforth, R.V. Craster, A consistent thin-layer theory for Bingham plastics, J. Non-Newtonian Fluid Mech. 84 (1999) 65-81.

[21] N.J. Balmforth, R.R. Kerswell, Granular collapse in two dimensions, J. Fluid Mech. 538 (2005) 399-428.

[22] N.J. Balmforth, A. Provenzale (Eds.), Geomorphological Fluid Mechanics: Selected Topics in Geological and Geomorphological Fluid Mechanics, Springer, Berlin, 2001.

[23] N.J. Balmforth, R.V. Craster, R. Sassi, Shallow viscoplastic flow on an inclined plane, J. Fluid Mech. 470 (2002) 1-29.

[24] N.J. Balmforth, R.V. Craster, R. Sassi, Dynamics of cooling viscoplastic domes, J. Fluid Mech. 499 (2004) 149-182.

[25] M. Barbolini, U. Gruber, C.J. Keylock, M. Naaim, F. Savi, Application of statistical and hydraulic-continuum dense-snow avalanche models to five European sites, Cold Regions Sci. Technol. 31 (2000) 133-149.

[26] E. Bardou, C. Ancey, C. Bonnard, L. Vulliet, Classification of debrisflow deposits for hazard assessment in alpine areas, in: C.L. Chen, D. Rickenmann (Eds.), Debris Flow Mechanics and Mitigation Conference, Mills Press, Davos, 2003, pp. 799-808.

[27] H.A. Barnes, Shear-thickening ("dilatancy") in suspensions of nonaggregating solid particles dispersed in Newtonian liquids, J. Rheol. 33 (1989) 329-366.

[28] H.A. Barnes, Thixotropy-a review, J. Non-Newtonian Fluid Mech. 70 (1997) 1-33.

[29] H.A. Barnes, The yield stress - a review or ' $\pi \alpha \nu \tau \alpha \rho \in \iota$ ' - everything flows? J. Non-Newtonian Fluid Mech. 81 (1999) 213-217.

[30] H.A. Barnes, Q.D. Nguyen, Rotating vane rheometry - A review, J. NonNewtonian Fluid Mech. 98 (2001) 1-14.

[31] H.A. Barnes, K. Walters, The yield stress myth? Rheol. Acta 24 (1985) 324-326.

[32] P. Bartelt, B. Salm, U. Gruber, Calculating dense-snow avalanche runout using a Voellmy-fluid model with active/passive longitudinal straining, J. Glaciol. 45 (1999) 242-254.

[33] P. Bartelt, O. Buser, M.A. Kern, Dissipated work, stability and the internal flow structure of granular snow avalanches, J. Glaciol. 51 (2005) 1-14.

[34] G.K. Batchelor, The stress system in a suspension of free-force particles, J. Fluid Mech. 41 (1970) 545-570.

[35] G.K. Batchelor, Transport properties of two-phase materials with random structure, Annu. Rev. Fluid Mech. 6 (1974) 227-255.

[36] G.K. Batchelor, The effect of Brownian motion on the bulk stress in a suspension of spherical particles, J. Fluid Mech. 83 (1977) 97-117.

[37] G.K. Batchelor, Brownian diffusion with hydrodynamic interaction, J. Fluid Mech. 74 (1977) 1-29.

[38] G.K. Batchelor, J.T. Green, The determination of the bulk stress in a suspension of spherical particles to order $c^{2}$, J. Fluid Mech. 56 (1972) $401-427$.

[39] J.-C. Baudez, P. Coussot, Rheology of aging, concentrated, polymeric suspensions: applications to pasty sewage sludges, J. Rheol. 45 (2001) 1123-1140.

[40] A. Berker, W.E. Van Arsdale, Phenomenological models of viscoplastic, thixotropic, and granular materials, Rheol. Acta 31 (1992) 119-138. 
[41] C.L.A. Berli, D. Quemada, Rheological modeling of microgel suspensions involving solid-liquid transition, Langmuir 16 (2000) 7968-7974.

[42] M. Berti, R. Genevois, A. Simoni, P.R. Tecca, Field observations of a debris flow event in the Dolomites, Geomorphology 29 (1999) 265-274.

[43] E.C. Bingham, Fluidity and Plasticity, McGraw-Hill, New York, 1922.

[44] R.B. Bird, G.C. Dai, B.J. Yarusso, The rheology and flow of viscoplastic materials, Rev. Chem. Eng. 1 (1983) 1-70.

[45] F. Bouchut, A. Mangeney-Castelnau, B. Perthame, J.-P. Vilotte, A new model of Saint Venant and Savage-Hutter type for gravity driven shallow flows, C.R. Acad. Sci. Paris Sér. I 336 (2003) 531-536.

[46] J.F. Brady, The rheological behavior of concentrated colloidal dispersions, J. Chem. Phys. 99 (1993) 567-581.

[47] J.F. Brady, G. Bossis, Stokesian dynamics, Annu. Rev. Fluid Mech. 20 (1988) 111-157.

[48] M. Brocca, Z.P. Bažant, Microplane constitutive model and metal plasticity, Appl. Mech. Rev. 53 (2000) 265-281.

[49] P. Brunn, H. Asoud, Analysis of shear rheometry of yield stress materials and apparent yield stress materials, Rheol. Acta 41 (2002) 524-531.

[50] O. Buser, H. Frutiger, Observed maximum run-out distance of snow avalanches and determination of the friction coefficients $\mu$ and $\xi$, J. Glaciol. 26 (1980) 121-130.

[51] D. Caillerie, Évolution quasi-statique d'un milieu granulaire, loi incrémentale par homogénéisation, in: C. Petit, G. Pijaudier-Cabot, J.M. Reynouard (Eds.), Des géomatériaux aux ouvrages, expérimentations et modélisations, Hermès, Paris, 1995, p. 445.

[52] B. Cambou, P. Dubujet, F. Emeriault, F. Sidoroff, Homogenization for granular materials, Eur. J. Mech. A 14 (1995) 255-276.

[53] B. Cambou, M. Chaze, F. Dedecker, Change of scale in granular materials, Eur. J. Mech. A 19 (6) (2000) 999-1014.

[54] M.E. Cates, J.P. Wittmer, J.-P. Bouchaud, P. Claudin, Jamming, force chains, and fragile matter, Phys. Rev. Lett. 81 (1998) 1841-1844.

[55] C.-L. Chen, Comprehensive review of debris flow modeling concepts in Japan, Rev. Eng. Geol. 7 (1987) 13-29.

[56] C.-L. Chen, C.-H. Ling, Rheological equations in asymptotic regimes of granular flow, J. Eng. Mech. 124 (1998) 301-310.

[57] C.L. Chen, General solutions for viscoplastic debris flow, J. Hydraul. Eng. 114 (1988) 259-282.

[58] D.C.-H. Cheng, Characterisation of thixotropy revisited, Rheol. Acta 42 (2003) 372-382.

[59] D.C.-H. Cheng, Yield stress: a time-dependent property and how to measure it, Rheol. Acta 25 (1986) 542-554.

[60] J. Christoffersen, M.M. Mehrabadi, S. Nemat-Nasser, A micromechanical description of granular behavior, J. Appl. Mech. 48 (1981) 339-344.

[61] V. Chugunov, J.M.N.T. Gray, K. Hutter, Group theoretic methods and similarity solutions of the Savage-Hutter equations, in: K. Hutter, N. Kirchener (Eds.), Dynamics Response of Granular and Porous Materials under Large and Catastrophic Deformations, Springer, Berlin, 2003, pp. 251-261.

[62] S.M. Contreras, T.R.H. Davies, Coarse-grained debris-flows: hysteresis and time-dependent rheology, J. Hydraul. Eng. 126 (2000) 938-941.

[63] C.A. Coulomb, Sur une application des règles de maxima \& minima à quelques problèmes de statique relatifs à 1'architecture, Mémoires de Mathématique et de Physique, Académie Royale de Sciences, Imprimerie Royale, Paris, 1773, pp. 343-382.

[64] P. Coussot, Steady, laminar, flow of concentrated mud dispensions in open channel, J. Hydraul. Res. 32 (1994) 535-559.

[65] P. Coussot, Mudflow Rheology and Dynamics, Balkema, Rotterdam, 1997.

[66] P. Coussot, C. Ancey, Rheophysical classification of concentrated suspensions and granular pastes, Phys. Rev. E 59 (1999) 4445-4457.

[67] P. Coussot, J.M. Piau, A large-scale field coaxial cylinder rheometer for the study of the rheology of natural coarse suspensions, J. Rheol. 39 (1995) 105-124.

[68] P. Coussot, S. Proust, Slow, unconfined spreading of a mudflow, J. Geophys. Res. B101 (1996) 25217-25229.

[69] P. Coussot, S. Proust, C. Ancey, Rheological interpretation of deposits of yield stress fluids, J. Non-Newtonian Fluid Mech. 66 (1996) 55-70.
[70] P. Coussot, D. Laigle, M. Arratano, A. Deganutti, L. Marchi, Direct determination of rheological characteristics of debris flow, J. Hydraul. Eng. 124 (1998) 865-868.

[71] P. Coussot, Q.D. Nguyen, H.T. Huynh, D. Bonn, Viscosity bifurcation in thixotropic, yielding fluids, J. Rheol. 46 (2002) 573-590.

[72] P. Coussot, Q.D. Nguyen, H.T. Huynh, D. Bonn, Avalanche behavior in yield stress fluids, Phys. Rev. Lett. 88 (2002) 175501.

[73] P. Coussot, J.S. Raynaud, C. Ancey, Combined MRI-Rheometry determination of the behavior of mud suspensions, in: C.L. Chen, D. Rickenmann (Eds.), Debris Flow Mechanics and Mitigation Conference, Mills Press, Davos, 2003, pp. 291-301.

[74] P. Coussot, N. Roussel, S. Jarny, H. Chanson, Continuous or catastrophic solid-liquid transition in jammed systems, Phys. Fluids 17 (2005) 011704

[75] S. Dartevelle, Numerical modeling of geophysical granular flows. 1. A comprehensive approach to granular rheologies and geophysical multiphase flows, Geochem. Geophys. Geosyst. 5 (2004) 2003GC000636.

[76] F. Darve (Ed.), Geomaterials: Constitutive equations and Modeling, Elsevier, London, 1989.

[77] T.R.H. Davies, Large debris flow: a macro-viscous phenomenon, Acta Mech. 63 (1986) 161-178.

[78] R.O. Davis, A.P.S. Sevladurai, Plasticity and Geomechanics, Cambridge University Press, Cambridge, 2002.

[79] R.P. Denlinger, R.M. Iverson, Granular avalanches across irregular threedimensional terrain. 1. Theory and computation, J. Geophys. Res. 109 (2004) F01014.

[80] R.P. Denlinger, R.M. Iverson, Flow of variably fluidized granular masses across three-dimensional terrain. 2. Numerical predictions and experimental tests, J. Geophys. Res. 106 (2001) 553-566.

[81] J.D. Dent, T.E. Lang, Modelling of snow flow, J. Glaciol. 26 (1980) 131140 .

[82] J.D. Dent, T.E. Lang, Experiments on the mechanics of flowing snow, Cold Regions Sci. Technol. 5 (1982) 243-248.

[83] J.D. Dent, T.E. Lang, A biviscous modified Bingham model of snow avalanche motion, Ann. Glaciol. 4 (1983) 42-46.

[84] R.H. Dressler, Unsteady non-linear waves in sloping channels, Proc. Roy. Soc. Lond. A 247 (1958) 186-198.

[85] D.A. Drew, S.L. Passman, Theory of Multicomponent Fluids, Springer, New York, 1999.

[86] D.C. Drucker, W. Prager, Soil mechanics and plastic analysis or limit design, Quarter. J. Appl. Math. 10 (1952) 157-165.

[87] S. Egashira, T. Itoh, H. Takeushi, Transition mechanics of debris flows over rigid bed to over erodible bed, Phys. Chem. Earth B 26 (2001) 169174.

[88] A. Elverhøi, D. Issler, F.V. De Blasio, T. Ilstad, C.B. Harbitz, P. Gauer, Emerging insights into the dynamics of submarine debris flows, Nat. Hazard Earth. Sys. Sci. 5 (2004) 633-648.

[89] P. Evesque, C. Stefani, Relationship between dilatancy, stresses and plastic dissipation in a granular material with rigid grains, J. Phys. II 1 (1991) 1337-1347.

[90] G. Félix, N. Thomas, Relation between dry granular flow regimes and morphology of deposits: formation of levées in pyroclastic deposits, Earth Planet. Sci. Lett. 221 (2004) 197-213.

[91] S.M. Fielding, P. Sollich, M.E. Cates, Aging and rheology in soft materials, J. Rheol. 44 (2000) 323-369.

[92] N.A. Frankel, A. Acrivos, On the viscosity of a concentrated suspension of solid spheres, Chem. Eng. Sci. 22 (1967) 847-853.

[93] GDR-MIDI, On dense granular flows, Eur. Phys. J. E 14 (2004) 341-365.

[94] T.A. Ghezzehei, D. Or, Rheological properties of wet soils and clays under steady and oscillatory stresses, Soil Sci. Soc. Am. J. 65 (2001) 624-637.

[95] J.D. Goddard, An elastohydrodynamic theory for the rheology of concentrated suspensions of deformable particles, J. Non-Newtonian Fluid Mech. 2 (1977) 169-189.

[96] J.D. Goddard, Dissipative materials as models of thixotropy and plasticity, J. Non-Newtonian Fluid Mech. 14 (1984) 141-160.

[97] J.D. Goddard, A.K. Didwania, Computations of dilatancy and yield surfaces for assemblies of rigid frictional spheres, Quarter. J. Mech. App. Math. 51 (1998) 15-43. 
[98] I. Goldhirsch, Rapid granular flows, Annu. Rev. Fluid Mech. 35 (2003) 267-293.

[99] P. Gondret, P. Petit, Viscosité des suspensions désordonnées et ordonnées de sphères solides: expériences et modèles, C. R. Acad. Sci. Paris Sér. IIb 321 (1995) 25-31.

[100] J. Gratton, C. Vigo, Self-similar gravity currents with variable inflow revisited: plane currents, J. Fluid Mech. 258 (1994) 77-104.

[101] J.M.N.T. Gray, M. Wieland, K. Hutter, Gravity-driven free surface flow of granular avalanches over complex basal topography, Proc. Roy. Soc. Lond. A 455 (1998) 1841-1874.

[102] R. Greve, T. Koch, K. Hutter, Unconfined flow of granular avalanches along a partly curved surface. I. Theory, Proc. Roy. Soc. Lond. A 445 (1994) 399-413.

[103] R.W. Griffiths, The dynamics of lava flows, Annu. Rev. Fluid Mech. 32 (2000) 477-518.

[104] H. Gubler, Dense-flow avalanches, a discussion of experimental results and basic processes, in: L. Buisson (Ed.), International Workshop on rapid gravitational mass movements, Cemagref, Grenoble, 1993, pp. 126-127.

[105] M.A. Hampton, H.J. Lee, J. Locat, Submarine landslides, Rev. Geophys. 34 (1996) 33-59.

[106] J.P. Harnett, R.Y.Z. Hu, The yield stress—an engineering reality, J. Rheol. 33 (1989) 671-679.

[107] D.A. Head, A. Ajdari, M.E. Cates, Jamming, hysteresis, and oscillation in scalar models for shear thickening, Phys. Rev. E 65061509 (2001).

[108] L. Heymann, S. Peukert, N. Aksel, On the solid-liquid transition of concentrated suspensions in transient shear flow, Rheol. Acta 41 (2002) 307315.

[109] P.Y. Hicher, A. Rahma, Micro-macro correlations for granular media. Application to the modelling of sands, Eur. J. Mech. A 13 (6) (1994) 763-781.

[110] R. Hill, The Mathematical Theory of Plasticity, Oxford University Press, Oxford, 1950.

[111] E.J. Hinch, An averaged-equation approach to particle interactions in a fluid suspension, J. Fluid Mech. 83 (1977) 695-720.

[112] R.L. Hoffman, Discontinuous and dilatant viscosity behavior in concentrated suspensions. I. Observations of a flow instability, Trans. Soc. Rheol 16 (1972) 155-173.

[113] R.L. Hoffman, Explanations for the cause of shear thickening in concentrated colloidal suspensions, J. Rheol. 42 (1998) 111-123.

[114] A.J. Hogg, D. Pritchard, The effects of hydraulic resistance on dam-break and other shallow inertial flows, J. Fluid Mech. 501 (2004) 179-212.

[115] K. Hohenemser, W. Prager, Über die Ansätze der Mechanik der isotroper Kontinua, Z. Angew. Math. Mech. 12 (1932) 216-226.

[116] X. Huang, M.H. Garcìa, A perturbation solution for Bingham-plastic mudflows, J. Hydraulic Eng. 123 (1997) 986-994.

[117] X. Huang, M.H. Garcìa, A Herschel-Bulkley model for mud flow down a slope, J. Fluid Mech. 374 (1998) 305-333.

[118] O. Hungr, R.R. Morgenstern, Experiments on the flow behaviour of granular materials at high velocity in an open channel, Géotechnique 34 (1984) 405-413.

[119] B. Hunt, Newtonian fluid mechanics treatment of debris flows and avalanches, J. Hydraul. Eng. 120 (1994) 1350-1363.

[120] H.E. Huppert, The intrusion of fluid mechanics into geology, J. Fluid Mech. 173 (1986) 557-594.

[121] M. Hürlimann, D. Rickenmann, C. Graf, Field and monitoring data of debris-flow events in the Swiss Alps, Can. Geotech. J. 40 (2003) 161175.

[122] D.M. Husband, N. Aksel, W. Geissle, The existence of static yield stresses in suspensions containing noncolloidal particles, J. Rheol. 37 (1993) 215235.

[123] K. Hutter, K. Jöhnk, Continuum Methods of Physical Modeling, Springer, Berlin, 2004.

[124] K. Hutter, T. Koch, C. Plüss, S.B. Savage, The dynamics of avalanches of granular materials from initiation to runout. Part II: Experiments, Acta Mech. 109 (1995) 127-165.

[125] K. Hutter, B. Svendsen, D. Rickenmann, Debris flow modeling: a review, Continuum Mech. Thermodyn. 8 (1996) 1-35.
[126] R.M. Iverson, How should mathematical models of geomorphic processus to be judged? in: P.R. Wilcock, R.M. Iverson (Eds.), Prediction in Geomorphology, American Geophysical Union, Washington, DC, 2003, pp. 83-94.

[127] R.M. Iverson, The debris-flow rheology myth, in: C.L. Chen, D. Rickenmann (Eds.), Debris flow Mechanics and Mitigation Conference, Mills Press, Davos, 2003, pp. 303-314.

[128] R.M. Iverson, Debris-flow mechanics, in: M. Jakob, O. Hungr (Eds.), Debris-flow Hazards and Related Phenomena, Springer, Berlin, 2005, pp. 105-134.

[129] R.M. Iverson, The physics of debris flows, Rev. Geophys. 35 (1997) $245-$ 296.

[130] R.M. Iverson, R.P. Denlinger, Flow of variably fluidized granular masses across three-dimensional terrain. 1. Coulomb mixture theory, J. Geophys. Res. 106 (2001) 537-552.

[131] R.M. Iverson, R.G. LaHusen, Friction in debris flows: Inferences from large-scale flume experiments, in: American Society of Civil Engineers (Ed.), Hydraulic Engineering '93, vol. 2, 1993.

[132] A.M. Johnson, Physical Process in Geology, Freeman, Cooper \& Co, San Francisco, 1970

[133] A.M. Johnson, J.R. Rodine, Debris flow, in: D. Brunsden, D.B. Prior (Eds.), Slope Instability, John Wiley \& Sons, Chichester, 1984, pp. 257362.

[134] A.I. Jomha, A. Merrington, L.V. Woodcock, H.A. Barnes, A. Lips, Recent developments in dense suspension rheology, Powder Technol. 65 (1990) 343-370.

[135] D.A.R. Jones, B. Leary, D.V. Boger, The rheology of a concentrated colloidal suspension of hard sphere, J. Colloid. Interface Sci. 147 (1991) 479-495.

[136] P. Jop, Y. Forterre, O. Pouliquen, Crucial role of side walls for granular surface flows: consequences for the rheology, J. Fluid Mech. 541 (2005) 167-192.

[137] C. Josserand, P.-Y. Lagrée, D. Lhuillier, Stationary shear flows of dense granular materials: a tentative continuum modelling, Eur. Phys. J. E 14 (2004) 127-135

[138] P.C. Kapur, P.J. Scales, B.V. Boger, T.W. Healy, A theoretical framework for the yield stress of suspensions loaded with size distributed particles, AIChE J. 43 (1997) 1171-1179.

[139] K.V. Karelsky, V.V. Papkov, A.S. Petrosyan, D.V. Tsygankov, Particular solutions of shallow-water equations over a non-flat surface, Phys. Lett. A 271 (2000) 341-348.

[140] J.B. Keller, Shallow-water theory for arbitrary slopes of the bottom, J. Fluid Mech. 489 (2003) 345-348.

[141] M.A. Kern, F. Tiefenbacher, J.N. McElwaine, The rheology of snow in large chute flows, Cold Regions Sci. Technol. 39 (2004) 181-192.

[142] R.R. Kerswell, Dam break with Coulomb friction: a model for granular slumping? Phys. Fluids 17 (2005) 057101.

[143] D.L. Koch, R.J. Hill, Inertial effects in suspension and porous-media flows, Annu. Rev. Fluid Mech. 33 (2001) 619-647.

[144] H.K. Kytömaa, D. Prasad, Transition from quasi-static to rate dependent shearing of concentrated suspensions, in: C. Thornton (Ed.), Powders \& Grains, Balkema, Birmingham, 1993, pp. 281-287.

[145] R.J. LeVeque, Finite Volume Methods for Hyperbolic Problems, Cambridge University Press, Cambridge, 2002.

[146] D. Lhuillier, Ensemble averaging in slightly non-uniform suspensions, Eur. J. Mech. B 6 (1992) 649-661.

[147] M.J. Lighthill, G.B. Whitham, On kinematic waves. I. Flood movement in long rivers, Proc. Roy. Soc. Lond. A 229 (1955) 281-316.

[148] G.G. Lipscomb, M.M. Denn, Flow of Bingham fluids in complex geometries, J. Non-Newtonian Fluid Mech. 14 (1984) 337-346.

[149] K.F. Liu, C.C. Mei, Approximate equations for the slow spreading of a thin sheet of Bingham plastic fluid, Phys. Fluids A2 (1990) 30-36.

[150] K.F. Liu, C.C. Mei, Slow spreading of a sheet of Bingham fluid on an inclined plane, J. Fluid Mech. 207 (1990) 505-529.

[151] K.F. Liu, C.C. Mei, Roll waves on a layer of a muddy fluid flowing down a gentle slope-A Bingham model, Phys. Fluids 6 (1990) 2577-2590.

[152] D. Lootens, H. Van Damme, P. Hébraud, Giant stress fluctuations at the jamming transition, Phys. Rev. Lett. 90 (2003) 178301. 
[153] B. Loret, Simple potential elastoplasticity, in: F. Darve (Ed.), Geomaterials: Constitutive Equations and Modeling, Elsevier, London, 1989, pp. 149-186.

[154] T.S. Lundgren, Slow flow through stationary random beads and suspensions of spheres, J. Fluid Mech. 51 (1972) 273-299.

[155] J.J. Major, Gravity-driven consolidation of granular slurriesimplications for debris-flow deposition and deposit characteristics, J. Sediment. Res. 70 (2000) 64-83.

[156] J.J. Major, Experimental studies of deposition of debris flows: process, characteristics of deposits, and effects of pore-fluid pressure, Ph.D., Washington, 1996.

[157] J.J. Major, Depositional processes in large-scale debris-flow experiments, J. Geol. 105 (1997) 345-366.

[158] J.J. Major, T.C. Pierson, Debris flow rheology: experimental analysis of fine-grained slurries, Water Resour. Res. 28 (1992) 841-857.

[159] J.J. Major, R.M. Iverson, D.F. McTigue, S. Macias, B.K. Fiedorowicz, Geotechnical properties of debris-flow sediments and slurries, in: C.-L. Chen (Ed.), Debris Flow Mechanics and Mitigation Conference, 1997, pp. 249-259.

[160] G. Mandl, R. Fernandez-Luque, Fully developed plastic shear flow of granular materials, Géotechnique 20 (1970) 277-307.

[161] A. Mangeney, P. Heinrich, R. Roche, Analytical solution for testing debris avalanche numerical models, Pure Appl. Geophys. 157 (2000) 10811096.

[162] M. Marchioro, M. Tanksley, W. Wang, A. Prosperetti, Flow of spatially non-uniform suspensions. Part II: Systematic derivation of closure relations, Int. J. Multiphase Flow 27 (2001) 237-276.

[163] M. Marquez, A. Robben, B.P. Grady, I. Robb, Viscosity and yield stress reduction in non-colloidal concentrated suspensions by surface modification with polymers and surfactants and/or nanoparticle addition, J. Colloid. Interface Sci. 295 (2006) 374-387.

[164] R. Martino, Experimental analysis on the rheological properties of a debris-flow deposit, in: C.L. Chen, D. Rickenmann (Eds.), Debris Flow Mechanics and Mitigation Conference, Mills Press, Davos, 2003, pp. 363373.

[165] G.P. Matson, A.J. Hogg, Two-dimensional dam break flows of HerschelBulkley fluids: the approach to the arrested state. J. Non-Newtonian Fluid Mech. 142 (2007) 79-94.

[166] B.W. McArdell, P. Bartelt, The role of granular agitation and pore water pressure in the basal shearing of debris flows. submitted to Geophys. Rev. Lett., 2006.

[167] B.W. McArdell, B. Zanuttigh, A. Lamberti, D. Rickenmann, Systematic comparison of debris flow laws at the Illgraben torrent, Switzerland, in: C.L. Chen (Ed.), Debris flow Mechanics and Mitigation Conference, Mills Press, Davos, 2003, pp. 647-657.

[168] D.M. McClung, Superelevation of flowing avalanches around curved channel bends, J. Geophys. Res. 106 (B8) (2001) 16489-16498.

[169] D.L. McDowell, Modeling and experiments in plasticity, Int. J. Solids Struct. 37 (2000) 293-309.

[170] C.C. Mei, M. Yuhi, Slow flow of a Bingham fluid in a shallow channel of finite width, J. Fluid Mech. 431 (2001) 135-159.

[171] C.C. Mei, K.F. Liu, M. Yuhi, Mud flows-slow and fast, in: N.J. Balmforth, A. Provenzale (Eds.), Geomorphological Fluid Mechanics: selected topics in geological and geomorphological fluid mechanics, Springer, Berlin, 2001, pp. 548-577.

[172] M. Meunier, C. Ancey, J.-M. Taillandier, Fitting avalanche-dynamics models with documented with documented events from the Col du Lautaret site (France) using the conceptual approach, Cold Regions Sci. Technol. 39 (2004) 55-66.

[173] O. Mohr, Welche Umstände bedingen die Elastizitäsgrenze und den Bruch eines Materials? Zeit. Vereines Deutsch. Ing. 44 (1900) 1-12.

[174] P. Mougin, Les avalanches en Savoie, volume IV. Ministère de l'Agriculture, Direction Générale des Eaux et Forêts, Service des Grandes Forces Hydrauliques, Paris, 1922.

[175] D.M. Mueth, Measurements of particle dynamics in slow, dense granular Couette flow, Phys. Rev. E 67 (2003) 011304.

[176] F. Nicot, F. Darve, A multi-scale approach to granular materials, Mech. Mater. 37 (2005) 980-1006.
[177] K. Nishimura, K. Kogusi, M. Nakagawa, Experiments on ice-sphere flows along an inclined chute, Mech. Mater. 16 (1993) 205-209.

[178] K. Nishimura, S. Keller, J. McElwaine, Y. Nohguchi, Ping-pong ball avalanche at a ski jump, Granul. Matter 1 (1998) 51-56.

[179] H.F. Norem, F. Irgens, B. Schieldrop, A continuum model for calculating snow avalanche velocities, in: H. Gubler, B. Salm (Eds.), Avalanche Formation, Movement and Effects, IAHS, Davos, Wallingford, Oxfordshire, UK, 1986, pp. 363-379.

[180] J.S. O’Brien, P.Y. Julien, Laboratory analysis of mudflow properties, J. Hydraul. Eng. 114 (1988) 877-887.

[181] C.S. O’Hern, S.A. Langer, A.J. Liu, S.R. Nagel, Force distributions near jamming and glass transitions, Phys. Rev. Lett. 86 (2001) 0031.

[182] F. Oka, Validity and limits of the effective stress concept in geomechanics, Mech. Cohesive-Frict. Mater. 1 (1996) 219-234.

[183] J.G. Oldroyd, A rational formulation of the equations of plastic flow for a Bingham solid, Proc. Camb. Phil. Soc. 43 (1947) 100-105.

[184] J.G. Oldroyd, On the formulation of rheological equations of state, Proc. Roy. Soc. Lond. A 200 (1950) 523-541.

[185] D.I. Osmond, R.W. Griffiths, The static shape of yield strength fluids slowly emplaced on slopes, J. Geophys. Res. B106 (2001) 16241-16250.

[186] T.C. Papanastasiou, Flows of materials with yield, J. Rheol. 31 (1987) 385-404.

[187] F. Parsi, F. Gadala-Maria, Fore-and-aft asymmetry in a concentrated suspension of solid spheres, J. Rheol. 31 (1987) 725-732.

[188] J.D. Parsons, K.X. Whipple, A. Simoni, Experimental study of the grain-flow, fluid-mud transition in debris flows, J. Geol. 109 (2001) 427-447.

[189] M. Pastor, M. Quecedo, E. González, M.I. Herreros, J.A. Fernández, P. Mira, Simple approximation to bottom friction for Bingham fluid depth integrated models, J. Hydraul. Eng. 130 (2004) 149-155.

[190] C.J. Phillips, T.R.H. Davies, Determining rheological parameters of debris flow material, Geomorphology 4 (1991) 101-110.

[191] J.-M. Piau, Flow of a yield stress fluid in a long domain. Application to flow on an inclined plane, J. Rheol. 40 (1996) 711-723.

[192] D. Picard, A. Adjari, F. Lequeux, L. Bocquet, Slow flows of yield stress fluids: complex spatiotemporal behavior within a simple elastoplastic model, Phys. Rev. E 71 (2005) 010501.

[193] A.A. Potanin, W.B. Russel, Fractal model of consolidation of weakly aggregated colloidal dispersions, Phys. Rev. E 53 (1996) 3702-3709.

[194] A.A. Potanin, R. De Rooi, D. Van den Ende, J. Mellema, Microrheological modeling of weakly aggregated dispersions, J. Chem. Phys. 102 (1995) 5845-5853.

[195] O. Pouliquen, Y. Forterre, Friction law for dense granular flow: application to the motion of a mass down a rough inclined plane, J. Fluid Mech. 453 (2002) 133-151.

[196] W. Prager, Introduction to Mechanics of Continua, Ginn and Company, Boston, 1961.

[197] D. Prasad, H.K. Kytömaa, Particle stress and viscous compaction during shear of dense suspensions, Int. J. Multiphase Flow 21 (1995) 775-785.

[198] A. Prosperetti, The average stress in incompressible disperse flow, Int. J. Multiphase Flow 30 (2004) 1011-1036.

[199] S.P. Pudasaini, K. Hutter, Rapid shear flows of dry granular masses down curved and twisted channels, J. Fluid Mech. 495 (2003) 193-208.

[200] F. Radjai, M. Jean, J.J. Moreau, S. Roux, Force distributions in dense two-dimensional granular systems, Phys. Rev. Lett. 77 (1996) 274-277.

[201] K.R. Rajagopal, A.R. Srinivasa, On the thermomechanics of materials that have multiple natural configurations. Part I: Viscoelasticity and classical plasticity, Z. Angew. Math. Phys. 55 (2004) 861-893.

[202] J. Rajchenbach, Granular flows, Adv. Phys. 49 (2000) 229-256.

[203] A. Remaître, J.-P. Malet, O. Maquaire, C. Ancey, J. Locat, Flow behaviour and runout modelling of a complex debris flow in a clay-shale basin, Earth Surf. Proc. Landforms 30 (2005) 479-488.

[204] K.H. Roscoe, The influence of strains in soil mechanics, Géotechnique 20 (1970) 129-170.

[205] W.B. Russel, Review of the role of colloidal forces in the rheology of suspensions, J. Rheol. 24 (1980) 287-317.

[206] W.B. Russel, D.A. Saville, W.R. Schowalter, Colloidal Dispersions, Cambridge University Press, Cambridge, 1995. 
[207] S.E.H. Sakimoto, T.K.P. Gregg, Channeled flow: analytical solutions, laboratory experiments, and applications to lava flows, J. Geophys. Res., B 106 (2001) 8629-8644.

[208] J. Salençon, An introduction to the yield design theory and its application to soil mechanics, Eur. J. Mech. A 5 (1990) 477-500.

[209] M. Sambridge, K. Mosegaard, Monte Carlo methods in geophysical inverse problems, Rev. Geophys. 40 (2002) 1-29.

[210] S.B. Savage, Granular flows down rough inclined-review and extension, in: J.T. Jenkins, M. Satake (Eds.), US/Japan Seminar on New Models and Constitutive Relations in the Mechanics of Granular Materials, Elseviers Science Publishers, Ithaca, Amsterdam, 1982, pp. 261-282.

[211] S.B. Savage, Flow of granular materials, in: P. Germain, J.-M. Piau, D. Caillerie (Eds.), Theoretical and Applied Mechanics, Elsevier, Amsterdam, 1989, pp. 241-266.

[212] S.B. Savage, K. Hutter, The motion of a finite mass of granular material down a rough incline, J. Fluid Mech. 199 (1989) 177-215.

[213] S.B. Savage, R.M. Iverson, Surge dynamics coupled to pore-pressure evolution in debris flows, in: C.L. Chen, D. Rickenmann (Eds.), Debris Flow Mechanics and Mitigation Conference, Mills Press, Davos, 2003, pp. 503-514.

[214] S.B. Savage, Y. Nohguchi, Similarity solutions for avalanches of granular materials down curved bed, Acta Mech. 75 (1988) 153-174.

[215] P.J. Scales, P.C. Kapur, S.B. Johnson, T.W. Healy, The shear yield stress of partially flocculated colloidal suspensions, AIChE J. 44 (1998) $538-544$.

[216] M. Schatzmann, P.F. Fischer, G.R. Bezzola, Rheological behavior of fine and large particle suspensions, J. Hydraul. Eng. 129 (2003) 796-803.

[217] M.A. Schofield, C.P. Wroth, Critical State of Soil Mechanics, McGrawHill, Lond., 1968.

[218] M.Z. Sengun, R.F. Probstein, Bimodal model of slurry viscosity with applications to coal slurries. Part 1. Theory and experiment, Rheol. Acta 28 (1989) 382-393.

[219] M.Z. Sengun, R.F. Probstein, Bimodal model of slurry viscosity with applications to coal slurries. Part 2. High shear limit behaviour, Rheol. Acta 28 (1989) 394-401.

[220] M.C. Shen, R.E. Meyer, Climb of a bore on a beach, J. Fluid Mech. 16 (1963) 125-133.

[221] S. Siavoshi, A. Kudrolli, Failure of a granular step, Phys. Rev. E 71 (2005) 051302.

[222] L.E. Silbert, D. Ertas, G.S. Grest, T.C. Hasley, D. Levine, Analogies between granular jamming and the liquid-glass transition, Phys. Rev. E 65 (2002) 051307.

[223] A. Siviglia, A. Cantelli, Effect of bottom curvature on mudflow dynamics: theory and experiments, Water Resour. Res. 41 (2005) W11423.

[224] P. Sollich, Rheological constitutive equation for a model of soft glassy materials, Phys. Rev. E 58 (1998) 738-759.

[225] P. Sollich, F. Lequeux, P. Hébraud, M.E. Cates, Rheology of soft glassy materials, Phys. Rev. Lett. 78 (1997) 2020-2023.

[226] J.J. Stickel, R.L. Powell, Fluid mechanics and rheology of dense suspensions, Annu. Rev. Fluid Mech. 37 (2005) 129-149.
[227] T. Takahashi, Debris flow, Annu. Rev. Fluid Mech. 13 (1981) 57-77.

[228] T. Takahashi, Debris flow. IAHR Monograph, Balkema, Rotterdam, 1991.

[229] J.H. Trowbridge, Instability of concentrated free surface flows, J. Geophys. Res., C 92 (1987) 9523-9530.

[230] C. Truesdell, Six Lectures on Modern Natural Philosophy, Springer Verlag, New York, 1966.

[231] J.-C. Tsai, W. Losert, G.A. Voth, Two-dimensional granular Poiseuille flow on an incline: multiple dynamical regimes, Phys. Rev. E 65 (2001) 011306.

[232] M. Tubino, S. Lanzoni, Rheology of debris flows: experimental observations and modelling problems, Excerpta 7 (1993) 201-235.

[233] J.C. van der Werff, V.F. de Kruif, Hard-sphere colloidal dispersions: the scaling of rheological properties with particle size, volume fraction, and shear rate, J. Rheol. 33 (1989) 421-454.

[234] I. Vardoulakis, J. Sulem, Bifurcation Analysis in Geomechanics, Blackie Academic \& Professional, Glasgow, 1995

[235] C. Völtz, M. Nitschke, L. Heymann, I. Rehberg, Thixotropy in macroscopic suspensions of spheres, Phys. Rev. E 65 (2002) 051402.

[236] G.J. Weir, The asymptotic behaviour of simple kinematic waves of finite volume, Proc. Roy. Soc. Lond. A 387 (1983) 459-467.

[237] K.X. Whipple, Open-Channel flow of Bingham fluids: application in debris-flow research, J. Geol. 105 (1997) 243-262.

[238] C.R. Wildemuth, M.C. Williams, A new interpretation of viscosity and yield stress in dense slurries: coal and other irregular particles, Rheol. Acta 24 (1985) 75-91.

[239] S.K. Wilson, B.R. Duffy, A.B. Ross, On the gravity-driven draining of a rivulet of a viscoplastic material down a slowly varying substrate, Phys. Fluids 14 (2002) 555-571.

[240] T.A. Witten, P. Pincus, Structured Fluids, Oxford University Press, Oxford, 2004

[241] Y.L. Yeow, W.C. Ko, P.P.P. Tang, Solving the inverse problem of Couette viscometry by Tikhonov regularization, J. Rheol. 44 (2000) 1335-1351.

[242] B. Zanuttigh, A. Lamberti, Analysis of debris flow wave development with one-dimensional shallow-water equations, J. Hydraul. Eng. 130 (2004) 293-304.

[243] I.E. Zarraga, D.A. Hill, D.T. Leighton, The characterization of the total stress of concentrated suspensions of noncolloidal spheres in Newtonian fluids, J. Rheol. 44 (2000) 185-221.

[244] D.Z. Zhang, Evolution of enduring contacts and stress relaxation in a dense granular medium, Phys. Rev. E 71 (2005) 041303.

[245] D.Z. Zhang, A. Prosperetti, Momentum and energy equations for disperse two-phase flows and their closure for dilute suspensions, Int. J. Multiphase Flow 23 (1997) 425-453.

[246] D.Z.Zhang, R.M. Rauenzahn, Stress relaxation in dense and slow granular flows, J. Rheol. 44 (2000) 1019-1041.

[247] Z. Zhou, M.J. Solomon, P.J. Scales, D.V. Boger, The yield stress of concentrated flocculated suspensions of size distributed particles, J. Rheol. 43 (1999) 651-671.

[248] H. Zhu, Y.D. Kim, D. De Kee, Non-Newtonian fluids with a yield stress, J. Non-Newtonian Fluid Mech. 129 (2005) 177-181. 